\title{
Linking microgel particle properties to filtration behavior through microscopic observations
}

Izabella Bouhid de Aguiar 



\section{Propositions}

1. The starting point for membrane selection should be the size of soft particles under process conditions not their size at "rest".

(this thesis)

2. Water intrusion is an essential prerequisite in cleaning processes related to removal of compressed microgel packings.

(this thesis)

3. The word micro should only be used to refer to objects that are in the micrometer size range.

4. In quantitative image analysis, good thresholding is key.

5. Labelling foreigners according to the circumstances of their arrival does not help integration.

6. In the era of smart machines, people should be encouraged to go back to basics and use their brains.

Propositions belonging to the thesis, entitled

'Linking microgel particle properties to filtration behavior through microscopic observations'

Izabella Bouhid de Aguiar

Wageningen, 5 June 2019 

Linking microgel particle properties to

filtration behavior through microscopic observations 


\section{Thesis Committee}

\section{Promotor}

Prof. Dr C.G.P.H. Schroën

Personal chair at the Laboratory of Food Process Engineering

Wageningen University \& Research

\section{Co-promotors}

Dr M. Meireles

Research Director, Laboratoire de Génie Chimique

Université de Toulouse III - Paul Sabatier, France

Dr A. Bouchoux

Researcher, Laboratoire d'Ingénierie des Systèmes Biologiques et des Procédés Université de Toulouse - INSA, France

\section{Other members}

Prof. Dr A. van der Wal, Wageningen University \& Research

Prof. Dr D.C. Nijmeijer, Eindhoven University of Technology

Dr P. Menut, Montpellier SupAgro, France

Dr P. Aimar, Université de Toulouse III - Paul Sabatier, France

This research was conducted under the auspices of Graduate School VLAG (Advanced studies in Food Technology, Agrobiotechnology, Nutrition and Health Sciences). 


\title{
Linking microgel particle properties to filtration behavior through microscopic observations
}

\author{
Izabella Bouhid de Aguiar
}

\section{Thesis}

submitted in fulfilment of the requirements for the degree of doctor at Wageningen University by the authority of the Rector Magnificus, Prof. Dr A.P.J. Mol, in the presence of the

Thesis Committee appointed by the Academic Board to be defended in public on Wednesday June $5^{\text {th }}, 2019$ at 11 a.m. in the Aula. 
Izabella Bouhid de Aguiar

Linking microgel particle properties to filtration behavior through microscopic observations

137 pages

$\mathrm{PhD}$ thesis, Wageningen University, Wageningen, the Netherlands (2019)

With references, with summaries in English, French and Dutch

ISBN: 978-94-6343-438-6

DOI: https://doi.org/10.18174/471626 


\section{Contents}

Chapter 1 Introduction 1

Chapter 2 Compressive resistance of granular-scale microgels: From loose to dense packing

Chapter 3 Deswelling and deformation of microgels in concentrated packings

Chapter 4 Microfluidic model systems used to emulate processes occurring during soft particle filtration

Conformational changes influence clogging behavior of

Chapter 5 micrometer-sized microgels in idealized multiple constrictions

Chapter 6 Discussion

Summary

Résumé

Samenvatting

List of publications

Acknowledgements 

Introduction 


\section{General concepts studied in this thesis}

This thesis investigates the behavior of soft particles during filtration. Before focusing on filtration, we first discuss a number of general concepts such as hardness/softness, and collective behavior of particles. Next, the general principles of filtration are introduced, from which the innovative aspects of this thesis follow, including the description of the contents of this thesis.

\section{Soft versus hard}

The difference between soft and hard is one of the first things we learn when discovering the world in our early years, by falling down (and getting up again). And maybe for this reason, we do not really pay so much attention to how present these two concepts are in our lives. Nobody questions the fact that billiard balls are hard and do not change regardless of how they are used. On the other hand, slime and squishy toys that very popular with children these days, can be easily deformed and/or compressed, exemplifying very well the concept of softness.

In general, soft materials present a different behavior in comparison with hard materials [1,2]. Soft materials can deform, be squeezed, and even shrink, depending on their composition and characteristics what makes them different from hard particles [3-8]. Soft materials deform when their shape deviates from their original shape [9-11], as is the case of slime balls. Deswelling or squeezing happens when a material such as a balloon loses volume [12,13]; both effects can happen simultaneously in squishy toys (Figure 1).

\begin{tabular}{|l|c|c|c|c|}
\hline & Hard & \multicolumn{3}{|c|}{ Soft } \\
\hline Deform & $\mathbf{X}$ & $\checkmark$ & $\checkmark$ & $\mathbf{X}$ \\
\hline Deswell & $\mathbf{X}$ & $\checkmark$ & $\mathbf{X}$ & $\checkmark$ \\
\hline & Billiards & Squishies & Slime balls & Balloons \\
\hline
\end{tabular}

(13) 13
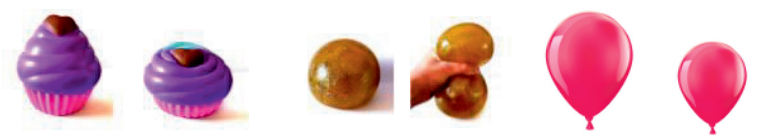

Figure 1. Differences between hard and soft materials.

\section{Particulate systems}

Particles are everywhere and particulate systems are part of our daily lives more than we realize, ranging from food ingredients such as rice and soy beans in silos to the children's ball pit at the playground. These examples sound very different from each 
other. However, the one thing these systems have in common is the fact that they are composed of individual particles but utilized in collectivity. What we can observe is that the properties of the individual components (particle size, sphericity) together with the properties of the collective system (packing density, concentration) will determine collective system behavior [14,15].

Collective systems of soft particles can, amongst others, be found in food products and ingredients, proteins and pharmaceutical products [16]. As the name already suggests they are not rigid, and have the ability to modify their conformation according to the surrounding environment (and processing conditions as will be explained later). For this reason, system packing density, concentration, and particle properties will be a function of external parameters, leading to soft particles displaying a much richer behavior as their hard counterparts [3-8].

At a microscopic scale, soft particles are present in many industrial processes such as dairy [17], pharmaceutical $[18,19]$ and waste water treatment $[20,21]$. For example, in milk, casein micelles are present as suspended components and are very important structure elements in milk $[8,22,23]$ and during further processing thereof ranging e.g. from milk filtration to cheese fabrication [24]. In the pharmaceutical field, carrier particles for drug delivery such as microgels are prepared and have to be further processed $[18,19]$. In waste water treatment, sludge flocs form and also need subsequent processing $[20,21]$. These are all soft particles and due to their specific behaviors, many issues arise during processing, such as formation of irreversible deposits, change of particle properties and consequent product losses are some of the issues that soft particles are linked to $[4,5,25]$.

A process that is especially prone to changing behavior of soft particles, and is used in all of the industries mentioned above is filtration [26-28]; therefore, we investigated various aspects related to filtration and took this as a focal point for our research.

\section{Filtration}

Filtration is a well-known unit operation that is widely used to separate components from a stream with the use of a filtration medium that can vary in composition, ranging from fabrics to ceramics to membranes. In general, filtration is used to recover, purify or fractionate industrial streams [29-31].

One type of filtration process is membrane filtration. In membrane filtration, the pores of the filter medium are microscopic or even smaller, and for that reason driving forces such as differences in pressure, temperature and solute concentration 
are required to achieve separation [32]. Some membrane processes such as microfiltration are based on the size exclusion principle, which dictates that in a membrane process, the great majority of particles bigger than the membrane molecular weight cut off will be retained while smaller particles are able to pass through the membrane pores [33,34]. This is in general true for individual hard particles, since they do not change their conformation during the filtration process, but once present at high concentration, also the collective behavior of the particles will influence the separation that is achieved. For example, through the formation of a cake layer (see Figure 2), the separation process is influenced $[35,36]$ as discussed later.

The size exclusion principle has to be considered more carefully for soft particles, due to their deformability and deswelling properties [37-39]. Depending on the process, soft particles need to be purified or removed if they are undesired [29-31]. Processing should be adjusted to, ideally, include the special behaviors of soft particles to reach effective separation despite the final objective of the process. In practice, this is not taken into account yet.

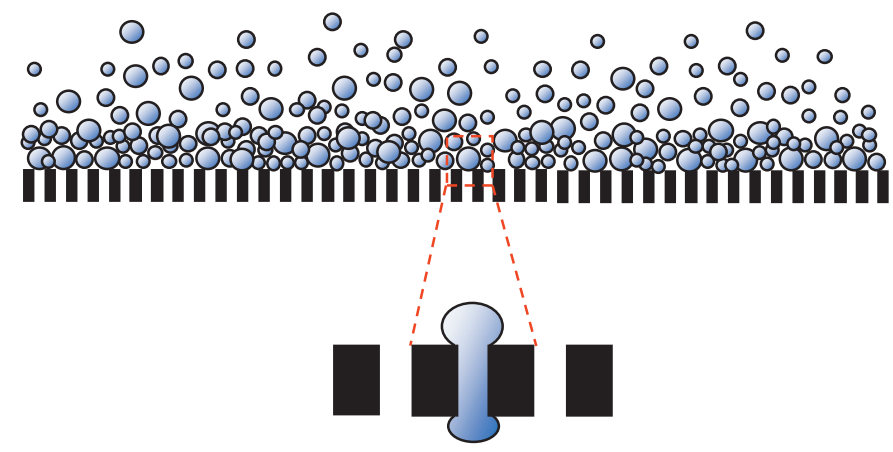

Figure 2. Cake layer formation and pore clogging by a soft particle.

Solute rejection is a common way to classify performance of membrane processes based on size exclusion. It determines the percentage of solute that is retained by the membrane. The Ferry-Renkin equation is one example of equations that have been proposed to describe membrane rejection $(\mathbb{R})$ (Equation (1) [40].

$$
\mathbb{R}=\left[1-2\left(1-\frac{a}{r}\right)^{2}+\left(1-\frac{a}{r}\right)^{4}\right] \times 100 \%
$$

where $r$ is the radius of ideal cylindrical pores, and $a$ is the solute radius. 
As can be seen, particle size in relation to pore size (ratio $a / r$ ) is strongly incorporated in the equation. But what if the solute particle size changes during processing (as illustrated in Figure 2)? For this reason, understanding the behavior and properties of soft particles is of key importance, as discussed in chapters 2-6.

Fouling and cake formation are inherent phenomena of membrane filtration and decrease process efficiency [41]. Fouling happens when the membrane pores are reversibly or irreversibly blocked by a particle present in the feed stream; in this thesis we call this pore clogging. Cake formation is the name given to the accumulation of particles on top of the filtration medium (Figure 2) [42,43].

Cake formation is a consequence of a phenomena called concentration polarization $[44,45]$ (or of pore clogging if retention changes because of it). The fact that components/particles are retained by the membrane induces concentration gradients between the membrane and the bulk feed. Mathematical descriptions of concentration polarization phenomena generally are based on such concentration term [40].

$$
J_{i}=k_{m}\left(c_{i_{o}}-c_{i_{p}}\right)
$$

Where $J_{i}$ is the flux of component $i, k_{m}$ is the mass transfer coefficient and $\left(c_{i_{o}}-c_{i_{p}}\right)$ is the overall concentration difference across the boundary layer. For simplicity we do not take the concentration difference across the membrane into account, although this obviously also may play a role.

But what if the concentration of the solute constantly changes not only due to further accumulation of particles on the membrane, but also due to particle shape changes happening locally due to the compressible and deformable nature of soft particles? This makes behavior prediction and process optimization very challenging.

\section{Thesis outline}

In this thesis (Figure 3), we are digging deep into the understanding of soft particle behavior that is relevant for pore clogging and cake formation. Throughout the thesis we will use microgels that can be easily produced on lab scale and have tunable properties [46,47], which makes them ultimately suitable as model particles for fundamental research $[48,49]$. The average size of microgels can vary from nanometer to micrometer range [50-53]. A great variety of studies focusses on colloidal microgels that also exhibit colloidal interactions [54-58]. Here we use microgels for which colloidal interactions are negligible $[4,59]$ in order to be able to 
fully focus on deformability of the particles, in some cases in combination with highly idealized microfluidic model membranes.

We start our research by studying the behavior of soft particles on macroscale, using static and isotropic conditions (Figure 3 left). In this situation, the particles are experiencing the same forces coming from every direction [60], and for this we use the osmotic stress technique [61] to observe the behavior of microgels packings; highly concentrated microgel suspensions with individual microgels in a jammed state $[14,15]$. The microgel particles are subjected to isotropic forces since they are surrounded by neighboring microgels in a three-dimensional way [62].

Isotropic/Static

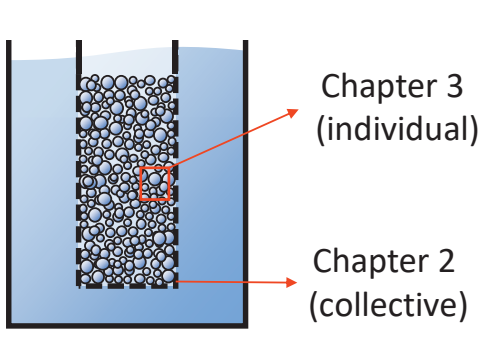

\section{Anisotropic/Dynamic}

Chapter 4

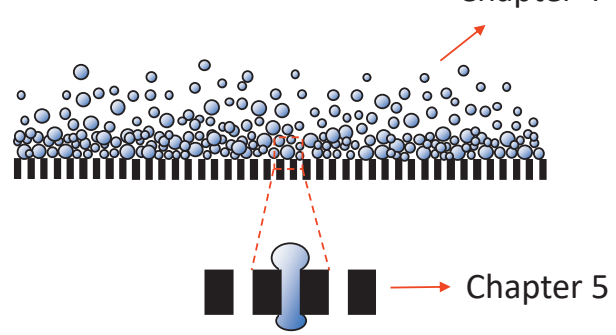

Figure 3. Schematic representation of the thesis outline.

The microgel packings are used to simulate particle behavior in cake layers. In Chapter 2, we focus on the particle collective behavior in static conditions. We describe the behavior of microgels in packings in terms of osmotic pressure [63]. We also make use of well-known models to support our observations. In Chapter 3, we look into individual particle behavior also in static conditions. We make use of fluorescence microscopy and image analysis to quantitatively determine individual particle deformation and deswelling in packings.

From Chapter 4 onwards (Figure 3 right), we focus on micrometer scale observations of particles in anisotropic dynamic systems. In these systems, we can observe the behavior of soft particles moving through pores and under varying applied forces. In our study we use microfluidic centrifugation [64] and filtration devices. In Chapter 4 we investigate collective particle behavior. We use a microcentrifuge coupled to an optical microscope to observe the deposition of microgels under centrifugal force [65]. The use of centrifugation allows us to apply high pressures to the system. We also assess the reversibility of the deposits according to the applied force. 
Centrifugation can be used to observe the behavior of microgel packings under force but not under a constant flow; for this, other microfluidic tools are needed as reported in chapters 4 and 5 . Microfluidic devices have been widely used for the insitu observation of micrometer-sized particles in motion [66-68], and can be tailor made for applications such as microfluidic filtration $[66,68]$. In this thesis, an array of channels is used to simulate a filtration medium containing different pore geometries, and used to observe the filtration process. In Chapter 4, we use microfluidic devices to investigate the collective behavior in dynamic systems, and focus on cake layer formation as function of time and applied pressure. We also assess cake reversibility. The suspension of microgels in the microfluidic devices are also subjected to convective forces and in Chapter 5 , we focus on individual particles going through pore constrictions (individual behavior under dynamic conditions). We correlate the observations with particle and system properties such as particle size and applied pressure.

Finally, in Chapter 6, we discuss our main findings and their implications in real life situations and processes. We found that microgels use mainly two mechanisms to accommodate pressure: deswelling and deformation that mostly occur simultaneously to different degrees according to process parameters and possibly microgel properties. We obtained information on how the individual particle behavior will determine the structure and properties of collective systems as for example in cake/deposit layers and gathered more information on individual particles going through constrictions due to size adjustment. These findings are expected to be of great relevance for filtration processes.

Still in Chapter 6, we discuss topics that came up during our observations but were not subject of extensive research in this thesis. These topics, such as microgel reswelling kinetics, are described as perspectives for future research. 


\section{References}

[1] B. Sierra-Martin, J.A. Frederick, Y. Laporte, G. Markou, J.J. Lietor-Santos, A. Fernandez-Nieves, Determination of the bulk modulus of microgel particles. Colloid Polym. Sci. 289 (2011) 721-728.

[2] J. Mattsson, H.M. Wyss, A. Fernandez-Nieves, K. Miyazaki, Z. Hu, D.R. Reichmann, D.A. Weitz, Soft colloids make strong glasses, Nature 462 (2009) 83-86.

[3] A. Bouchoux, P. Qu, P. Bacchin, G. Gésan-Guiziou, A general approach for predicting the filtration of soft and permeable colloids: the milk example, Langmuir 30 (2014) 22-34.

[4] O. Nir, T. Trieu, S. Bannwarth, M. Wessling, Microfiltration of deformable microgels, Soft Matter. 12 (2016) 6512-6517.

[5] W.M. Lu, K.L. Tung, C.H. Pan, K.J. Hwang, Crossflow microfiltration of mono-dispersed deformable particle suspension, J. Membr. Sci. 198 (2002) 225243.

[6] K.J. Hwang, C.L. Hsueh, Dynamic analysis of cake properties in microfiltration of soft colloids, J. Membr. Sci. 214 (2003) 259-273.

[7] K. Horigome, D. Suzuki, Drying mechanism of poly(N-isopropylacrylamide) microgel dispersions, Langmuir 28 (2012) 12962-12970.

[8] C. Sadek, L. Pauchard, P. Schuck, Y. Fallourd, N. Pradeau, C. Le Floch-Fouéré, R. Jeantet, Mechanical properties of milk protein skin layers after drying: understanding the mechanisms of particle formation from whey protein isolate and native phosphocaseinate, Food Hydrocoll. 48 (2015) 8-16.

[9] S.P. Meeker, R.T. Bonnecaze, M. Cloitre, Slip and flow in soft particle pastes, Phys. Rev. Lett. 92 (2004) 198302-1.

[10] L. Nyström, R. Álvarez-Asencio, G. Frenning, B.R. Saunders, M.W. Rutland, M. Malmsten, Electrostatic swelling transitions in surface-bound microgels, ACS Appl. Mater. Inter. 8, (2016) 27129-27139.

[11] J. Maldonado-Valderrama, T. del Castillo-Santaella, I. Adroher-Benítez, A. Moncho-Jordá, A. Martín-Molina, Thermoresponsive microgels at the airwater interface: the impact of the swelling state on interfacial conformation, Soft Matter 8 (2016) 6416.

[12] C. Pellet, M. Cloitre, The glass and jamming transitions of soft polyelectrolyte microgel suspensions, Soft Matter 12 (2016) 3710-3720.

[13] J.J. Lietor-Santos, B. Sierra-Martin, R. Vavrin, Z. Hu, U. Gasser, A. FernandezNieves, Deswelling microgel particles using hydrostatic pressure, Macromolecules 42 (2009) 6225-6230.

[14] A. Stradner, J.J. Crassous, K. van Gruijthuijsen, M. Obiols-Rabasa, P. Schurtenberger, P.S. Mohanty, R. Schweins, S. Nöjd, Interpenetration of polymeric microgels at ultrahigh densities, Sci. Rep. 7 (2017) 1487.

[15] G.M. Conley, P. Aebischer, S. Nöjd, P. Schurtenberger, F. Scheffold, Jamming and overpacking fuzzy microgels: deformation, interpenetration, and compression, Sci. Adv. 3 (2017) e1700969.

[16] C.N. Likos, Soft matter with soft particles, Soft Matter 2 (2006) 478-498. 
[17] C.G. de Kruif, T. Huppertz, V.S. Urban, A.V. Petukhov, Adv. Colloid Interface Sci. 171 (2012) 36-52.

[18] B.R. Saunders, N. Laajam, E. Daly, S. Teow, X. Hu, R. Stepto, Microgels: from responsive polymer colloids to biomaterials, Adv. Colloid Interface Sci. 147148 (2009) 251-262.

[19] J.K. Oh, R. Drumright, D.J. Siegwart, K. Matyjaszewski, The development of microgels/nanogels for drug delivery applications, Prog. Polym. Sci. 33 (2008) 448-477.

[20] Y. Jeong, Y. Kim, Y. Jin, S. Hong, C. Park, Comparison of filtration and treatment performance between polymeric and ceramic membranes in anaerobic membrane bioreactor treatment of domestic wastewater, Sep. Purif. Technol. 199 (2018) 182-188.

[21] L. Wang, W. Liang, W. Chen, W. Zhang, J. Mo, K. Liang, B. Tang, Y. Zheng, F. Jiang, Integrated aerobic granular sludge and membrane process for enabling municipal wastewater treatment and reuse water production, Chem. Eng. J. 337 (2018) 300-311.

[22] A. Bouchoux, B. Debbou, G. Gesan-Guiziou, M.H. Famelart, J.L. Doublier, B. Cabane, Rheology and phase behavior of dense casein micelle dispersions, J. Chem. Phys. 131 (2009) 165106-165111.

[23] A. Bouchoux, P.E. Cayemitte, J. Jardin, G. Gésan-Guiziou, B. Cabane, Casein micelle dispersions under osmotic stress, Biophys. J. 96 (2009) 693-706.

[24] D.G. Dalgleish, M. Corredig, The structure of the casein micelle of milk and its changes during processing, Annu. Rev. Food Sci. Technol. 3 (2012) 449-467.

[25] W. Guo, H.-H. Ngo, J. Li, A mini-review on membrane fouling, Bioresource Technol. 122 (2012) 27-34.

[26] S.F. Hansen, B. Petrat-Melin, J.T. Rasmussen, L.B. Larsen, M.S. Ostenfeld, L. Wiking, Placing pasteurisation before or after microfiltration impacts the protein composition of milk fat globule membrane material, Int. Dairy J. 81 (2018) 35-41.

[27] R. Roa, D. Menne, J. Riest, P. Buzatu, E.K. Zholkovskiy, J.K. G. Dhont, M. Wessling, G. Nägele, Ultrafiltration of charge-stabilized dispersions at low salinity, Soft Matter 12 (2016) 4638-4653.

[28] L. Wang, W. Liang, W. Chen, W. Zhang, J. Mo, K. Liang, B. Tang, Y, Zheng, $\mathrm{F}$, Jiang, Integrated aerobic granular sludge and membrane process for enabling municipal wastewater treatment and reuse water production, Chem. Eng. J. 337 (2018) 300-311

[29] H.F. Liu, J. Ma, C. Winter, R. Bayer, Recovery and purification process development

for monoclonal antibody production, mAbs 2 (2010) 480-499.

[30] M. Kalyanpur, Downstream processing in the biotechnology industry, Mol. Biotechnol. 22 (2002) 87-98.

[31] T. Burnouf, Modern plasma fractionation, Transfus. Med. Rev. 21 (2007) 101117.

[32] J. Strathmann, Membrane separation process, J. Mem. Sci. 9 (1981) 121-189. 
[33] A.M.C. Van Dinther, C.G.P.H. Schroën, R.M. Boom, Separation process for very concentrated emulsions and suspensions in the food industry, Innov. Food Sci. Emerg. Technol. 18 (2013) 177-182.

[34] K. J. Hwang, Y.C. Chiang, Comparisons of membrane fouling and separation efficiency in protein/polysaccharide cross-flow microfiltration using membranes with different morphologies, Sep. Purif. Technol. 125 (2014) 7482.

[35] E. Iritani, N. Katagiri, Y. Ishikawa, D.-Q. Cao, Cake formation and particle rejection in microfiltration of binary mixtures of particles with two different sizes, Sep. Purif. Technol. 123 (2014) 214-220.

[36] D. Jeison, J.B. van Lier, Cake formation and consolidation: Main factors governing the applicable flux in anaerobic submerged membrane bioreactors (AnSMBR) treating acidified wastewaters, Separation Purif. Technol. 56 (2007) 71-78.

[37] C.W. Hsu, Y.L. Chen, Migration and fractionation of deformable particles in microchannel, J. Chem. Phys. 133 (2010) 034906.

[38] G. Cevc, D. Gebauer, Hydration-driven transport of deformable lipid vesicles through fine pores and the skin barrier, Biophys. J. 84 (2003) 1010-1024.

[39] T.F. Headen, S.M. Clarke, A. Perdigon, G.H. Meeten, J.D. Sherwood, M. Aston, Filtration of deformable emulsion droplets, J. Colloid Interface Sci. 304 (2006) 562-565.

[40] R. Baker, Membrane Technology and Applications, 2nd ed., John Wiley \& Sons, Ltd, Chichester, 2004.

[41] I.M. Griffiths, A. Kumar, P. S. Stewart, A combined network model for membrane fouling, J. Colloid Interface Sci. 432 (2014) 10-18.

[42] H.P. Chu, X.-Y. Li, Membrane fouling in a membrane bioreactor (MBR): sludge cake formation and fouling characteristics, Biotechnol. Bioeng. 90 (2005) 323331.

[43] L. Huang, M.T. Morrissey, Fouling of membranes during microfiltration of surimi wash water: roles of pore blocking and surface cake formation, J. Membr. Sci. 144 (1998) 113-123.

[44] P. Bacchin, D. Si-Hassen, V. Starov, M.J. Clifton, P. Aimar, A unifying model for concentration polarization, gel-layer formation and particle deposition in cross-flow membrane filtration of colloidal suspensions, Chem. Eng. Sci. 57 (2002) 77-91.

[45] V. Chen, A.G. Fane, S. Madaeni, I.G. Wenten, Particle deposition during membrane filtration of colloids: transition between concentration polarization and cake formation, J. Membr. Sci. 125 (1997) 109-122.

[46] M. Destribats, V. Lapeyre, M. Wolfs, E. Sellier, F. Leal-Calderon, V. Ravaine, V. Schmitt, Soft microgels as Pickering emulsion stabilisers: role of particle deformability, Soft Matter 7 (2011) 7689-7698.

[47] C.M. Nolan, L.T. Gelbaum, L.A. Lyon, $1 \mathrm{H}$ NMR investigation of thermally triggered insulin release from poly( $\mathrm{N}$-isopropylacrylamide) microgels, Biomacromolecules 6 (2006) 2918-2922. 
[48] B. Sung, C. Kim, M.H. Kim, Biodegradable colloidal microgels with tunable thermosensitive volume phase transitions for controllable drug delivery, J. Colloid Interf. Sci. 450 (2015) 26-33.

[49] M. Guo, H.M. Wyss, Micromechanics of soft particles, Macromol. Mater. Eng. 296 (2011) 223-229.

[50] F.A. Plamper, W. Richtering, Functional microgels and microgel systems, Acc. Chem. Res. 50 (2017) 131-140.

[51] J.J.F. Sleeboom, P. Voudouris, M.T.J.J. M. Punter, F.J. Aangenendt, D. Florea, P. Van der Schoot, H.M. Wyss, Compression and reswelling of microgel particles after an osmotic shock, Phys. Rev. Lett. 119 (2017) 098001.

[52] P. Menut, S. Seiffert, J. Sprakel, D.A. Weitz, Does size matter? Elasticity of compressed suspensions of colloidal- and granular-scale microgels, Soft Matter. 8 (2012) 156-164.

[53] B.R. Saunders, B. Vincent, Microgel particles as model colloids: theory, properties and applications, Adv. Colloid Interface Sci. 80 (1999) 1-25.

[54] A. Fernández-Nieves, A. Fernández-Barbero, B. Vincent, F.J. de las Nieves, Charge controlled swelling of microgel particles, Macromolecules 33 (2000) 2114-2118.

[55] K. Han, R. Tiwari, T. Heuser, A. Walther, Simple platform method for the synthesis of densely functionalized microgels by modification of active ester latex particles, Macromol. Rapid Comm. 37 (2016) 1323-1330.

[56] M. Pelaez-Fernandez, A. Souslov, L.A. Lyon, P.M. Goldbart, A. FernandezNieves, Impact of single-particle compressibility on the fluid-solid phase transition for ionic microgel suspensions, Phys. Rev. Lett. 114 (2015) 098303.

[57] A. Scotti, U. Gasser, E.S. Herman, M. Pelaez-Fernandez, J. Han, A. Menzel, L.A. Lyon, A. Fernández-Nieves, The role of ions in the self-healing behavior of soft particle suspensions, Proc. Natl. Acad. Sci. 20 (2016) 5576-5581.

[58] A. Scotti, U. Gasser, E.S. Herman, J. Han, A. Menzel, L.A. Lyon, A. FernandezNieves, Phase behavior of binary and polydisperse suspensions of compressible microgels controlled by selective particle deswelling, Phys. Rev. E 96 (2017) 032609 .

[59] K.L. Tung, C.C. Hu, C.J. Chuang, K.J. Hwang, T.Y. Wu, Effects of soft particle deformability and particle/pore size ratio on the blocking mechanism in deadend microfiltration, Chem. Eng. Technol. 33 (2010) 1341-1348

[60] B. Sierra-Martin, J.A. Frederick, Y. Laporte, G. Markou, J.J. Lietor-Santos, A. Fernandez-Nieves, Determination of the bulk modulus of microgel particles. Colloid Polym. Sci. 289 (2011) 721-728.

[61] C. Bonnet-Gonnet, L. Belloni, B. Cabane, Osmotic pressure of latex dispersion, Langmuir 10 (1994) 4012-4021.

[62] I. Bouhid de Aguiar, T. van de Laar, M. Meireles, A. Bouchoux, J. Sprakel, K. Schroën, Deswelling and deformation of microgels in concentrated packings, Sci. Rep. 7 (2017) 10223.

[63] M. Rubinstein, R.H. Colby, A.V. Dobrynin, Dynamics of semidilute polyelectrolyte solutions, Phys. Rev. Lett. 73 (1994) 2776-2779. 
[64] G. Petzold, J. M. Aguilera, Centrifugal freeze concentration, Innov. Food Sci. Emerg. 20 (2013) 253-258

[65] T. Krebs, D. Ershov, C.G.P.H. Schroën, R.M. Boom, Coalescence and compression in centrifuged emulsions studied with in situ optical microscopy, Soft Matter 9 (2013) 4026.

[66] P. Bacchin, A. Marty, P. Duru, M. Meireles, P. Aimar, Colloidal surface interactions and membrane fouling: investigations at pore scale, Adv. Colloid Interface Sci. 164 (2011) 2-11.

[67] H. Di, G.J.O. Martin, D.E. Dunstan, A microfluidic system for studying particle deposition during ultrafiltration, J. Membr. Sci. 532 (2017) 68-75.

[68] R. van Zwieten, T. van de Laar, J. Sprakel, K. Schroën, From cooperative to uncorrelated clogging in cross-flow microfluidic membranes, Sci. Rep. 8 (2018) 5687. 


\section{Compressive resistance of granular-scale microgels: From loose to dense packing}

This chapter has been published as:

Izabella Bouhid de Aguiar, Karin Schroën, Martine Meireles, Antoine Bouchoux Compressive resistance of granular-scale microgels: From loose to dense packing Colloids and Surfaces A, vol 553, p 406-416, 2018 


\section{Abstract}

Microgels are deformable and compressible particles that can be packed to concentrations that exceed the random close packing limit of hard spheres. To reach high packing levels, one has to overcome the resistance to compression of the system. This resistance potentially originates from many different phenomena (thermal agitation effects, surface interactions, microgel deformation, interpenetration, water expulsion) that depend on the microgel properties (size, ionic charge, structure, softness). Here, we investigate granular-scale dextran-based microgels with different native water contents. The resistance to compression of the suspensions is measured through the variation of the osmotic pressure with packing concentration. In parallel, we characterize the structure of the packings in terms of polymer heterogeneity, microgel deformation, and average size using confocal microscopy. We find that all microgel suspensions resist compression in the same manner; however, the mechanisms involved clearly depend on the actual degree of compression. In the loose packing regime, the resistance originates mainly from the resistance of the microgels to their own deformation, with no or negligible deswelling; the osmotic pressure rises abruptly with concentration in analogy to compressed emulsion droplets. In the second and dense packing regime, the microgels necessarily have to expel water to withstand compression. The resistance of the packing is then similar to that of a continuous gel of the same polymer. Importantly, we find that structural macro-voids are still observable in these systems; the presence of which needs to be taken into account when modeling the osmotic resistance. 


\subsection{Introduction}

Microgels are granular- or colloidal- scale particles made of a low-density polymer network swollen by a solvent [1-4]. They are soft and deformable objects that have the ability to change their size and shape in response to their environment, e.g. $\mathrm{pH}$, ionic strength, temperature, concentration. This makes them interesting for a variety of applications such as drug delivery or food formulation for instance [5-7]. They are also model particles that are used for understanding the general behavior of soft objects in various situations. One specific and interesting case is when the particles are highly concentrated and packed against each other. This corresponds to situations encountered in the filtration of milk for instance, and more generally in processes in which filtration, centrifugation or drying operations are used involving deformable and compressible particles [8-13]. The rheological and phase properties of microgel dispersions at increasing concentration are more and more documented [14-18]. The most recent works report a complex phase transition from the fluid to the glassy or solid state; sometimes exhibiting phase coexistence $[19,20]$. This results from the ability of microgels to deswell depending on particle stiffness, ionic environment, size polydispersity and packing concentration [21-23]. The structural properties of the resulting packings, including the way the individual particles deform, organize themselves, sometimes crystallize, and even interpenetrate as a function of concentration, is also a recent matter of interest [24-28]. In particular, whereas crystallization is suppressed for hard colloidal spheres with polydispersity greater than $10 \%$, microgels can overcome this limitation because a small number of large particles can spontaneously deswell to fit in the crystal lattice of smaller microgels $[21,29,30]$. Here we explore another property of microgel packings, which is the resistance to deswelling upon compression of the system. This question has clearly been overlooked over the past few years while it is of crucial importance for understanding and predicting the performances of concentration processes, e.g. drying time or filtration fluxes [8,31-34].

The resistance of a particulate and/or polymeric dispersion/solution to an isotropic compression can be accessed directly by measuring the variation in osmotic pressure with concentration $[35,36]$. The osmotic pressure is the result of all interactions in the system. For colloidal dispersions, it originates from thermal agitation of particles and surface interactions [35]. For polymer solutions, it is given by the entropy of mixing of the polymer segments with the solvent and an additional and often dominating contribution of the polymer counter-ions in the case of polyelectrolytes $[37,38]$. For polymer gels, the mixing and ionic contributions are complemented with a - negative - elastic term that comes from the crosslinks that prevent full reswelling of the structure [39]. The compressive resistance of a single microgel particle similarly 
results from all these contributions. Therefore, osmotic pressure models developed for polymeric gels are now commonly used for explaining the (de)swelling behavior of individual microgels; popular approaches being based on Flory-Rehner theory $[2,40-45]$.

The resistance to compression of a collection of microgels is more complex to analyze. At low concentrations, when the microgels are still separated from each other, surface interactions and thermal agitation often dominate like in the hard sphere dispersion [46-49]. In the specific case of charged, colloidal pNIPAM microgels, these contributions are supplemented by the presence of free counterions in the solution surrounding the particles [19,21,22]. In contrast, at high concentrations, the microgel particles can pack very densely and form a fully homogeneous material that resists compression like a macroscopic polymeric gel [3]. In between these two extreme conditions, the microgels are forced to get into contact with each other but still do not fill all of the available volume and voids are present. The resistance of the packing to compression is then difficult to apprehend as it potentially depends on many different phenomena: thermal agitation, particleparticle interactions, compression of individual microgels (including compression without deformation [24]), deformation (including deformation at constant volume like in emulsion packings [50]), interpenetration [25,26], crystallization [19,20$22,29,30]$, presence of structural heterogeneities. To date, experimental or simulation data on these systems are very much lacking and it is still a challenge to predict and understand what determines their resistance to compression.

Here we examine this question through an experimental study performed with microgels of different origins and stiffness. To simplify the problem, we choose neutral (dextran-based) and granular-scale microgels so that both ionic effects and particle thermal agitation can be safely ignored. Suspensions of microgels are compressed to different degrees and the osmotic pressure of the packings is measured. In parallel, confocal scanning imaging is used to characterize the structure of the packing in terms of polymer heterogeneity and microgel deformation and size.

\subsection{Experimental}

\section{Microgels}

All the microgels that we used are neutral, dextran-based particles (Table 1).

G100-89 and G25-68 microgels are commercial Sephadex particles obtained through crosslinking of dextran polymer by epichlorohydrin [51]. The number after the letter $\mathrm{G}$ is the approximate water content as given by the manufacturer (GE Healthcare 
Life Sciences) in gram of water per gram of dry polymer. MD-66 and MD-61 are methacrylated dextran (dexMA) microgels that we synthesized in our laboratory from dextran T40 using water-in-water emulsion polymerization following the protocol of Stenekes et al. [52,53] (see Appendix A for details about their preparation).

Table 1: Properties of the microgel particles.

\begin{tabular}{|c|c|c|c|c|}
\hline & G100-89 & G25-68 & MD-66 & MD-61 \\
\hline $\begin{array}{l}\text { Native internal water content } \\
\qquad w_{\text {water }}(\% \mathrm{w} / \mathrm{w})\end{array}$ & 89 & 68 & 66 & 61 \\
\hline $\begin{array}{l}\text { Native internal dextran concentration } \\
\qquad C_{i, 0}(\mathrm{~g} / \mathrm{L})\end{array}$ & 114 & 362 & 387 & 460 \\
\hline $\begin{array}{l}\text { Native mean diameter } \\
\qquad d_{0}(\mu \mathrm{m})\end{array}$ & 62.5 & 51.7 & 30.0 & 37.6 \\
\hline $\begin{array}{l}\text { Polydispersity } \\
\qquad 2 \sigma / d_{0}(-)\end{array}$ & 0.40 & 0.18 & 0.20 & 0.20 \\
\hline $\begin{array}{l}\text { Hard-sphere random close packing lim } \\
\text { estimated from Schaertl et al. [54] } \\
\phi_{H S, R C P}(-)\end{array}$ & 0.78 & 0.68 & 0.68 & 0.68 \\
\hline
\end{tabular}

The four microgel particles have different crosslink densities and consequently swell to different degrees when dispersed in water. According to Refs. [45,55], the mesh sizes of the fully swollen microgels vary from $\sim 20 \mathrm{~nm}$ (G100-89) to $\sim 5 \mathrm{~nm}$ (MD61). In Table 1, we provide the values of the corresponding native internal water contents $w_{\text {water }}$ in $\mathrm{g}$ of water per $100 \mathrm{~g}$ total $(\% \mathrm{w} / \mathrm{w})$. These values also appear in the names of the particles after the dash. The water contents were determined using a well-established protocol of Stenekes et al. [55,56]. In brief the concentration of a 2 MDa blue dextran tracer solution is measured after lyophilized microgel particles are added to the solution. The tracer is too large to penetrate the microgel particles when they swell to their equilibrium water content. The increase in tracer concentration in the solution is thus directly related to the quantity of water incorporated in the microgels. In addition to the value of $w_{\text {water }}$, we provide in Table 1 the internal dextran concentration $C_{i, 0}$ of the fully swollen microgels; this characteristic is defined as:

$$
C_{i, 0}=\frac{\left(1-w_{\text {water }}\right)}{\left(1-w_{\text {water }}\right) / d_{\text {dex }}+w_{\text {water }} / d_{\text {water }}}
$$


where $d_{\text {dex }}=1598 \mathrm{~g} / \mathrm{L}[57,58]$ and $d_{\text {water }}=997 \mathrm{~g} / \mathrm{L}$ are the dextran and water densities at $25^{\circ} \mathrm{C}$, respectively.

All the microgels are a few tens of microns in diameter, which allows us to monitor their changes in shape and size in concentrated packings using optical microscopy. Their native size distributions were determined using confocal microscopy with microgel suspensions at low concentration. The microgels are polydisperse with a Gaussian population centered at diameter $d_{0}$ and with a polydispersity $2 \sigma / d_{0}$ ranging from 0.18 to 0.4 (Table $1, \sigma$ is the standard deviation). From the values of polydispersity, we can evaluate the volume fraction at random close packing $\phi_{H S, R C P}$ from the theoretical work of Schaertl et al. on hard-sphere dispersions [54]. This value corresponds to the limit in volume fraction when polydisperse hard spheres are closely packed. It exceeds the 0.64 value for monodisperse spheres because polydisperse systems can pack more efficiently with small particles that fit in the interstices of larger ones. For microgel particles, $\phi_{H S, R C P}$ typically corresponds to the volume fraction above which the microgels necessarily have to deform and/or deswell for the system to reach higher concentrations.

As reported by Flodin, Sephadex microgel particles are homogeneous in polymer and crosslink densities [51], and this is also the case for the dextran microgels that are synthesized through water-in-water emulsion polymerization (see the confocal images in [59] for instance). Therefore, we are far from the core-shell structure of pNIPAM colloidal microgels that have fuzzy shells that can interpenetrate at high concentrations [25,26]. As we will show later, interpenetration effects are most probably very limited - if not absent - in the case of granular scale dextran microgels.

\section{Osmotic stress experiments}

The osmotic stress method was used to compress the microgel suspensions and measure their osmotic pressure. The microgel suspensions are placed in dialysis bags that are in turn immersed in a large volume of a stressing polymer solution of known osmotic pressure. The dialysis bags are chosen so that they respectively retain the microgel particles and the polymer, in the dialysis bag and in the immersion solution. The difference in osmotic pressure, i.e., the difference in water chemical potential, between the two compartments induces an osmotic flow of water from the bag towards the immersion solution. The flow stops when the osmotic pressure in the bag equals the osmotic pressure of the immersion solution. By multiplying the experiments with different concentrations of stressing polymer in the immersion solution, it is possible to compress the microgel suspensions to different levels. The 
resistance to compression of each system is given by the osmotic pressure at equilibrium, i.e. when flow stops.

A polyethylene glycol of $35 \mathrm{kDa}$ molecular weight (PEG35, Sigma-Aldrich) was used as the stressing polymer. The variation of the osmotic pressure of PEG35 solutions as a function of concentration is given in the literature [46,60]. PEG35 concentrations were chosen in the range 1.9-33.6 \% w/w, corresponding to osmotic pressures between $2.5 \mathrm{kPa}$ and $2 \mathrm{MPa}$.

All solutions and suspensions were prepared by mixing PEG or lyophilized microgels in milliQ water. Dialysis bags of molecular weight cut-off $6-8 \mathrm{kDa}$ were used (Spectra/Por 1, Spectrum). Initially, the bags are filled with a microgel suspension of $\sim 0.25 \mathrm{vol}$ fraction. As water escapes the bags during the process, it is necessary to refill the bags regularly with microgels (at least three times) in order to have enough material at the end of the compression. After each addition, the bag content is mixed manually. About one week after the last addition of microgels to the bag, the stressing PEG solution is renewed and the bags are allowed to equilibrate for one further week. The compressed microgel suspensions are then analyzed in terms of concentration and structure.

For comparison purposes, the osmotic pressure of solutions of non-crosslinked dexMA polymer and commercial dextran T40 ( 40 kDa Molecular weight, Pharmacosmos) was also measured as a function of concentration. The protocol was similar to the one previously described.

\section{Dextran concentration and microgel effective volume fraction}

After equilibration, the total dextran concentration in the bags was determined by drying a small amount of the sample at $105{ }^{\circ} \mathrm{C}$ until constant weight. At this temperature, the polymer does not decompose or vaporize and only water is removed from the sample. This way, we obtain a dry matter content $w_{p}$ which is the mass fraction of dextran polymer in the sample expressed in $\% \mathrm{w} / \mathrm{w}$. The measurements were repeated three times per sample and the variation between dry matter contents was below $1 \%$ for the same sample.

The dextran concentration in the samples can also be expressed in $g$ per volume of sample, which is simply obtained from dry matter content $w_{p}$ using the following relation: 


$$
C=\frac{w_{p}}{w_{p} / d_{\text {dex }}+\left(1-w_{p}\right) / d_{\text {water }}}
$$

This concentration $C$ is in turn used to define and calculate the effective volume fraction $\zeta$ :

$$
\zeta=n_{p} V_{p, 0}=\frac{C}{m_{p}} V_{p, 0}=\frac{C}{C_{i, 0}}
$$

with $n_{p}$ the number density of microgels, $V_{p, 0}$ and $m_{p}$ the native volume and dextran mass of one average microgel, respectively, and $C_{i, 0}$ the internal dextran concentration of a fully swollen, uncompressed, microgel (native internal dextran concentration in Table 1).

The use of an effective volume fraction $\zeta$ is now a standard in the microgel literature $[3,25,27] . \zeta$ is the volume fraction of microgels in the suspension as defined from their actual number density but with respect to their native, uncompressed size. The value of $\zeta$ is related to the degree of packing of the microgels in the suspensions. In the dilute regime, when the microgels are still not compressed and/or deformed, $\zeta$ is simply a measure of the true microgel volume fraction $(\phi)$ in suspension [17,27]. This holds until $\zeta$ reaches a limiting value at which the microgels start to deform and/or shrink. This limiting value can be related to the nature of the microgels [19,21] for instance for the specific case of ionic colloidal microgels) but is often close to the random close packing fraction for hard spheres, $\phi_{H S, R C P}$ We can then distinguish two theoretical regimes of compression after $\zeta$ reaches this limit: A first regime at $\phi_{H S, R C P} \lesssim \zeta<1$, where the microgels have the possibility to deform only and keep their initial volume, as compressed emulsion droplets would do for instance [50]. A second regime where $\zeta$ exceeds 1 and the microgels necessarily have to expel solvent to reach lower volumes than $V_{p, 0}$.

\section{Confocal laser scanning microscopy (CLSM)}

To image the microgels in the packings by CLSM, we create a contrast by adding FITC-dextran (500 kDa fluorescein-5-isothiocyanatedextran, Sigma-Aldrich) in the suspensions. FITC-dextran is too large to penetrate the microgel particles and the non-fluorescent microgels are visualized against a fluorescent background. To limit premature bleaching, the FITC-dextran is added at the last refill of the bags with microgels. The content of the bag is then mixed to assure an even distribution of the fluorescent polymer. Afterwards, the system is left to equilibrate for one more week to remove the water introduced with the FITC-dextran. We estimate the final concentration of FITC-dextran to be $\sim 0.2 \mathrm{mg}$ per $\mathrm{g}$ of sample. As we found 
experimentally (the osmotic pressures measured with dispersions that contains or not additional FITC-dextran were always virtually the same), the presence FITC-dextran at such a low concentration has no measurable impact on the osmotic resistance of the samples. It is then safe to consider that the images obtained using CLSM and with additional FITC-dextran are representative of the 'true' structure of the microgel packings without FITC-dextran.

To observe stacks of compressed microgels, we designed a sample holder with a depth equivalent to about ten times the diameter of the microgels. It consists of a glass slide in which a circular hole is drilled. The hole is $480 \mu \mathrm{m}$ deep and has $1 \mathrm{~cm}$ in diameter. The hole is manually filled with the sample and sealed with a glass coverslip and adhesive tape to avoid drying. Stacks of images with $1 \mu \mathrm{m}$ spacing are obtained using a Leica SP8 CLSM microscope (Plateforme Imagerie ToulouseRéseau-Imagerie).

For each sample, the images were treated using a home written MATLAB code that (i) calculates the volume fraction of voids in the packings, (ii) gives the distribution of microgel sizes by analyzing 200-800 particles. We provide additional information about the way the calculations are performed in Appendix A.

\subsection{Results}

\section{Osmotic pressure}

The osmotic pressures measured for the suspensions of Sephadex and dexMA microgels are given in Figure 1A and B, respectively (closed symbols). They are plotted as a function of dextran concentration $\mathrm{C}$ which is the total concentration of dextran polymer in the samples in $\mathrm{g}$ per volume. The pressures of solutions of dextran T40 (Figure 1A, open symbols) and methacrylated dextran T40 (Figure 1B, open symbols), as measured using the same methodology, are also given. In this way we compare the compression resistance of the microgel suspensions with the compression resistance of a reference polymer that corresponds to the noncrosslinked material of the microgels. Note that this is not strictly the case for Sephadex microgels as we do not know the exact size of the dextran precursor used in the making of these particles. However, Sephadex G25 and G100 are made from dextran with molecular weight $>>10 \mathrm{kDa}$ [61], and the osmotic pressure of dextran is relatively insensitive to dextran size at such molecular weights [62]. So, it is reasonable to consider dextran T40 as an adequate reference polymer for Sephadex here. 
G100-89 is the microgel with the highest initial water content in this study. It shows osmotic pressures that are always very close to the dextran T40 values, albeit systematically slightly lower (Figure 1A, green diamonds). We added in the figure vertical arrows that correspond to the positions of the reference points $\zeta=\phi_{H S, R C P}$ and $\zeta=1$. The first osmotic pressure point for G100-89 is located slightly below the RCP volume fraction for hard spheres, indicating that the suspension starts to resist compression before the microgels are closely packed. This is not an effect of thermal agitation as G100-89 consists of granular, non-Brownian, particles. Instead this is probably due to some frictional effects between the particles leading to the formation of stable loose packings at volume fractions below the RCP limit [63]. These packings resist compression as energy is needed to counteract frictional forces and go further up in microgel volume fraction. All the other pressure values for G100-89 correspond to $\zeta>1$ (second arrow), indicating that the microgels have necessarily deswollen and are probably strongly deformed and squeezed against each other, capable of reaching even an effective volume fraction $\zeta \approx 6$.

For microgel G25-68, the variation of osmotic pressure with polymer concentration is rather different (black squares in Figure 1A). At low concentration, the osmotic pressure is considerably lower than the pressures measured for dextran solutions. This difference is explained by the fact that suspensions of granular microgels start to resist compression only when the microgels come into contact and form a percolating network. Before that, the microgels sediment and there is no measurable resistance to this. As a result, we start to measure an osmotic pressure at a concentration $C$ that corresponds to $\zeta \approx \phi_{H S, R C P}$ (first arrow, about $200 \mathrm{~g} / \mathrm{L}$ ); the slight discrepancy towards lower concentrations is most probably because of interparticle frictional forces. The behavior of dextran solutions is rather different and show a measurable osmotic pressure as soon as polymer chains are present in solution, hence there is a large difference in pressure between dextran T40 and the microgels. As soon as we obtain a measurable osmotic pressure for the microgels, which is still at low concentrations, we observe a strong increase in osmotic pressure with $C$ (note the logarithmic scale). Interestingly, this increase occurs at concentrations that almost exactly matches the range $\phi_{H S, R C P}<\zeta<1$, meaning that the packing considerably gains in compression resistance when the microgels get into close contact and need to deform and/or deswell to accommodate the pressure. In a last regime, corresponding to $\zeta>1$, the increase in osmotic pressure with concentration becomes less pronounced and the pressures measured are close to those of dextran T40. At such high effective volume fractions, the microgels necessarily respond to compression by expelling water and shrinking to lower volume. 

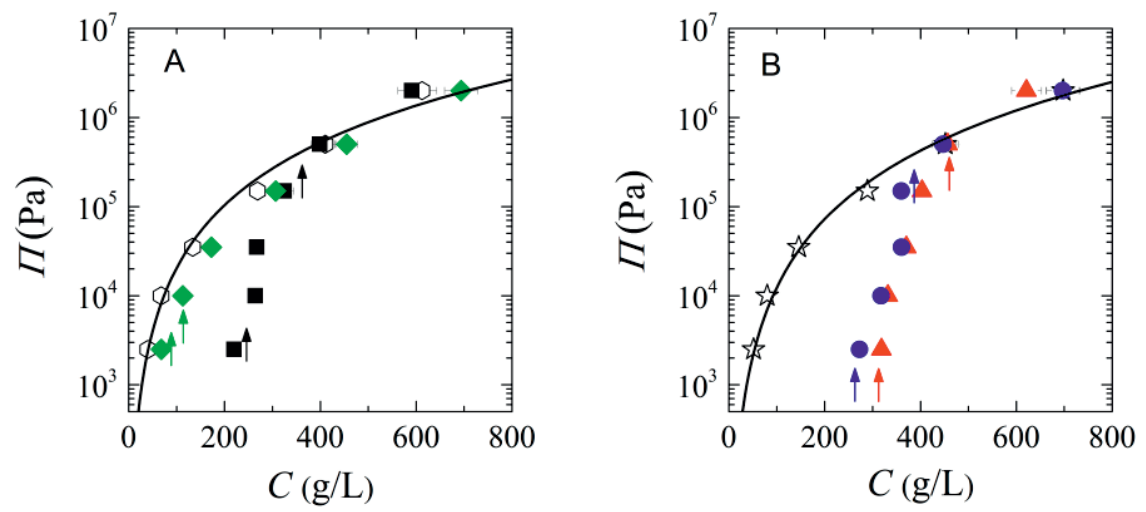

Figure 1. Osmotic pressure of dextran microgel packings and dextran solutions as a function of the overall polymer concentration in the system. A) dextran T40 (empty hexagons), Sephadex microgels G100-89 (green diamonds) and G25-68 (black squares). B) dexMA (empty stars), dexMA microgels MD-66 (blue circles) and MD61 (red triangles). The solid lines are power law fits to the osmotic pressure data of dextran T40 and dexMA. The arrows indicate the dextran concentration at which $\zeta=$ $\phi_{H S, R C P}$ (lower arrows) and $\zeta=1$ (higher arrows).

The results obtained with dexMA microgels are very similar to those obtained with Sephadex G25-68 particles, including the divergence in osmotic pressure when the particles get packed and the match between the pressure of the microgel suspensions and the pressure of the polymer material that constitutes the microgels at high concentrations (Figure 1B). We will not go into a detail description here but instead move to the next section in which we characterize the structure of the packings as a function of concentration.

\section{Images of the packings}

Figure 2 and Figure 3 present examples of CLSM images obtained for G100-89 and G25-68 microgels at the osmotic pressures reported in Figure 1. Images of compressed MD-61 and MD-66 microgel suspensions display features similar to those obtained for G25-68 and are given in Appendix A.

For all microgels, the pictures obtained at the lowest osmotic pressure correspond well to loose percolating packings of spherical or slightly deformed particles that oppose a small resistance to compression through interparticle frictional forces and/or particle deformation and deswelling. As osmotic pressure increases, the microgels get increasingly packed and deformed. This is more visible for G100-89 microgels that clearly lose their spherical shape in the range $0.60<\zeta<1.52$. 
Deformation is less obvious but still visible for the other microgels; mostly because the particles are of smaller size and/or the contrast is not optimal.

Note that the $500 \mathrm{kDa}$ FITC-dextran that we use as contrast agent has quite an unexpected behavior with G100-89 microgels (Figure 2). At low osmotic pressures $(\Pi \leq 35 \mathrm{kPa}$ ), it is effectively excluded from the particles because of its larger size compared to the average pore size of the microgels $[55,64]$. However, at large osmotic pressures $(\Pi \geq 150 \mathrm{kPa})$, when the microgels are closely packed and deformed against each other and the FITC-dextran clearly enters the structure of the microgels. This phenomenon is particularly difficult to interpret. At first sight, it is tempting to explain this through the effective degree of entanglement experienced by the FITC-dextran when the interstitial voids between the microgels are closed. At some point, it is indeed plausible that the interstitial concentration in FITC-dextran reaches a limit where entanglement effects are so strong that it becomes more favorable for the FITC-dextran to enter the microgels through reptation (a similar effect is described in $[65,66]$ for instance). This mechanism can perfectly be translated in terms of osmotic pressure: when the osmotic pressure of FITC-dextran becomes higher than the osmotic pressure of the microgels - which is equal to the osmotic pressure of the PEG reservoir - some of the FITC-dextran enters the microgel to equilibrate the pressures inside and outside the microgels. However, we find that this whole explanation based on the effective concentration in FITC-dextran in the voids is not satisfactory for at least two reasons:

(i) the total concentration of FITC-dextran in the samples is very low, $\sim 0.2$ $\mathrm{mg} / \mathrm{g}$, which leads to a maximum concentration of $\sim 15-50 \mathrm{~g} / \mathrm{L}$ in the interstitial voids using the void fraction values of Figure 4B (see next paragraph). This concentration of FITC-dextran in turn corresponds to an osmotic pressure of about $2 \mathrm{kPa}$ (dextran tabulated values of Peter Rand [67]), which is much lower than the pressures at which the FITCdextran enters the microgels.

(ii) the fluorescence intensities suggest that FITC-dextran has entered the microgels in such a way that it is more concentrated inside the microgels than in the interstitial voids. Such a 'reverse' exclusion phenomenon clearly cannot be explained through osmotic/entanglement effects only, but is more probably the results of some kind of attractive interactions between the tracer and the microgel. 

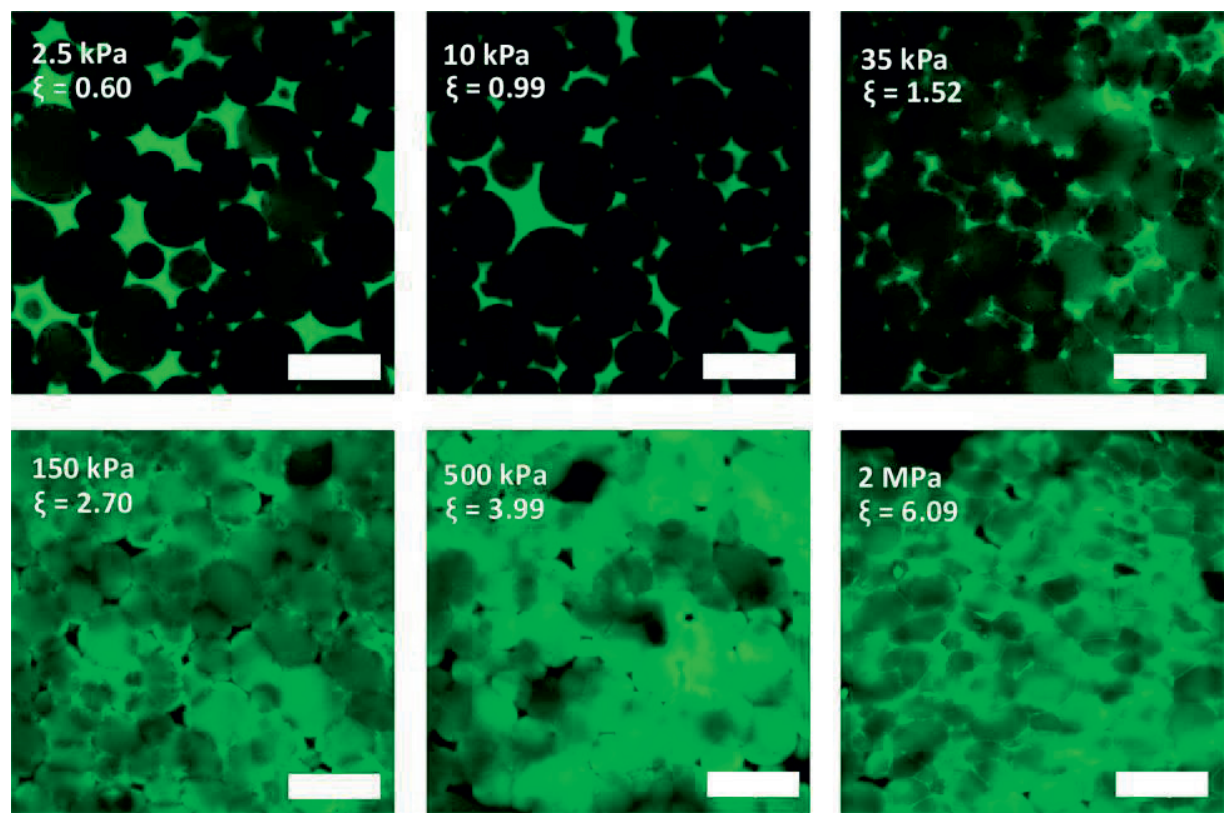

Figure 2. Confocal Laser Scanning Microscope images of compressed G100-89 microgels. The scale bar is $100 \mu \mathrm{m}$.
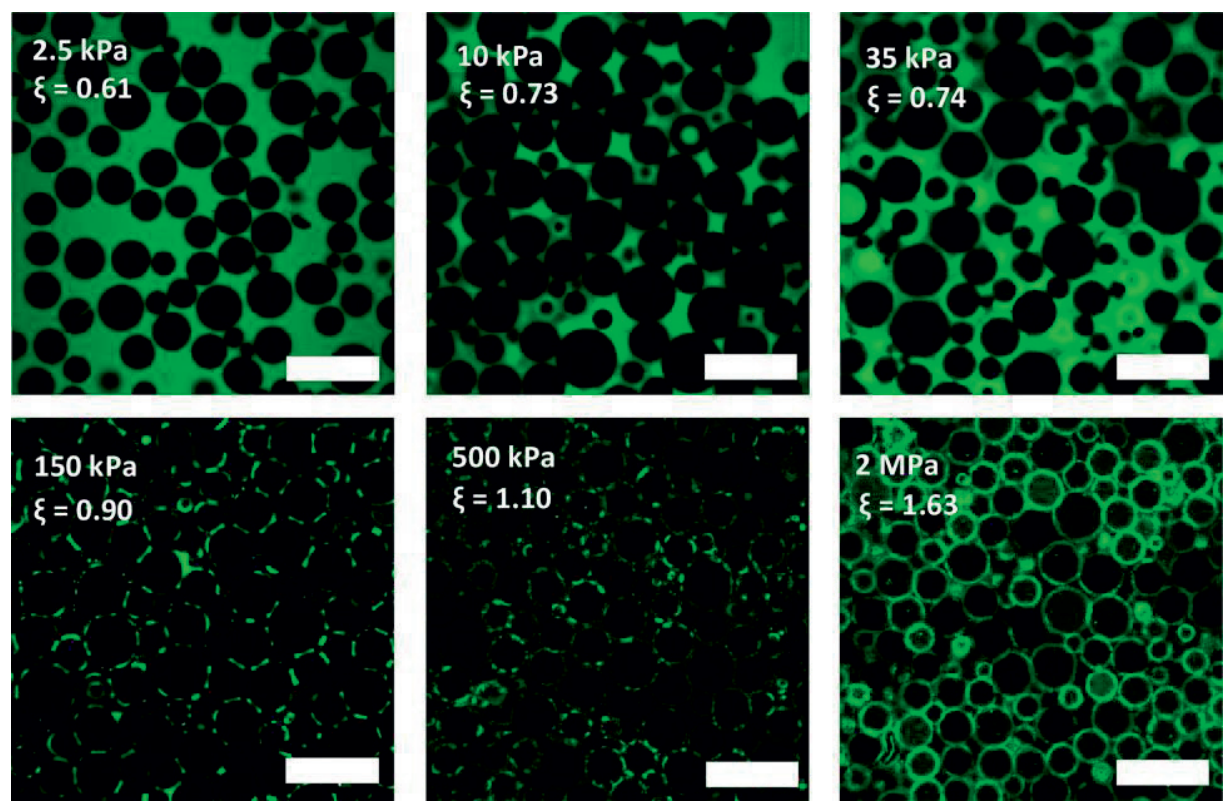

Figure 3. CLSM images of compressed G25-68 microgels. The scale bar is $100 \mu \mathrm{m}$. 
We will not go further into this discussion here, as it is clearly beyond the scope of this study to properly understand the reasons for this accumulation of FITC-dextran in the microgels in conditions of dense packing. We stress however that we do not observe this effect with the Sephadex G25-68 microgels, except maybe at the highest osmotic pressure where the FITC-dextran seems to have started to diffuse in the periphery of the particles (Figure 3,2 MPa). For dexMA particles, we observe penetration of FITC-dextran at compression pressures $\geq 500 \mathrm{kPa}$ only (Appendix A).
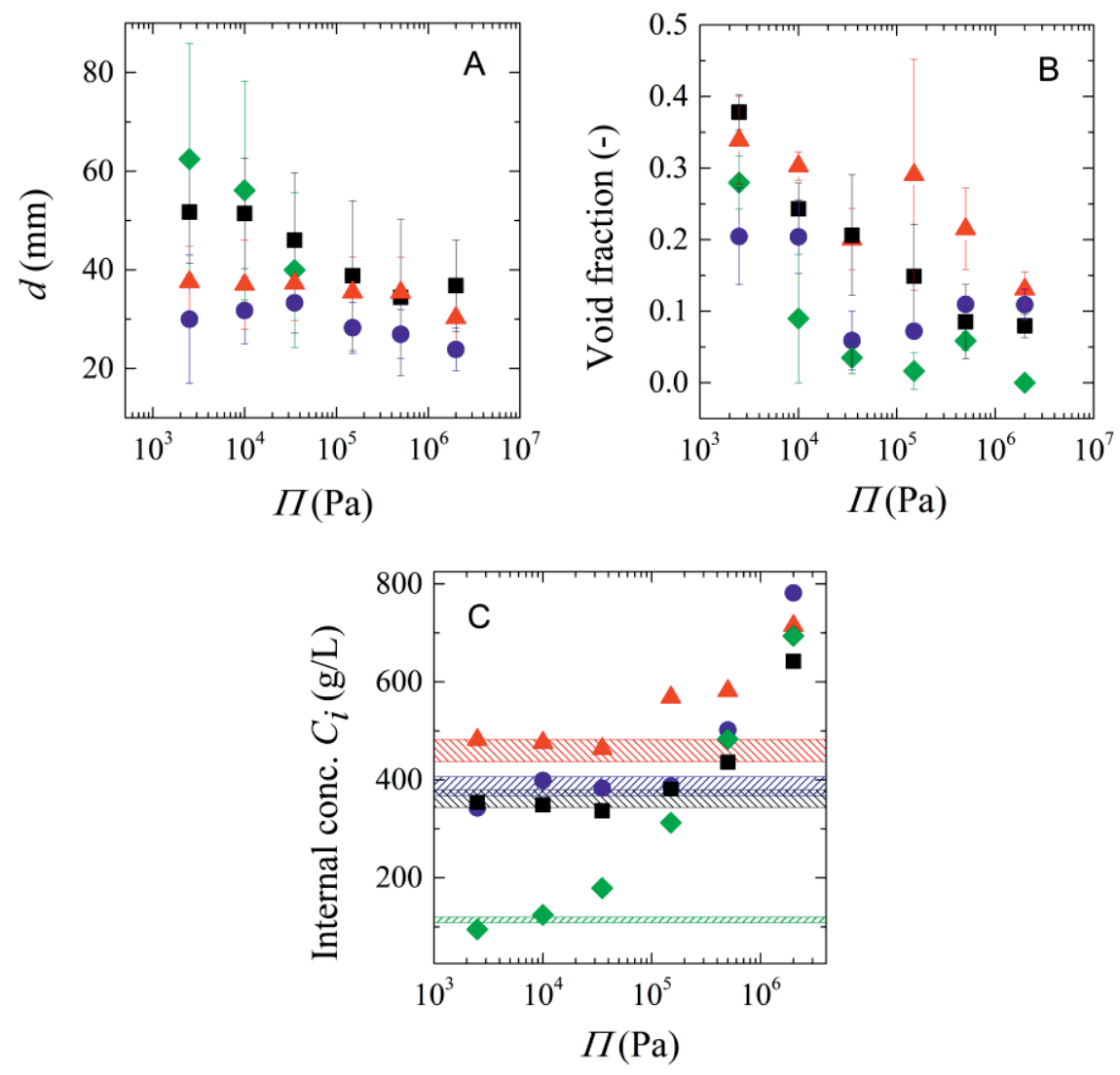

Figure 4. (A) Microgel mean diameter as a function of osmotic pressure. (B) Fraction of void in the packings as a function of osmotic pressure. (C) The evolution of the internal dextran concentration of the microgels in the packings as calculated from the void fraction data (Eq. (4). The patterned areas correspond to the native internal concentration of the microgels at zero pressure $\left(C_{i, 0}\right.$ in Table 1$)+/-5 \%$. Microgels G100-89 (green diamonds), G25-68 (black squares), MD-66 (blue circles) and MD-61 (red triangles). 
An interesting question is how much the individual microgel particles have actually squeezed in the packings: do they always squeeze and loose internal water when the packings are compressed or is there a regime at which they only deform at constant volume like emulsion droplets do for instance? To answer this question, one can first look at the evolution of the size of the particles as measured from the CLSM images (Figure 4A). The reported sizes are the average diameters obtained by analyzing 200 800 particles in each sample. This analysis also suggests that there is no significant evolution of size polydispersity with compression for each microgel population. However, we chose not to present these results as it would require more statistics and/or more advanced characterization techniques (like SAXS or SANS in the case of colloidal particles for instance $[21,22]$ ) to investigate this question properly.

The decrease in size is obvious for the G100-89 microgels and starts already at the lowest osmotic pressures (the values at high $\Pi$ values are not reported as the microgels are then highly deformed and the size of individual particles cannot be determined precisely). This is in line with effective volume fractions $\zeta \geq 1$ being reached early in the compression process (second point of osmotic pressure) and that the particles necessarily expel water. For the other three other microgels, the size is not really affected by compression at low pressures and the decrease in size is only apparent at pressures $\geq 150 \mathrm{kPa}$. This suggests that the microgel particles loose volume and expel water only in this second range of applied pressures.

To confirm that, we now look at the volume fraction occupied by the interstitial voids that persist in the packing during compression. This void fraction can be measured from the CLSM images with quite good precision and is plotted as a function of osmotic pressure in Figure 4B. As expected, we see that the voids get progressively closed with compression without disappearing completely, except for G100-89 for which the voids vanish. What is interesting here is that we can estimate the actual degree of squeezing of the microgels from the measured void fractions. Indeed, the internal polymer concentration of the microgels in the packings is simply given by:

$$
C_{i}=\frac{C}{(1-\text { void fraction })}
$$

In Figure $4 \mathrm{C}, C_{i}$ is plotted as a function of osmotic pressure. For G100- 89, $C_{i}$ exceeds the native internal dextran concentration $C_{i, 0}$ of the microgels at pressures $\geq 10 \mathrm{kPa}$. This confirms that the particles start to expel water from this pressure upward, in accordance with the changes in size of Figure 4A. For the other microgels, $C_{i}$ in found to be very close to $C_{i, 0}$ for the first 3-4 points of osmotic pressure, meaning that the 
particles have not yet expelled water in that range. $C_{i}$ goes beyond $C_{i, 0}$ only at high osmotic pressures, as expected from the changes in size shown in Figure 4A.
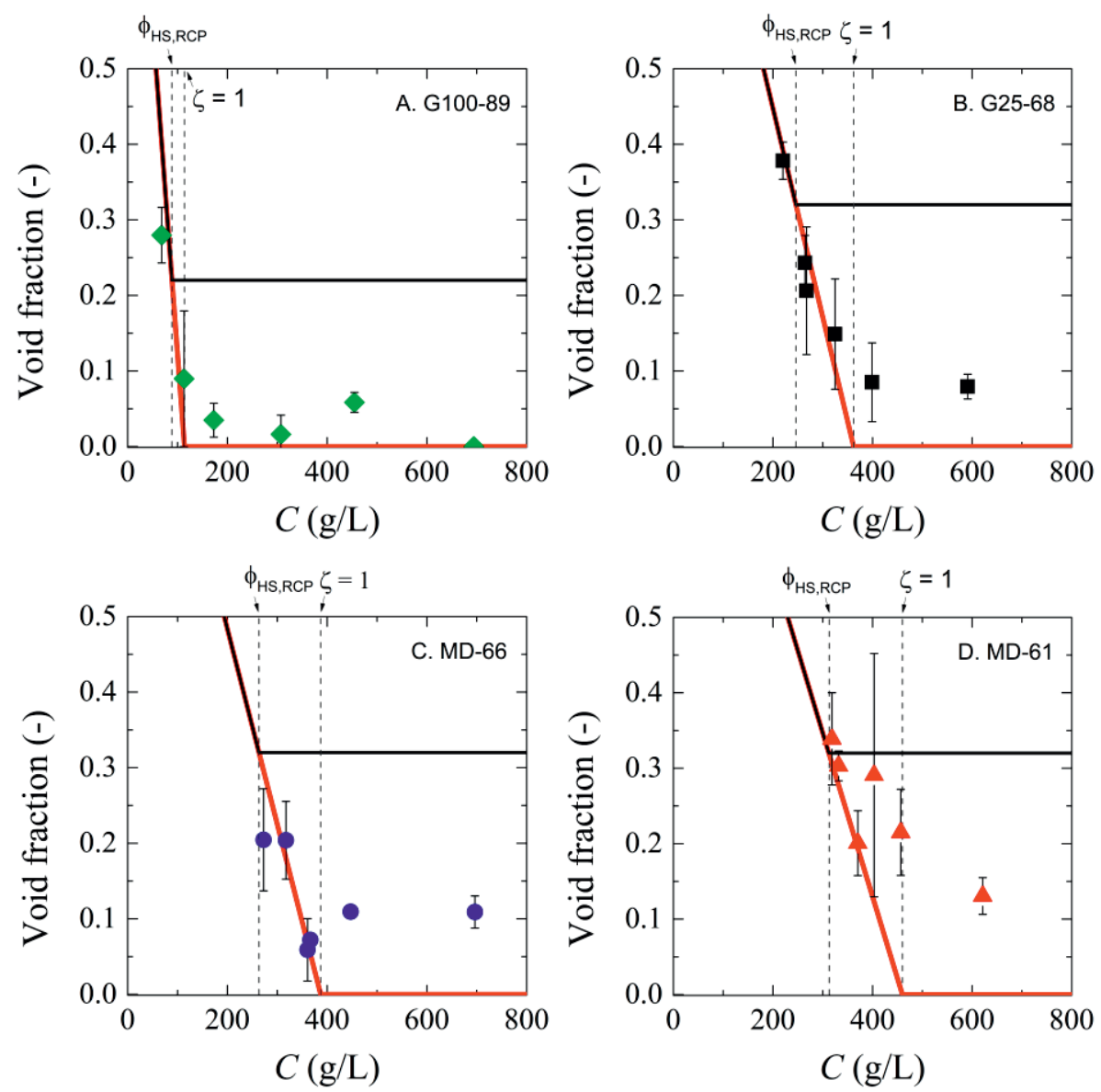

Figure 5. Evolution of the void fraction in the packings as a function of the average dextran concentration in the system. The black line is the theoretical evolution of void fraction for packings of particles that do not deform but only deswell at $\zeta \geq \phi_{H S, R C P}$ (void fraction $=1-C / C_{i, 0}$ at $\zeta<\phi_{H S, R C P}$ and void fraction=1- $\phi_{H S, R C P}$ at $\zeta \geq$ $\left.\phi_{H S, R C P}\right)$. The red line is the theoretical evolution of the void fraction for packings of particles that only deform and do not deswell, such as emulsion droplets (void fraction $=1-C / C_{i, 0}$ until vanishing). 
Another interesting way of looking at these results is by comparison of the observed void fraction as a function of $C$, i.e. the average dextran concentration in the suspension (Figure 5), with 'ideal' scenarios. Particles that cannot deform at all and only deswell are represented by the black lines, while objects that can only deform without losing volume before $\xi=1$ (such as emulsions) are represented by red lines.

We clearly see in Figure 5 that all dextran microgels have the tendency to follow the behavior of deformable but non-compressible objects in the range $\phi_{H S, R C P} \leq \zeta \leq 1$. At $\zeta \geq 1$, the compressibility of the microgels then comes into play and the particles squeeze to smaller volumes, while some voids persist. The presence of these remaining voids is an important point that we discuss further in the following section.

\subsection{Summary and discussion}

The results presented above can be summarized as follows:

(1) Suspensions of dextran, granular microgels start to resist compression at a volume fraction that matches the random close packing of hard-spheres of similar size distribution, $\phi_{H S, R C P}$. This is consistent with the fact that these microgels are non-Brownian particles that sediment and come into contact at $\phi \approx \phi_{H S, R C P}$ with no measurable resistance. In two cases (Sephadex), the volume fraction at which the packings start to resist compression is slightly below $\phi_{H S, R C P}$, which may be caused by some frictional forces between the microgels [63]. Note that this general behavior of granular and neutral microgels at low volume fractions is very different from the one of colloidal scale microgels where particle-particle interactions of different types (hardsphere like, electrostatic), and in some cases counterions, produce a measurable osmotic resistance before closepacking [19,21,22,46-49].

(2) In a first regime of compression, at effective volume fractions between $\phi_{H S, R C P}$. and 1 , the compression resistance rises from zero to a value that is close to the resistance of a dextran solution of the same average concentration. Images of the packings in that range of concentration indicate that the microgels increasingly pack and deform with compression, while the internal dextran concentration is close to the native one. So, the microgels behave like objects that only deform and do not loose internal volume upon compression, as in the case for emulsions for instance. A similar behavior is reported in Chapter 3 with polyacrylamide microgel particles of slightly smaller size $(\sim 10 \mu \mathrm{m})$. Interestingly, the fact that shape deformation dominates over squeezing in a first regime of compression was also recently observed for colloidal pNIPAM microgels [26]. In that case 
however, interpenetration effects are also significant [25,26]; while such effects are not visible in the present work. We note finally that compression never leads in our case to highly ordered crystal-like structures, as it was observed with colloidal and polydisperse polyelectrolyte microgels $[21,22,29,30]$.This is probably because the size distribution of the microgels is relatively unchanged upon compression in our case, while for pNIPAM colloidal microgels, charge effects makes the largest microgels deswell before the smaller ones, thus decreasing size polydispersity and inducing crystallization [22].

(3) In a second regime of compression, at effective volume fractions $\zeta \geq 1$, the resistance of the packings to compression becomes similar to that of a homogeneous solution of the polymer that constitutes the microgels. In this regime, the only way to concentrate the system is to compress the individual microgels in the packing. The microgels are strongly deformed and squeezed, leading to reduction of size, and increased internal dextran concentration. The deformation is however not sufficient to close all interstitial spaces in the packings. This is here an interesting and potentially important difference with colloidal scale microgels where the persistence of interstitial voids in highly dense packings is usually not considered or observed $[3,22,26]$.

Next, we focus on points (2) and (3) and look for qualitative and/or quantitative explanations for our results using existing theoretical frameworks.

At concentrations between $\zeta=\phi_{H S, R C P}$ and $\zeta=1$, the microgels mainly deform upon compression, and seem to act similarly to emulsions, which we take as a reference case. The osmotic resistance of concentrated emulsions was investigated by Mason and co-workers in the late 1990s $[50,68]$. These authors propose a 'network spring model' to describe the osmotic pressure at $\zeta>\phi R C P$ [68]. In this model, the pressure depends on the number of facets formed between neighboring droplets in the packing. Any additional facet behaves as a spring that further resists compression. The number of springs between neighboring droplets grows as $\sim\left(\zeta-\phi_{R C P}\right)$, while the number of droplets per unit volume grows with $\zeta$. We then have:

$$
\Pi \sim \zeta\left(\zeta-\phi_{H S, R C P}\right)
$$

For all the microgels investigated, we find that the experimental osmotic pressure qualitatively follows the emulsion model in the concentration range $\phi_{H S, R C P}<\zeta<1$ (Figure 6A-D). This suggests that the resistance to compression of the microgel 
packings has a similar origin as that of emulsions: a network of facets acting like springs between neighboring microgels. For emulsions, the spring constant is given by the droplets surface tension [68], while for microgels packings, it is the elastic modulus of the microgel particles that sets the force of theses springs [69].

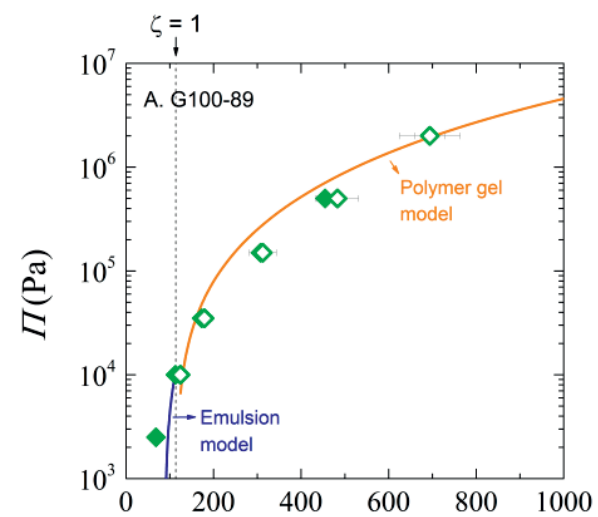

Polymer concentration $C, C_{i}(\mathrm{~g} / \mathrm{L})$

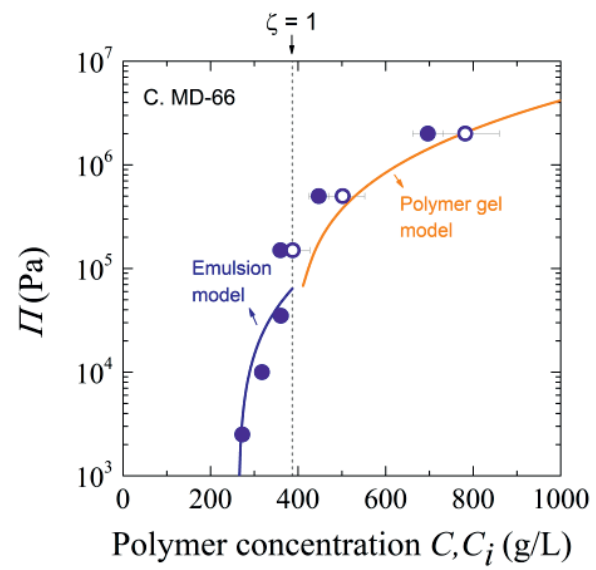

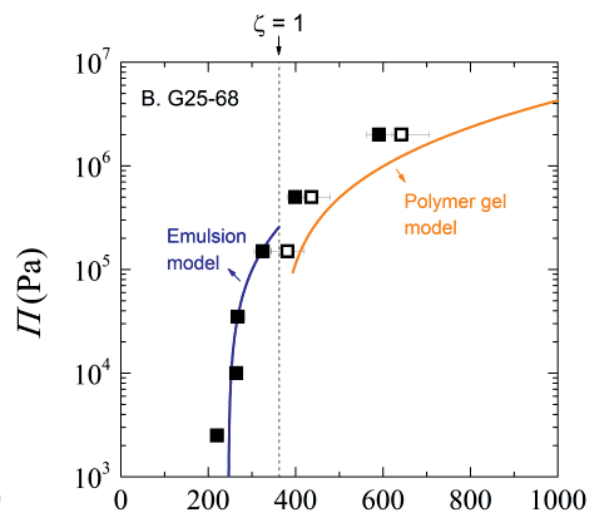

Polymer concentration $C, C_{i}(\mathrm{~g} / \mathrm{L})$

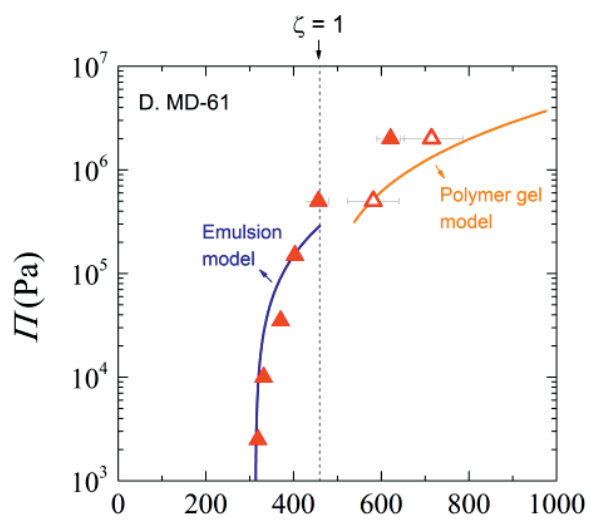

Polymer concentration $C, C_{i}(\mathrm{~g} / \mathrm{L})$

Figure 6. Compression resistance of the microgel packings as a function of the average polymer concentration in the system $C$ (closed symbols) or the internal concentration of the microgels $C_{i}$ (empty symbols). At low effective volume fractions $\zeta \leq 1$, the packings mostly resist through deformation of the microgels and $\Pi$ increases with $C$ as it does for concentrated emulsions (blue line, [50]). At high effective volume fraction $\zeta \geq 1$, the systems mostly respond to compression by expelling water from the particles. This time $\Pi$ increases with $C_{i}$ as it does for a reticulated polymer gel of properties identical to the microgel material (orange line, [45]). 
At $\zeta>1$, the microgels reduce their size and expel water, as reported in Figure 4A and $\mathrm{C}$. On the other hand, the void fraction in the packing does not totally vanish (Figure 4B and Figure 5), suggesting that deformation is not preponderant in this regime. So, as a first approximation, we consider the resistance to compression of the packings to be essentially due to the resistance of the individual microgels to deswelling. In that case, the Flory-Rehner (FR) theory for the osmotic pressure of connected gels is directly applicable [2,40-45]. The theory describes the osmotic pressure $\Pi$ as a sum of a mixing contribution $\Pi_{m}$ and an elastic contribution $\Pi_{e l}$. The mixing contribution results from the entropy of mixing of the polymer segments with the solvent. It corresponds to the osmotic pressure of the dextran polymers that we measure experimentally (empty symbols in Figure 2). These osmotic pressures are described by:

$$
\Pi_{m}=a C^{b}
$$

with $\Pi$ in $\mathrm{Pa}, C$ in $\mathrm{g} / \mathrm{L}$, and $a=0.420$ or 0.095 and $b=2.34$ or 2.56 for dextran $\mathrm{T} 40$ and dexMA, respectively. Such simple empirical power law expressions are commonly used for describing the osmotic pressure of polymers [70].

The elastic contribution results from the presence of crosslinks that prevent the polymer gel from fully swelling and dissolving. It is a negative contribution to the osmotic pressure that can be approximated using the following equation [45]:

$$
\Pi_{\text {el }}=-\frac{d_{\text {dex }} N_{A} k_{B} T}{N_{x} M_{\text {monomer }}} \phi_{\text {ref }}\left[\frac{2}{f} \frac{\phi}{\phi_{\text {ref }}}-\left(\frac{\phi}{\phi_{\text {ref }}}\right)^{1 / 3}\right]
$$

with $N_{A}$ the Avogadro number, $k_{B}$ the Boltzmann constant, $T$ the temperature, $N_{x}$ the number of monomers between crosslinks, $M_{\text {monomer }}$ the molecular mass of the monomer $(180 \mathrm{~g} / \mathrm{mol})$, and $f$ the functionality of the crosslinks (taken as $f=4)$. $\phi$ is the polymer volume fraction in the gel matrix, while $\phi_{r e f}$ is the polymer volume fraction at a reference state, generally taken as when the chains between the crosslinks are fully relaxed (for a critical review about the definition of $\phi_{r e f}$, we refer the reader to [44,71]). For gels of crosslinked dextran and similar polymers, Van der Sman finds using FR theory that $\phi_{r e f} \approx 2 / 3 \phi_{0}$, with $\phi_{0}$ the polymer volume fraction of the fully swollen gel at zero osmotic pressure [45]. $\phi_{0}$ can be directly calculated from $C_{i, 0}$ (Table 1), using $\phi_{0}=C_{i, 0} / d_{d e x} . N_{x}$ is calculated for each microgel using the native dextran concentration in the fully swollen state, where $\Pi=0$ and therefore $\Pi_{m}\left(C_{i, 0}\right)=$ $-\Pi_{e l}\left(\phi_{0}\right)$. 
This model is now compared with the osmotic pressure data plotted as a function of the internal concentration of the microgels (open symbols in Figure 6), as it is this concentration that determines the resistance to compression in the framework of the Flory-Rehner theory. The agreement between the model and our experimental data is quite satisfactory in all cases. This suggests that we have found a way to predict the osmotic resistance of the packings based on the knowledge of the internal concentration of the microgels. This is a subtle but important difference with highly compressed packings of microgels that no longer contain voids, like those studied by Menut et al. for instance [3]. For these packings, the average polymer concentration obviously matches the internal concentration of the microgels, and the osmotic pressure can directly be predicted with Flory-Rehner theory using the average concentration in the system $[3,45]$. When dealing with microgel packings with voids, as in the present work, the prediction is complicated by the necessity to determine the actual degree of squeezing from which the internal polymer concentration needs to be derived.

\subsection{Conclusions}

In this chapter, we report on the behavior of suspensions of granular scale dextran microgels when exposed to an external osmotic pressure. Our experiments assess the resistance of the packings to compression, as well give qualitative and quantitative information about the structure of the packings. As expected for granular, nonBrownian microgels, the resistance to compression starts to rise at the vicinity of the volume fraction of random close-packing. In a first range of compressions that exceed this value, the microgels mainly deform, leading to a strong rise in resistance, in analogy with emulsion systems. In a second higher regime of compression, the microgels mainly respond to compression by expelling water. The resistance to compression of these systems can be estimated through a Flory-Rehner model based on the actual polymer concentration inside the microgel particles, therewith taking into account the presence of persistent voids in the packings.

With these results, we demonstrate that loose to dense packings of neutral and granular-scale microgels clearly do not respond to compression as a uniform gel of the same material at the same average concentration; an analogy that has been used so far in literature for very dense packings of colloidal microgels where persistent voids are inexistent [3]. As a perspective, it would be interesting to focus on the resistance to compression of more complex systems like packings of colloidal-sized polyelectrolyte microgels for instance, in relation with effects that were recently reported with such systems, e.g. interpenetration [25] and crystallization [22]. This would lead to a better understanding of how a collection of deformable and 
compressible particles resist to an increase in concentration, depending on the size and architecture of the involved particles. Such information would in turn be highly useful for predicting concentration operations, e.g. filtration of microgels, in which resistance determines overall productivity.

\section{Acknowledgements}

We thank Cécile Pouzet and Alain Jauneau for technical assistance in the use of the CLSM instrument, as well as Emmanuel Cid (LGC) for his scientific and technical support for the treatment of the CLSM images. 


\section{Appendix A. Supplementary data Synthesis of dextran (dexMA) microgels}

Methacrylated dextran (dexMA) microgels are prepared at two different water contents. DexMA, the microgel precursor, is first synthesized by a transesterification reaction of dextran T40 (Pharmacosmos) and glycidyl methacrylate (Sigma-Aldrich) following the method of van Dijk-Wolthuis [72,73]. Then dexMA and polyethylene glycol (PEG) 10kDa (Sigma-Aldrich) solutions are mixed with a three-bladed propeller stirrer at $1500 \mathrm{rpm}$ at a temperature of $25^{\circ} \mathrm{C}$. As the two solutions are partially immiscible, a phase separation occurs upon mixing, giving a dispersed droplet phase, rich in dexMA and poor in PEG, and a continuous phase rich in PEG and poor in dexMA [52,53]. N,N,N',N'-tetramethylenediamine (TEMED) (SigmaAldrich; 20\% w/w, pH neutralized with HCL 4M) is added as an initiator for the crosslinking reaction. The stirring speed is decreased to $500 \mathrm{rpm}$ and potassium peroxodisulfate (KPS) (Sigma) is added to crosslink the dextran chains and to turn the dexMA droplets into microgel particles. The suspension of microgels is centrifuged and washed five times with water to remove the supernatant rich in PEG and obtain a suspension of microgels in water ( $3000 \mathrm{~g}$ for 5 minutes for the first wash and $500 \mathrm{~g}$ for 1 minute for the four next washes). The microgels are freeze dried before storage. 


\section{Treatment of CLSM images}

Void fraction. The pictures are first converted to binary. The number of black (particles) and white (void) pixels in each image are then counted. As the number of images in a stack and the distance between images is known, it is possible to calculate the total volume of the stack and the total volume occupied by the white and black pixels. The void fraction is simply:

$$
\text { Void fraction }=\frac{\text { number of white voxels }}{\text { total number of voxels }}
$$

Microgel size. The microgels of a given stack are identified. For each of them, a radius $r_{1}$ and a radius $r_{2}$ is measured on two 2D-images of the stack. The true radius of the particle is then obtained as shown in Figure 7 and using the two following equations [74]:

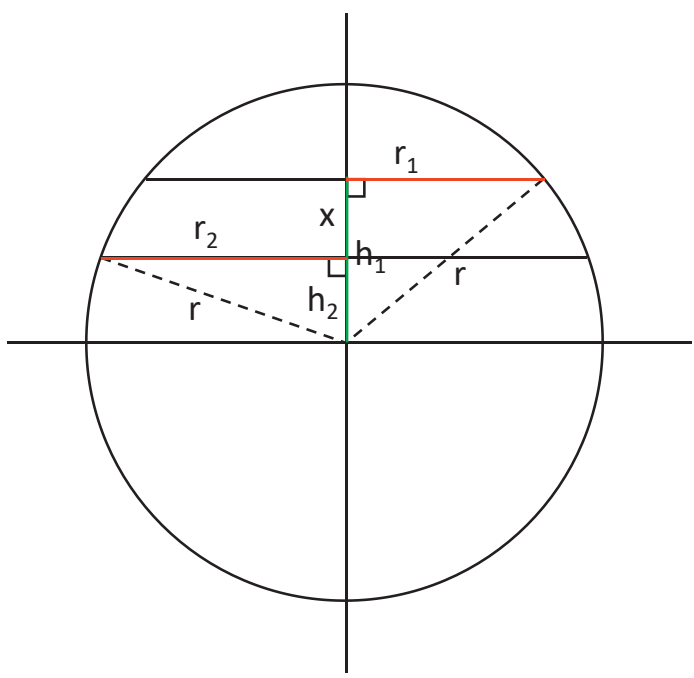

Figure 7. Particle radius calculation from two slices in a stack.

$$
\begin{gathered}
h_{1}=h_{2}+x \\
r=\sqrt{{h_{1}{ }^{2}+r_{1}{ }^{2}}^{2}}=\sqrt{{h_{2}{ }^{2}+r_{2}{ }^{2}}^{2}}
\end{gathered}
$$

where $x$ is the known distance between the images in the stack. 
CLSM images of compressed dexMa microgel suspensions
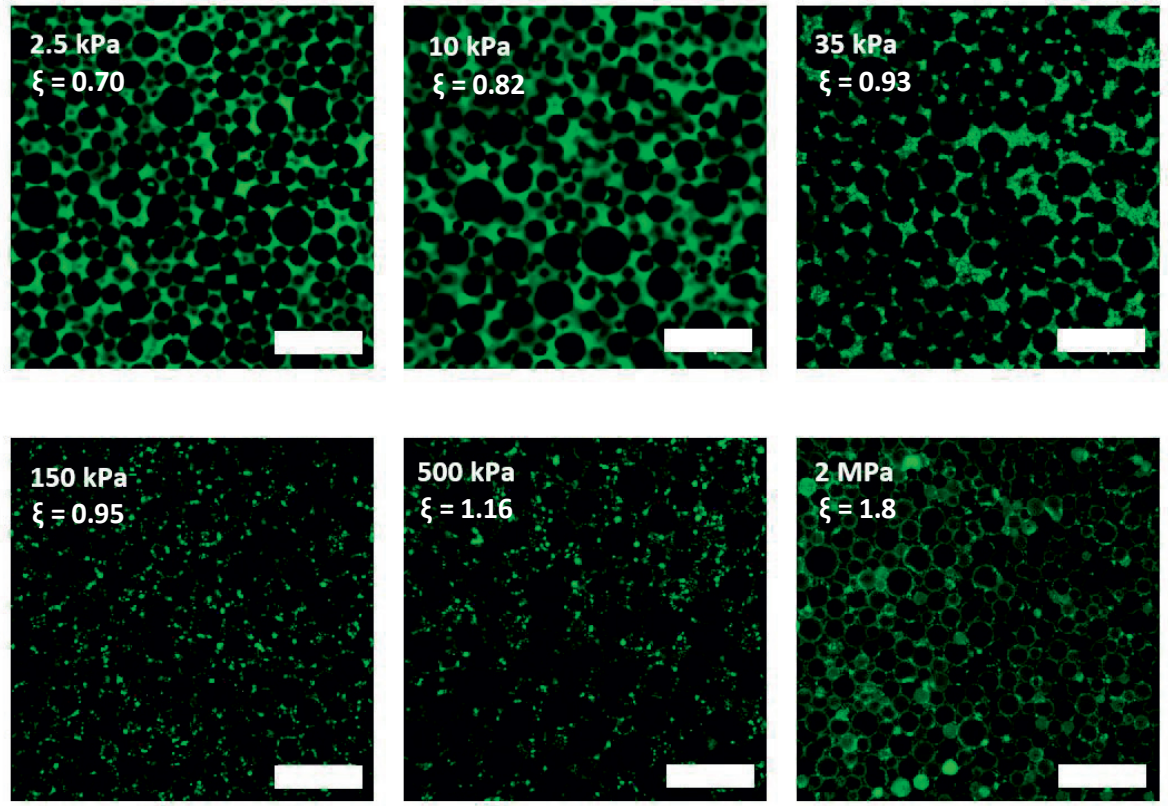

Figure 8. CLSM images of compressed MD-66 microgels. Scale bar is $100 \mu \mathrm{m}$.
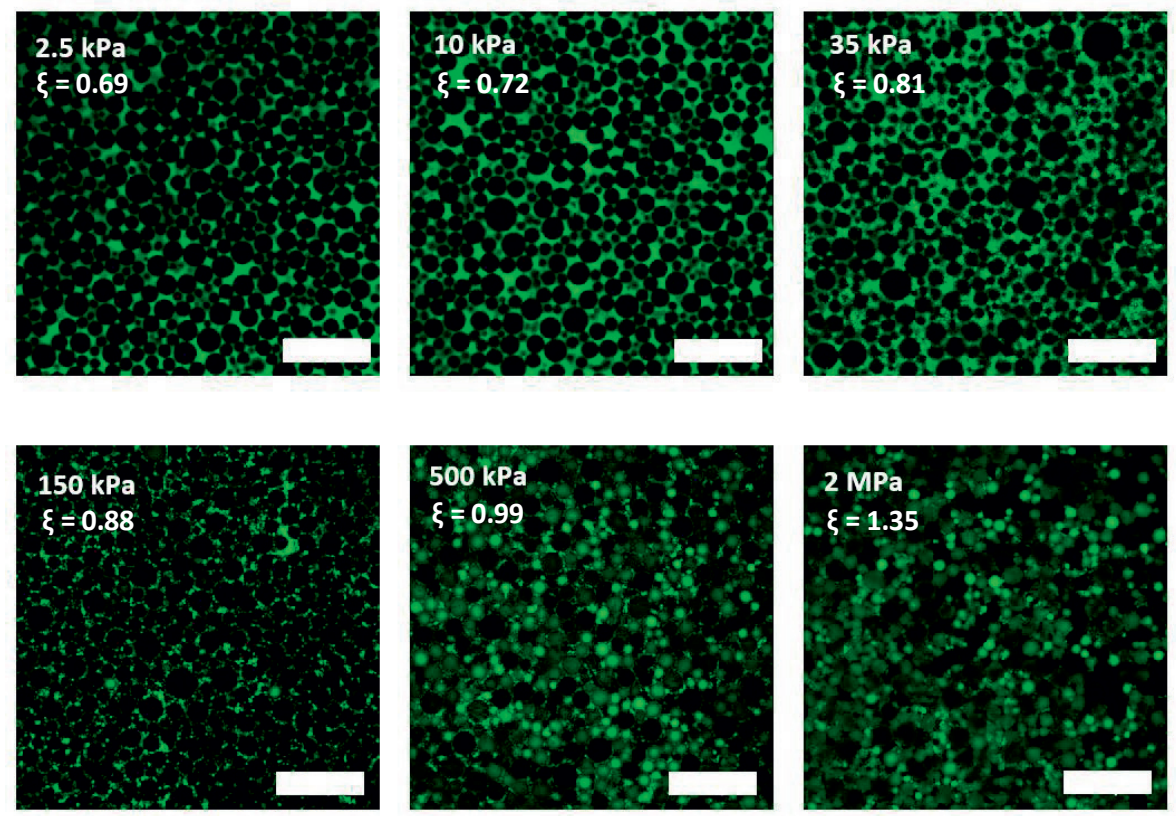

Figure 9. CLSM images of compressed MD-61 microgels. Scale bar is $100 \mu \mathrm{m}$. 


\section{References}

[1] F.A. Plamper, W. Richtering, Functional microgels and microgel systems, Acc. Chem. Res. 50 (2017) 131-140.

[2] J.J.F. Sleeboom, P. Voudouris, M.T.J.J.M. Punter, F.J. Aangenendt, D. Florea, P. Van der Schoot, H.M. Wyss, Compression and reswelling of microgel particles after an osmotic shock, Phys. Rev. Lett. 119 (2017) 098001.

[3] P. Menut, S. Seiffert, J. Sprakel, D.A. Weitz, Does size matter? Elasticity of compressed suspensions of colloidal- and granular-scale microgels, Soft Matter. 8 (2012) 156-164.

[4] B.R. Saunders, B. Vincent, Microgel particles as model colloids: theory, properties and applications, Adv. Colloid Interface Sci. 80 (1999) 1-25.

[5] B.R. Saunders, N. Laajam, E. Daly, S. Teow, X. Hu, R. Stepto, Microgels: from responsive polymer colloids to biomaterials, Adv. Colloid Interface Sci. 147148 (2009) 251-262.

[6] J.K. Oh, R. Drumright, D.J. Siegwart, K. Matyjaszewski, The development of microgels/nanogels for drug delivery applications, Prog. Polym. Sci. 33 (2008) 448-477.

[7] E. Dickinson, Microgels - an alternative colloidal ingredient for stabilization of food emulsions, Trends Food Sci. Technol. 43 (2015) 178-188.

[8] A. Bouchoux, P. Qu, P. Bacchin, G. Gésan-Guiziou, A general approach for predicting the filtration of soft and permeable colloids: the milk example, Langmuir 30 (2014) 22-34.

[9] O. Nir, T. Trieu, S. Bannwarth, M. Wessling, Microfiltration of deformable microgels, Soft Matter. 12 (2016) 6512-6517.

[10] W.M. Lu, K.L. Tung, C.H. Pan, K.J. Hwang, Crossflow microfiltration of mono-dispersed deformable particle suspension, J. Membr. Sci. 198 (2002) 225243.

[11] K.J. Hwang, C.L. Hsueh, Dynamic analysis of cake properties in microfiltration of soft colloids, J. Membr. Sci. 214 (2003) 259-273.

[12] K. Horigome, D. Suzuki, Drying mechanism of poly(N-isopropylacrylamide) microgel dispersions, Langmuir 28 (2012) 12962-12970.

[13] C. Sadek, L. Pauchard, P. Schuck, Y. Fallourd, N. Pradeau, C. Le Floch-Fouéré, R. Jeantet, Mechanical properties of milk protein skin layers after drying: understanding the mechanisms of particle formation from whey protein isolate and native phosphocaseinate, Food Hydrocoll. 48 (2015) 8-16.

[14] M. Cloitre, R. Borrega, F. Monti, L. Leibler, Structure and flow of polyelectrolyte microgels: from suspensions to glasses, Comptes Rendus Phys. 4 (2003) 221-230. 
[15] C. Pellet, M. Cloitre, The glass and jamming transitions of soft polyelectrolyte microgel suspensions, Soft Matter. 12 (2016) 3710-3720.

[16] C.L.A. Berli, D. Quemada, Rheological modeling of microgel suspensions involving solid/liquid transition, Langmuir 16 (2000) 7968-7974.

[17] A. Bouchoux, B. Debbou, G. Gesan-Guiziou, M.H. Famelart, J.L. Doublier, B. Cabane, Rheology and phase behavior of dense casein micelle dispersions, J. Chem. Phys. 131 (2009) 165106-165111.

[18] P. van der Scheer, T. van de Laar, J. van der Gucht, D. Vlassopoulos, J. Sprakel, Fragility and strength in nanoparticle glasses, ACS Nano 11 (2017) 6755-6763.

[19] M. Pelaez-Fernandez, A. Souslov, L.A. Lyon, P.M. Goldbart, A. FernandezNieves, Impact of single-particle compressibility on the fluid-solid phase transition for ionic microgel suspensions, Phys. Rev. Lett. 114 (2015) 098303.

[20] U. Gasser, J.-J. Lietor-Santos, A. Scotti, O. Bunk, A. Menzel, A. FernandezNieves, Transient formation of bcc crystals in suspensions of poly $(\mathrm{N}$ isopropylacrylamide)-based microgels, Phys. Rev. E 88 (2013) 052308.

[21] A. Scotti, U. Gasser, E.S. Herman, M. Pelaez-Fernandez, J. Han, A. Menzel, L.A. Lyon, A. Fernández-Nieves, The role of ions in the self-healing behavior of soft particle suspensions, Proc. Natl. Acad. Sci. 20 (2016) 5576-5581.

[22] A. Scotti, U. Gasser, E.S. Herman, J. Han, A. Menzel, L.A. Lyon, A. FernandezNieves, Phase behavior of binary and polydisperse suspensions of compressible microgels controlled by selective particle deswelling, Phys. Rev. E 96 (2017) 032609.

[23] G. Romeo, L. Imperiali, J.W. Kim, A. Fernandez-Nieves, D.A. Weitz, Origin of deswelling and dynamics of dense ionic microgel suspensions, J. Chem. Phys. 136 (2012) 124905-124909.

[24] I. Bouhid de Aguiar, T. Laar, M. Meireles, A. Bouchoux, J. Sprakel, K. Schroën, Deswelling and deformation of microgels in concentrated packings, Sci. Rep. 7 (2017) 10223.

[25] A. Stradner, J.J. Crassous, K. van Gruijthuijsen, M. Obiols-Rabasa, P. Schurtenberger, P.S. Mohanty, R. Schweins, S. Nöjd, Interpenetration of polymeric microgels at ultrahigh densities, Sci. Rep. 7 (2017) 1487.

[26] G.M. Conley, P. Aebischer, S. Nöjd, P. Schurtenberger, F. Scheffold, Jamming and overpacking fuzzy microgels: deformation, interpenetration, and compression, Sci. Adv. 3 (2017) e1700969.

[27] J. Mattsson, H.M. Wyss, A. Fernandez-Nieves, K. Miyazaki, Z. Hu, D.R. Reichman, D.A. Weitz, Soft colloids make strong glasses, Nature 462 (2009) 83-86. 
[28] J. Linkhorst, T. Beckmann, D. Go, A.J.C. Kuehne, M. Wessling, Microfluidic colloid filtration, Sci. Rep. 6 (2016) 22376.

[29] S.B. Debord, L.A. Lyon, Influence of particle volume fraction on packing in responsive hydrogel colloidal crystals, J. Phys. Chem. B 107 (2003) 2927-2932.

[30] A.S.J. Iyer, L.A. Lyon, Self-healing colloidal crystals, Angew. Chem. Int. Ed. 48 (2009) 4562-4566.

[31] P. Bacchin, D. Si-Hassen, V. Starov, M.J. Clifton, P. Aimar, A unifying model for concentration polarization, gel-layer formation and particle deposition in cross-flow membrane filtration of colloidal suspensions, Chem. Eng. Sci. 57 (2002) 77-91.

[32] M. Elimelech, S. Bhattacharjee, A novel approach for modeling concentration polarization in crossflow membrane filtration based on the equivalence of osmotic pressure model and filtration theory, J. Membr. Sci. 145 (1998) 223241.

[33] R.W. Style, S.S.L. Peppin, Crust formation in drying colloidal suspensions, Proc. R. Soc. Math. Phys. Eng. Sci. 467 (2011) 174-193.

[34] A. Merlin, J.B. Salmon, J. Leng, Microfluidic-assisted growth of colloidal crystals, Soft Matter. 8 (2012) 3526-3537.

[35] C. Bonnet-Gonnet, L. Belloni, B. Cabane, Osmotic pressure of latex dispersion, Langmuir 10 (1994) 4012-4021.

[36] M. Rubinstein, R.H. Colby, A.V. Dobrynin, Dynamics of semidilute polyelectrolyte solutions, Phys. Rev. Lett. 73 (1994) 2776-2779.

[37] R.S. Koene, T. Nicolai, M. Mandel, Scaling relations for aqueous polyelectrolyte-salt solutions. 3. Osmotic pressure as a function of molar mass and ionic strength in the semidilute regime, Macromolecules 16 (1983) 231-236.

[38] A.V. Dobrynin, R.H. Colby, M. Rubinstein, Scaling theory of polyelectrolyte solutions, Macromolecules 28 (1995) 1859-1871.

[39] M. Rubinstein, R.H. Colby, A.V. Dobrynin, J.F. Joanny, Elastic modulus and equilibrium swelling of polyelectrolyte gels, Macromolecules 29 (1996) 398-406.

[40] P.J. Flory, Principles of Polymer Chemistry, Cornell University Press, Ithaca, 1969.

[41] P.J. Flory, J.J. Rehner, Statistical mechanics of cross-linked polymer networks II. Swelling, J. Chem. Phys. 11 (1943) 521-526.

[42] A. Fernandez-Nieves, A. Fernandez-Barbero, B. Vincent, F.J. Nieves, Osmotic deswelling of ionic microgel particles, J. Chem. Phys. 119 (2003) 10383-10388.

[43] D. Capriles-González, B. Sierra-Martín, A. Fernández-Nieves, A. FernándezBarbero, Coupled deswelling of multiresponse microgels, J. Phys. Chem. B 112 (2008) 12195-12200. 
[44] M. Quesada-Pérez, J.A. Maroto-Centeno, J. Forcada, R. Hidalgo-Alvarez, Gel swelling theories: the classical formalism and recent approaches, Soft Matter. 7 (2011) 10536-10547.

[45] R.G.M. van der Sman, Biopolymer gel swelling analysed with scaling laws and Flory-Rehner theory, Food Hydrocoll. 48 (2015) 94-101.

[46] A. Bouchoux, P.E. Cayemitte, J. Jardin, G. Gésan-Guiziou, B. Cabane, Casein micelle dispersions under osmotic stress, Biophys. J. 96 (2009) 693-706.

[47] R. Roa, E.K. Zholkovskiy, G. Nägele, Ultrafiltration modeling of non-ionic microgels, Soft Matter. 11 (2015) 4106-4122.

[48] M.M. Hedrick, J.K. Chung, A.R. Denton, Structure and osmotic pressure of ionic microgel dispersions, J. Chem. Phys. 142 (2015) 034904.

[49] P.S. Mohanty, D. Paloli, J.J. Crassous, E. Zaccarelli, P. Schurtenberger, Effective interactions between soft-repulsive colloids: experiments, theory, and simulations, J. Chem. Phys. 140 (2014) 094901.

[50] T.G. Mason, M.D. Lacasse, G.S. Grest, D. Levine, J. Bibette, D.A. Weitz, Osmotic pressure and viscoelastic shear moduli of concentrated emulsions, Phys. Rev. E 56 (1997) 3150-3166.

[51] P. Flodin, The sephadex story, Polym. Eng. Sci. 38 (1998) 1220-1228.

[52] R.J.H. Stenekes, O. Franssen, E.M.G. van Bommel, D.J.A. Crommelin, W.E. Hennink, The preparation of dextran microspheres in an all-aqueous system: effect of the formulation parameters on particle characteristics, Pharm. Res. 15 (1998) 557-561.

[53] R.J.H. Stenekes, O. Franssen, E.M.G. van Bommel, D.J.A. Crommelin, W.E. Hennink, The use of aqueous PEG/dextran phase separation for the preparation of dextran microspheres, Int. J. Pharm. 183 (1999) 29-32.

[54] W. Schaertl, H. Sillescu, Brownian dynamics of polydisperse colloidal hard spheres: equilibrium structures and random close packings, J. Stat. Phys. 77 (1994) 1007-1025.

[55] R.J.H. Stenekes, S.C. De Smedt, J. Demeester, G. Sun, Z. Zhang, W.E. Hennink, Pore sizes in hydrated dextran microspheres, Biomacromolecules 1 (2000) 696-703.

[56] R.J.H. Stenekes, W.E. Hennink, Equilibrium water content of microspheres based on cross-linked dextran, Int. J. Pharm. 189 (1999) 131-135.

[57] Y. Liu, R. Lipowsky, R. Dimova, Concentration dependence of the interfacial tension for aqueous two-phase polymer solutions of dextran and polyethylene glycol, Langmuir 28 (2012) 3831-3839.

[58] C.H. Kang, S.I. Sandler, Effects of polydispersivity on the phase behavior of the aqueous two-phase polymer systems, Macromolecules 21 (1988) 3088-3095. 
[59] B.G. De Geest, C. Déjugnat, M. Prevot, G.B. Sukhorukov, J. Demeester, S.C. De Smedt, Self-rupturing and hollow microcapsules prepared from biopolyelectrolytecoated microgels, Adv. Funct. Mater. 17 (2007) 531-537.

[60] A. Bouchoux, P.E. Cayemitte, J. Jardin, G. Gésan-Guiziou, B. Cabane, Casein micelle dispersions under osmotic stress, Biophys. J. 96 (2009) 2547 https://www.cell.com/ biophysj/fulltext/S0006-3495(09)00491-3.

[61] F. Cavalieri, E. Chiessi, I. Finelli, F. Natali, G. Paradossi, M.F. Telling, Water, solute, and segmental dynamics in polysaccharide hydrogels, Macromol. Biosci. 6 (2006) 579-589.

[62] C. Reid, R.P. Rand, Fits to osmotic pressure data, Biophys. J. 73 (1997) 16921694.

[63] G.R. Farrell, K.M. Martini, N. Menon, Loose packings of frictional spheres, Soft Matter 6 (2010) 2925-2930.

[64] S. Kuga, Pore size distribution analysis of gel substances by size exclusion chromatography, J. Chromatogr. A 206 (1981) 449-461.

[65] R. Podgornik, J. Hopkins, V.A. Parsegian, M. Muthukumar, Polymers pushing polymers: polymer mixtures in thermodynamic equilibrium with a pore, Macromolecules 45 (2012) 8921-8928.

[66] P.A. Gurnev, C.B. Stanley, M.A. Aksoyoglu, K. Hong, V.A. Parsegian, S.M. Bezrukov, Poly(ethylene glycol)s in semidilute regime: radius of gyration in the bulk and partitioning into a nanopore, Macromolecules 50 (2017) 2477-2483.

[67] Rand, Osmotic Pressure Data (n.d.). https://brocku.ca/researchers/ peter_rand/ osmotic/osfile.html. (Accessed 13 April 2018).

[68] T.G. Mason, J. Bibette, D.A. Weitz, Elasticity of compressed emulsions, Phys. Rev. Lett. 75 (1995) 2051-2054.

[69] S. Nezamabadi, Farhang Radjai, Julien Averseng, Jean-Yves Delenne, Implicit frictional-contact model for soft particle systems, J. Mech. Phys. Solids 83 (2015) 72-87.

[70] J.A. Cohen, S. Highsmith, An improved fit to website osmotic pressure data, Biophys. J. 73 (1997) 1689-1692.

[71] C.G. Lopez, W. Richtering, Does Flory-Rehner theory quantitatively describe the swelling of thermoresponsive microgels? Soft Matter. 13 (2017) 8271-8280.

[72] W.N.E. van Dijk-Wolthuis, J.J. Kettenes-van den Bosch, van der Kerk-van Hoof, W.E. Hennink, Reaction of dextran with glycidyl methacrylate: an unexpected transesterification, Macromolecules. 30 (1997) 3411-3413.

[73] W.N.E. van Dijk-Wolthuis, O. Franssen, H. Talsma, M.J. van Steenbergen, J.J. Kettenes-van den Bosch, W.E. Hennink, Synthesis, characterization and 
polymerization of glycidyl methacrylate derivatized dextran, Macromolecules. 28 (1995) 6317-6322.

[74] J.L. van Roon, E. Groenendijk, H. Kieft, C.G.P.H. Schroën, J. Tramper, H.H. Beeftink, Novel approach to quantify immobilized-enzyme distributions, Biotechnol. Bioeng. 89 (2005) 660-669. 



\section{Deswelling and deformation of microgels in concentrated packings}

This chapter has been published as:

I. Bouhid de Aguiar, T. van de Laar, M. Meireles, A. Bouchoux, J. Sprakel, and K. Schroën Deswelling

and deformation of microgels in concentrated packings Scientific Reports, vol. 7, no. 1, p 10223, 2017 


\section{Abstract}

Increasing the particle density of a suspension of microgel colloids above the point of random-close packing, must involve deformations of the particle to accommodate the increase in volume fraction. By contrast to the isotropic osmotic deswelling of soft particles, the particle-particle contacts give rise to a non-homogeneous pressure, raising the question if these deformations occur through homogeneous deswelling or by the formation of facets. Here we aim to answer this question through a combination of imaging of individual microgels in dense packings and a simple model to describe the balance between shape versus volume changes. We find a transition from shape changes at low pressures to volume changes at high pressures, which can be explained qualitatively with our model. Whereas contact mechanics govern at low pressures giving rise to facets, osmotic effects govern at higher pressures, which leads to a more homogeneous deswelling. Our results show that both types of deformation play a large role in highly concentrated microgel suspensions and thus must be taken into account to arrive at an accurate description of the structure, dynamics and mechanics of concentrated suspensions of soft spheres. 


\subsection{Introduction}

Microgels are colloidal particles made from a solvent-swollen crosslinked polymer network [1,2] whose softness can be tuned with the crosslink density [3]. These microgels are commonly used as a well-defined experimental model system to explore the phase behavior, dynamics and mechanics of soft particle suspensions $[4,5]$. Their softness, which entails both a low resistance to shape and to volume changes, has a large effect on the properties of dense suspensions of these particles. For example, they can be compressed to packing density in excess of random close packing and their increase in viscosity as they approach the liquid-solid boundary shows significant deviations from the behavior of hard spheres [6,7] Moreover, microgels exhibit a rich phase behavior [8-10], which can be tailored by their degree of crosslinking [11], the presence of charges [12] or inherent network inhomogeneities [13].

To achieve effective packing densities of well above the random close packing limit for hard spheres, the reduction in available volume must be accommodated by either shape or volume changes in the constituent particles. This can occur either by forming facets at the contact points with the surrounding particles $[8,14,15]$ and by the expulsion of solvent from the particle, leading to homogeneous deswelling and volume reduction [16,17]. Recent work has highlighted how the latter can have pronounced effects on the interpretation of experiments on microgels, since osmotic deswelling can lead to substantial deviations between the apparent and real particle volume fraction [18].

It is most likely that faceting and homogeneous deswelling are relevant to some extent; however, this remains relatively unexplored. Recent contrast-variation scattering experiments have shed light on this complexity for the first time, showing an interplay of deformations, deswelling and even interpenetration of surfacedangling chains as the particle concentration is varied [19]. Yet, our quantitative understanding of particle deswelling and deformation remains incomplete.

The isotropic compression of individual microgels subjected to a homogeneous osmotic force has been studied in detail previously. For example, microgels suspended in solutions of a polymeric osmolyte, such as dextran which is excluded from the microgel network, exhibit a homogeneous osmotic deswelling consistent with polymer swelling theory [6] from which the bulk modulus $K$ of the individual microgels could be determined. Squeezing a single microgel between two sapphire plates, yielding two discrete contact points, has shown that this is a controlled way of probing deformations of single particles, but the possibility of an interplay between 
shape and volume changes was not discussed [17] In this last case, the microgel is under non-homogeneous pressure. This implies that contact deformations cannot be ignored as it is also the case for shape or volume changes, dictated by the Poisson ratio, which is typically between 0.4-0.45 for hydrogel particles [20]. Of course, the same argument holds for particles with more than two contact points, as would be the case in a dense packing of particles, contacting multiple neighbors. The fact that both effects contribute to microgel shape and size in non-homogeneous pressure fields is illustrated by the capillary micromechanics work of Guo and Wyss [5] where individual soft particles are brought into a tapered confinement, which induced both shape and size changes, that can be quantified accurately, for example to derive the full linear mechanics of single particles.

So, while it is clear that the mechanical response of compressible and deformable microgels to complex pressure fields involves both shape and size changes, these effects remain to be explored in dense packings of many microgels in close contact. Understanding these effects is an important step towards a more comprehensive description of the combined effects of single-particle mechanics and osmotic equilibrium on the properties of concentrated suspensions of soft particles.

In this chapter, we explore the deformation and deswelling mechanisms of microgels in compressed microgel packings and provide a framework to understand their behavior. We osmotically stress mixtures of fluorescent and non-fluorescent microgels and image the shape and size of single microgels with high resolution using confocal microscopy and quantitative analysis algorithms. We find that the ratio of shape to volume changes, evolves non-monotonically with applied pressure; at low pressures shape changes are pronounced, in the form of facets, while at larger pressure the facets disappear and the microgels assume a spherical shape by deswelling homogeneously. We qualitatively explain these results using a simple mechanical model, which combines the osmotic pressure of the gel network with contact mechanics.

\subsection{Experimental}

\section{Microgel synthesis}

We synthesize polyacrylamide microgels by polymerization of monomer solutions in emulsion droplets as a template. In a round bottom flask, we mix $100 \mathrm{ml}$ kerosene with $1 \%$ wt of the surfactant polyglycerol polyricinoleate (PGPR90). In a separate flask we prepare our monomer solution with $10 \mathrm{ml}$ of water, $0.1 \mathrm{M}$ sodium hydroxide solution to set the $\mathrm{pH}$ at $8.5,2.5 \mathrm{~g}$ of acrylamide, $50 \mathrm{mg}$ of potassium persulfate 
(KPS) and $25 \mathrm{mg}$ of N,N'-methylenebisacrylamide (BIS) as the crosslinker at 1\%wt as compared to the total monomer content. For fluorescent microgels, we include 25 mg of fluorescein methacrylate at this stage. We add our monomer solution to the content of the round bottom flask and emulsify the aqueous phase into the oil phase under high shear with a rotor-stator mixer for three minutes. We then close the round bottom flask with a rubber septum and bubble the emulsion with nitrogen for 20 minutes to remove oxygen. We subsequently place the round bottom flask on a stirring plate on ice and we inject $1 \mathrm{ml} \mathrm{N}, \mathrm{N}, \mathrm{N}$ ',N'-tetramethylethylenediamine (TEMED) to trigger the polymerization. We allow the system to react for 2-3 hours and precipitate the microgels in cold methanol. We clean our microgels by repeated centrifugation and resuspension steps, first in methanol to remove excess kerosene and surfactant, and finally in water, after which the microgel suspension is stored at $4^{\circ} \mathrm{C}$.

\section{Osmotic stress}

We use a mixture of fluorescent and non-fluorescent microgel suspensions at a number ratio of 1:20 to allow observation of individual microgels in the packing using confocal fluorescence microscopy. We compress the microgel suspension using the osmotic stress technique. We place the suspension of microgels in dialysis bags that we then place in a solution of polyethylene glycol (PEG) with known concentration. The concentration of a PEG solution can be correlated to its osmotic pressure through empirical equations available in the literature [21]. We use a range of PEG concentrations corresponding to osmotic pressures between $2.5 \mathrm{kPa}$ and $2 \mathrm{MPa}$. The volume of dialysate is at least 100 times larger than the sample volume. The system is allowed to equilibrate for two weeks to ensure the desired compression pressure $P$ is achieved. The dialysate is renewed in the middle of this process, after one week.

\section{Confocal microscopy}

To determine how the microgels deform we use confocal fluorescence microscopy to record three-dimensional image stacks of individual, fluorescently labelled microgels. As we only have a small amount of fluorescently labelled microgels in each sample we can visualize single microgels as they deform and shrink at varying osmotic pressure. These experiments are performed on a Zeiss microscope, equipped with a $488 \mathrm{~nm}$ laser line and imaged using a x100 oil-immersion objective. The resolution of the images is $1000 \times 1000$ pixels. To measure the type and degree of deformation of a microgel at different compression pressures we analyze the confocal image stacks using custom MATLAB routines. To accurately determine the surface area and circumference of a microgel in each confocal slice we trace the boundary of every microgel and fit a polynomial function to this shape using Savitsky-Goley smoothing. 
We calculate the surface area for each slice in our three-dimensional image stack and determine the total volume of each microgel in our field of view.

\subsection{Results and discussion}

We study microgels made from poly(acrylamide) (pAAm) prepared by emulsion templating. The particles are crosslinked with $1 \%$ wt of crosslinker with respect to the total monomer content, resulting in reasonably soft microgels. Here we aim to prepare microgels with sizes larger than $10 \mu \mathrm{m}$ such that their shape and size can be carefully deduced from confocal fluorescence microscopy experiments. Although our microgels are large enough to be imaged by brightfield microscopy, it remains challenging to obtain the entire shape and size due to significant artefacts that arise due to the high particle concentration. Rather, we choose to use confocal microscopy to visualize a few labelled microgels in a large excess of undyed particles. This enables us to resolve the microgel shape and size with high resolution and without being hindered by the high particle density. To concentrate the microgel suspensions to a well-defined macroscopic osmotic pressure, we stress the suspensions by placing them in a dialysis membrane and equilibrating them against poly(ethylene glycol) (PEG) as an osmolyte, which leads to a homogeneous compression of the suspension to osmotic pressure differences between $10^{3}-10^{6} \mathrm{~Pa}$.
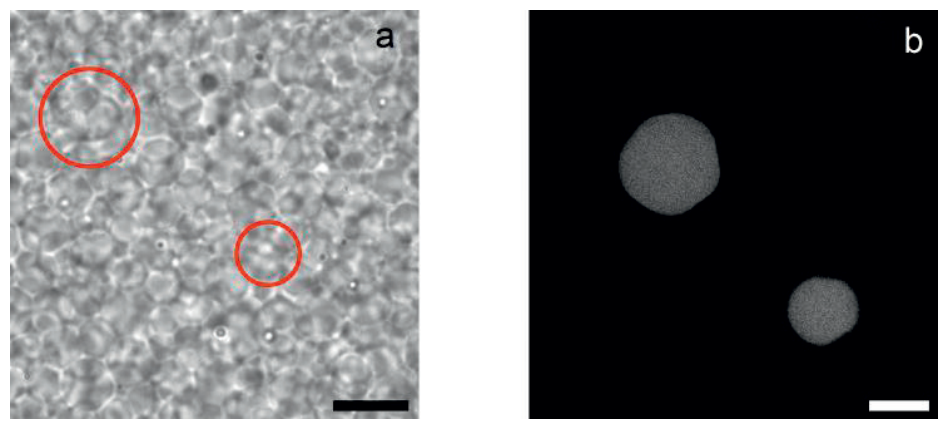

Figure 1. Compressed microgel packing composed of a mixture of fluorescent and non-fluorescent microgels. (a) Bright field image. Scale bar denotes $20 \mu \mathrm{m}$. (b) CLSM image. Scale bar denotes $10 \mu \mathrm{m}$. The images were made at the same region of the sample. Red circles in the bright field image indicate the position of the fluorescent particles but are not to scale. 

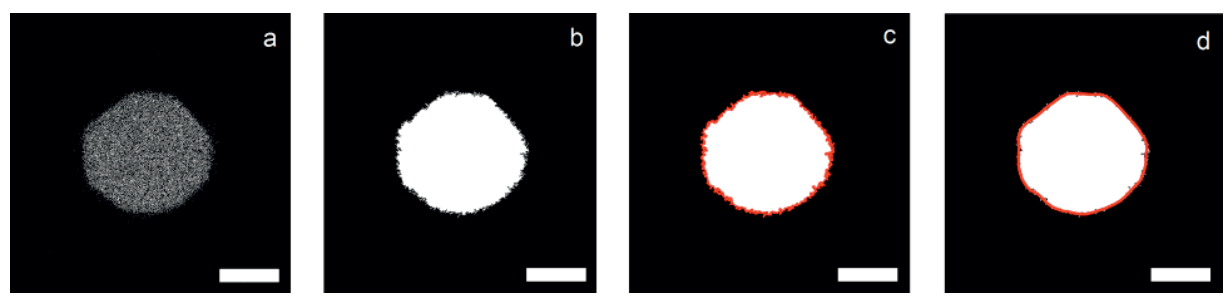

Figure 2. Steps during image treatment, (a) shows the raw microscopy data, (b) shows the binary version of this image, generated to most closely preserve the microgel shape, (c) shows a simple edge trace of this binary image, resulting in many artifacts, (d) shows the much smoother tracing after fitting this boundary with a Savitzky-Golay filter, which results in a smooth boundary while preserving the overall microgel shape. Scale bars denote $5 \mu \mathrm{m}$.

If all the microgels are fluorescently labelled, observing the boundaries of a single particle at its contacts with neighbors becomes highly inaccurate. To end this, we use a mixture of fluorescent and non-fluorescent microgels in our experiments. This allows us to accurately observe a single fluorescent microgel that is surrounded by non-fluorescent microgels. While identification of single particles is difficult in bright-field microscopy images (Figure 1a) where all particles provide contrast, welldefined images of single fluorescent particles can be made using confocal microscopy, as shown in Figure 1b.

To quantify changes in size and shape of the individual microgels in the packings, we image at least twenty separate microgels in three-dimensions using confocal microscopy for each compression pressure. From these images, we can calculate the microgel volume and shape. In order to calculate an accurate perimeter and area for each slice in the three-dimensional image stacks, we first convert our images (Figure 2a) to binary black and white (Figure 2b). During this thresholding, pixelation at the background-particle edge results in jagged edges in an edge-detection algorithm (Figure 2c). Such roughness on the perceived perimeter would overestimate the particle contour. This discretization effect can be minimized by recording highresolution confocal images, but some boundary effects remain. To solve this issue, we first trace this perimeter using a Savitsky-Golay (SG) filter [22] to smooth the boundary (Figure 2d). From these smoothed traces we then reconstruct the image, which allows us to calculate the perimeter and area much more accurately, while still having access to the overall microgel shape without blunting due to the filtering. 

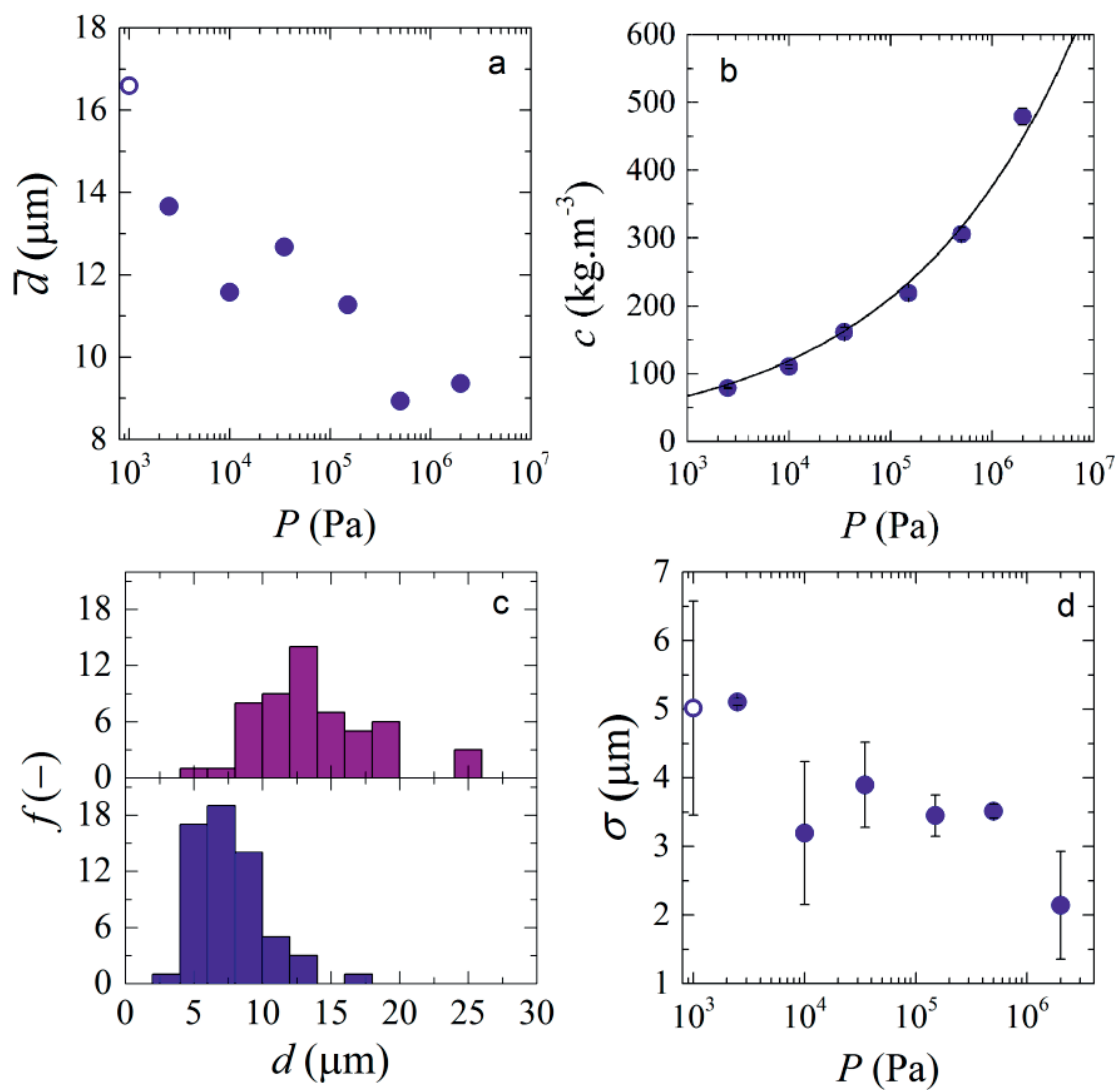

Figure 3. (a) Average microgel diameter $(\bar{d})$ as a function of compression pressure, (b) microgel packing polymer concentrations $c$ as a function of pressure $P$. Solid line describes the $P \propto c^{4}$ scaling. (c) size distributions of microgel diameter (d) for two compression pressures (2.5 $\mathrm{kPa}$ - top and $2 \mathrm{MPa}$ - bottom), which shows a narrowing of the size distribution at increased compression and (d) width of fitted Gaussian functions $(\sigma)$ for each size distribution as a function of compression pressure. Open symbols are values obtained at zero pressure $(P)$.

We first probe the changes in particle volume, to evaluate their osmotic deswelling in response to the contact pressure of neighboring particles. We deduce the particle volume from the equivalent sphere diameter $d$ fitted to the three-dimensional image stacks of thresholded and filtered images of single particles. As expected, we observe that the average size of the microgels decreases with increasing compression pressure, as a result of solvent expulsion by the microgels (Figure 3a). The average values were obtained by averaging over multiple particles in a polydisperse 

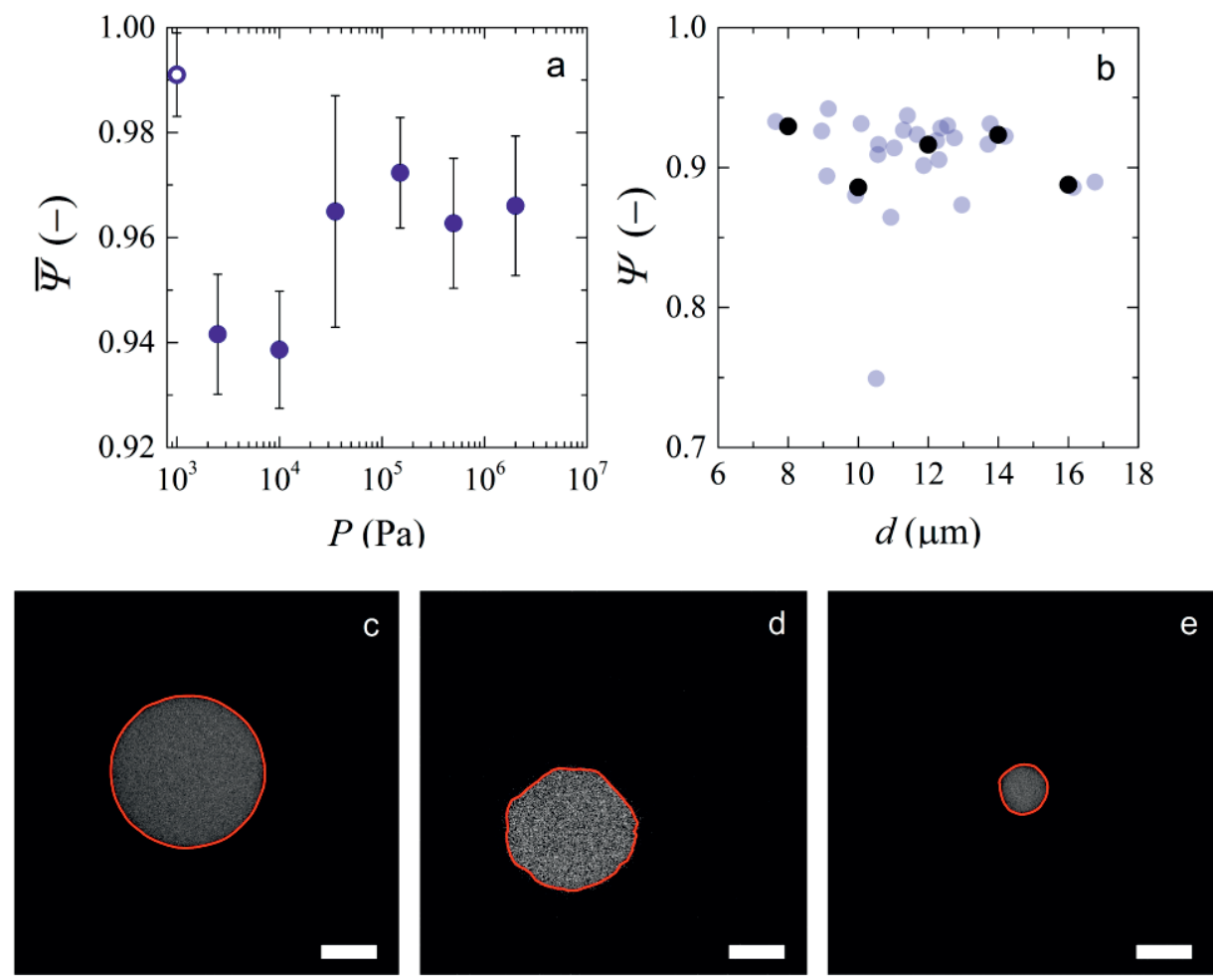

Figure 4. (a) Average sphericity $(\Psi)$ as a function of compression pressure. The open symbol is a value obtained at zero pressure $P=0$. (b) Microgel sphericity $(\Psi)$ as a function of microgel diameter $(d)$ for a single compression pressure $(10 \mathrm{kPa})$. Black circles are binned data. Bin width is 2 and the values within each bin were averaged. Confocal images of a single microgel at (c) zero pressure, (d) at $P=10 \mathrm{kPa}$ and (e) 2 $\mathrm{MPa}$. Red outlines correspond to the edges of the particle after image analysis. Scale bars denote $5 \mu \mathrm{m}$.

population; nevertheless, we see a clear monotonic trend of deswelling with increasing pressure $P$, consistent with previous reports $[17,23]$.

For each compression pressure $P$, we also determine the polymer concentration in the compressed microgel packings by dehydrating the particle pastes and measuring the dry weight (Figure 3b). We find that the osmotic pressure increases steeply with increasing polymer concentration. The data is well described by a scaling $P \propto c^{4}$, which is significantly higher than the scaling prediction for the osmotic pressure of a semi-dilute polymer solution within the blob model of $P \propto c^{9 / 4}$ [24] We attribute this to the additional contribution of network elasticity to the osmotic pressure, as 

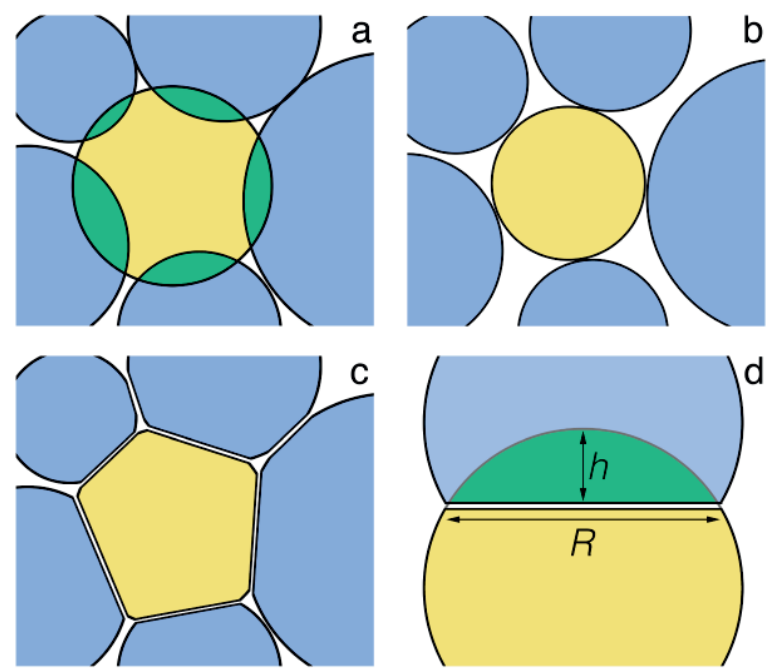

Figure 5. Osmotic compression of a microgel suspension leads to unphysical overlap between neighbors (a), which is resolved either by deswelling the particles (b) or through particle deformation in the form of contact facets (c). In the calculation of the energy of deformation by facet formation $U_{f}$ we use a Hertzian contact model in which the deformation is approximated by the overlap with penetration depth $h$ leading to facets of size $R$ (d).

described by the Flory-Rehner theory [25] where the rise in osmotic pressure with concentration, in particular close to the equilibrium swelling state of the particles, is much steeper than that of a simple solution of linear chains [26].

From our experimental observations, we do not only have access to the average particle size as a function of compression, but also the size distribution. Interestingly, we see how the size distribution shifts as the compression increases (Figure 3c). This is likely due to the fact that larger microgels will be more compressed in the packings, whereas small particles can reside in interstitial spaces and thus experience smaller contact pressures on average. As a consequence, larger particles will deswell more than smaller ones, thus narrowing the size distribution of the sample. To quantify the change in size distribution, we measure the width of the particle size distributions $\sigma$ as the full width at half maximum (FWHM) by fitting the experimental data to a normal distribution at all compression pressures. Indeed, the width of the distribution decreases with increasing pressure (Figure 3d). We also plotted (results not shown) the ratio between the gaussian width and the mean with varying pressure and obtained the same decreasing trend. The fact that we obtained the same trend shows that the decrease in size of the particles is not the reason for the narrowing of 
the size distribution. This observation of a narrowing particle size distribution is consistent with earlier reports of a co-crystallization of large microgels in a bath of smaller particles as the pressure increased, leading to shrinkage of the larger particles to fit into the microgel lattice [27].
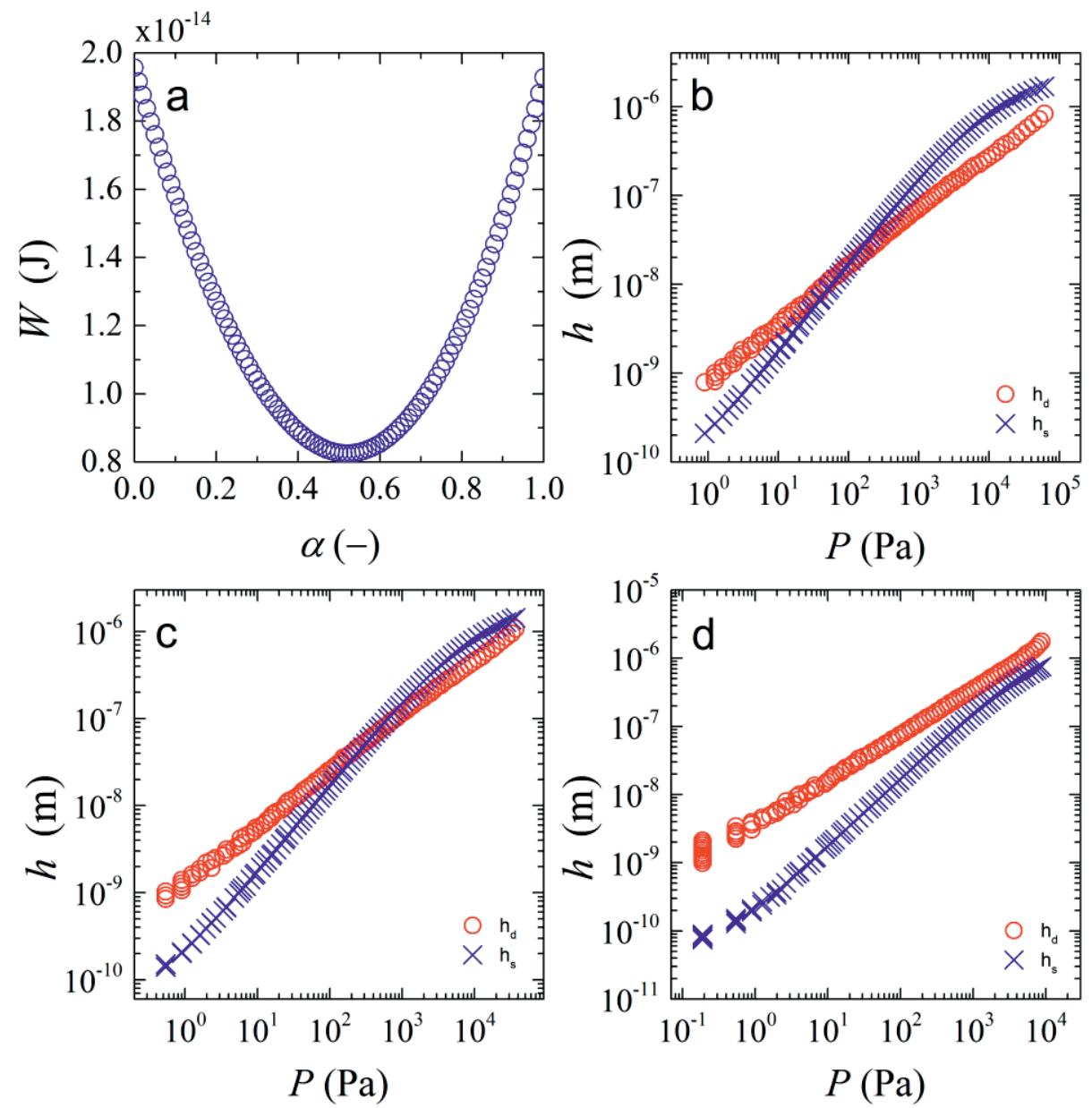

Figure 6. (a) Mechanical work $W$ as a function of the fraction of the overlap that is mitigated through deformation $\alpha=h_{d} /\left(h_{s}+h_{d}\right)$, showing a clear minimum where $d W / d \alpha=0$, signalling the equilibrium condition (for: $v=0.40$ and $h_{s}+h_{d}=$ $100 \mathrm{~nm}$ ), (b-d) Contributions of shrinkage $h_{s}$ and deformation $h_{d}$ to the total particle response as a function of the total pressure $P$ for three different values of the Poisson's ratio $v=0.3(\mathrm{~b}), 0.4(\mathrm{c})$ and $0.48(\mathrm{~d})$. 
Clearly, increasing the particle density leads to pronounced osmotic deswelling of the particles. However, visual inspection of the confocal microscopy images shows also how distinct facets develop at the particle-particle contact points (Figure 2). While most previous studies have studied in-depth the volume changes associated with osmotic compression of microgel packings, these shape changes have received much less attention so far, but may be crucial to understand the rheology and dynamics of microgel pastes.

To evaluate the extent of shape changes, we determine to what extent the particle shape deviates from a perfect sphere. Due to the preparation templated in emulsion droplets, the rest shape of the microgels is a near-perfect sphere. We define the sphericity, extracted from our two-dimensional confocal images as:

$$
\Psi=\frac{2 \sqrt{\pi N_{a}}}{N_{\text {circ }}}
$$

where $N_{a}$ is the number of pixels in the area enclosed by the SG filtered boundary (red line in Figure $2 \mathrm{~d}$ ) and $N_{\text {circ }}$ is the number of pixels along the boundary contour. For a perfect circle $\Psi \equiv 1$, while any asphericity, e.g. due to facetting, will result in $\Psi<1$.

Samples at zero pressure $P=0$, exhibit an almost perfect spherical geometry with $\Psi \approx 0.99 \pm 0.1$ (Figure 4c); the small deviation from $\Psi=1$ is caused by the inevitable discretization of the images at the scale of a pixel, which cannot be completely circumvented by the SG filtering of the particle contour.

At finite pressure, the sphericity, averaged over at least twenty particles at each pressure, initially decreases. This indicates that the microgels become deformed by the formation of facets at contact points with neighboring particles (Figure 4d). As the compression pressures $P$ increase, the average sphericity of the microgels increases again, which indicates that the particles regain their spherical shape (Figure 4e). For comparison, we evaluated images containing polygons and obtained sphericity values for a hexagon and a square of 0.97 and 0.91 , respectively. We also evaluated images of spheres of different sizes to rule out the effect of the amount of pixels constituting the sphere in the sphericity values. We found that for the size of images used (1000x1000 pixels), or the size of the sphere did not interfere in the sphericity results. Finally, to investigate whether the size polydispersity of the microgels has an influence on their degree of deformation, we plot the sphericity $\Psi$ as a function of the diameter of the microgels $d$ (Figure $4 \mathrm{~b}$ ) for a certain compression 
pressure $(10 \mathrm{kPa})$. We find no statistically significant trend, indicating that there is no significant effect of size polydispersity on the particle deformations, within the statistical noise of our experiment.

Our experimental results show that both faceting and deswelling happen, depending on the applied pressure, in a distinctly non-monotonic way. As the pressure increases, facets first become more pronounced, until they start to become less noticeable and the particle appears to homogeneously deswell to a (smaller) spherical configuration. This counterintuitive observation triggers the question if these are equilibrium effects, or whether non-equilibrium aspects may be important. First, we note that the samples are equilibrated for 14 days at a given osmotic pressure. The timescales for poroelastic relaxation, i.e. the solvent flow within the porous polymer particles required to achieve shape and size changes, occurs on much smaller time scales, and are thus not likely to contribute. Moreover, experiments conducted at different times give identical results, suggesting time-dependencies not to be of significant influence.

To confirm that the change from faceting at low pressure to osmotic deswelling at higher pressures is an equilibrium effect, we derive a simple equilibrium model that is capable of reproducing the observed behavior by balancing contact mechanics versus osmotic effects upon creating particle-particle contacts.

Since the experimental microgel particles of polyacrylamide are under good solvency conditions, we derive an extension on the classical description of Flory and Rehner $[28,29]$ which assumes ideal chains between nodes that are marginally stretched, to account for large chain extensions. The osmotic pressure within a microgel particle results from two opposing terms. The first is a mixing term, describing the mixing entropy and the enthalpy of solvent-monomer interactions, which promotes swelling. Within the mean-field Flory-Rehner approach this can be written as:

$$
\Pi_{\text {mix }}=\frac{k_{B} T}{a^{3}}\left(-\varphi-\ln (1-\varphi)-\chi \varphi^{2}\right)
$$

where $k_{B} T$ is the thermal energy, $a$ the size of a statistical chain segment, $\chi$ the Flory interaction parameter and $\varphi$ the monomer volume fraction, which is the main control parameter. This term is always positive and as such promotes the uptake of solvent within the microgel particle.

The mixing pressure is balanced by the elasticity of the chain segments between crosslinks. Swelling stretches the chains between crosslinks which reduces their conformational entropy. Traditionally, within the Flory-Rehner description, this 
entropic elasticity is estimated within the Gaussian approximation, which assumes that chains obey a Hookean force law. However, this is only valid when the distance between two crosslinks $\mathrm{x}$ is close to the relaxed dimension of the chains $R_{g}$. For strongly swollen microgels however, chain extension between nodes may be strong, where large deviations from Hookean behavior may be expected.

To capture this limit as well, we use the freely-jointed chain (FJC) model, which describes the elastic force $F$ on a polymer chain extended to length $\xi$ as:

$$
F=\frac{\beta k_{B} T}{a}
$$

in which $\beta$ is the inverse Langevin function, that can be expanded as:

$$
\beta=3\left(\frac{\xi}{N_{x} a}\right)+\frac{9}{5}\left(\frac{\xi}{N_{x} a}\right)^{3}+\frac{297}{175}\left(\frac{\xi}{N_{x} a}\right)^{5}+\cdots
$$

where $N_{x}$ is the number of statistical segments between crosslinks. In the limit of small chain extensions this returns to the Gaussian result for which the Hookean spring constant $k=k_{B} T / N_{x} a^{2}$ is valid. Particle swelling, thereby increasing $\xi$, leads to an effective elastic pressure to counteract swelling:

$$
\Pi_{e l}=\frac{F}{\xi^{2}}=\frac{\beta k_{B} T}{a \xi^{2}}=\frac{k_{B} T}{a}\left(\frac{3}{\xi N_{x} a}+\frac{9 \xi}{5\left(N_{x} a\right)^{3}}+\frac{297 \xi^{3}}{175\left(N_{x} a\right)^{5}}+\cdots\right)
$$

where the monomer volume fraction is related to the characteristic mesh size as $\varphi=$ $N_{x} a^{3} / \xi^{3}$.

As the microgel is dissolved in a solvent, thermodynamic equilibrium requires the balancing of the pressure within the particle $\Pi_{i n}$, by swelling or deswelling, with the external osmotic pressure $\Pi_{e x}$ :

$$
\Pi_{\text {in }}=\Pi_{\text {mix }}-\Pi_{e l}=\Pi_{e x}
$$

We define the relaxed reference state of the microgel as $\Pi_{i n}=\Pi_{e x}=0$, where the polymer volume fraction within the particles $\varphi=\varphi_{0}$. The bulk modulus $K$ is defined as:

$$
K=\varphi \frac{d \Pi_{i n}}{d \varphi}
$$


The resistance of the same particle against shape changing deformations, typically by the formation of facets at the contacts of a particle with its neighbors, can be defined by its Young's modulus $E$, defined as:

$$
E=3 K(1-2 v)
$$

with $v$ the Poisson's ratio of the hydrogel particles. More comprehensive micromechanical mean-field approaches to explore the effect of particle elasticity and compressibility on microgel glasses both at rest and under shear have been reported recently $[18,30]$.

Upon increasing the pressure of a microgel suspension, physical overlap between the particles (Figure 5a) must be avoided, either by shrinking or the formation of facets (Figure 5b,c). To evaluate the extent of both of these modes of response to compression we consider the work of deformation due to faceting $W_{d}$ and the work of shrinkage $W_{S}$; both of these represent the reversible (thermodynamic) work performed on a central particle at a given number of particles, total volume of the system and temperature.

The overlap $h$ between two neighboring particles is defined in Figure 5d, which can be resolved by a linear combination of contributions due to deformation $h_{d}$ and shrinking $h_{s}: h=h_{d}+h_{s}$. The fraction of the response attributed to faceting-type deformations can thus be formulated as: $\alpha_{d}=\frac{h_{d}}{h_{d}+h_{s}}$, and the fraction contributed to shrinking as $\left(1-\alpha_{d}\right)$.

The work of shrinkage is given by:

$$
W_{S}=\Pi_{i n} \Delta V=\frac{4 \pi}{3} \Pi_{\text {in }}\left(R_{0}{ }^{3}-\left(R_{0}-h_{s}\right)^{3}\right)
$$

with $R_{0}$ the radius of the microgel in dilute conditions, where $\Pi_{\text {in }}=0$.

The work of deformation by forming facets is gauged by using the Hertzian model for the elastic contact between two spheres of equal size $R_{0}$. The force required to form an indentation of depth $h$ is given by:

$$
F=\frac{4}{3} E R_{0}{ }^{1 / 2} h^{3 / 2}
$$


such that the work required to perform a deformation of depth $h_{d}$ between two spheres becomes:

$$
W_{d}=\int_{0}^{h_{d}} F(h) d h=\frac{8}{15} E R_{0}{ }^{1 / 2} h_{d}{ }^{5 / 2}
$$

Since each microgel particles has $Z$ neighbours, the total work associated with deformations becomes:

$$
W_{d}=\frac{8}{15} E R_{0}{ }^{1 / 2} h_{d}{ }^{5 / 2} Z
$$

The total mechanical work can now be defined as:

$$
W=\alpha_{d} W_{d}+\left(1-\alpha_{d}\right) W_{s}
$$

These two contributions need to be balanced to minimize the overall mechanical work. Thus, to find the relative amounts of deformation and shrinkage, we must solve:

$$
\frac{d W}{d \alpha}=0
$$

This allows us to evaluate for each pressure, given our expressions for the microgel elasticity and the mechanical work upon compression to what extent a particle will deform and shrink, as expressed by the parameter $\alpha_{d}$. If $\alpha_{d} \approx 1$, the particle will solely deform and shrinkage is negligible; by contrast if $\alpha_{d} \approx 0$, only isotropic shrinkage occurs while the particles maintain their spherical shape.

To compute the elastic properties of the microgels, we need to choose values for the three independent parameters which govern the microgel properties: i) $N$ : the number of statistical segments between crosslinks, for which we use $N=250$ (note that the behaviour we observe is robust to the choice of the crosslinking density and is mostly sensitive to the Poisson ratio of the hydrogel). ii) $a$ : the size of a statistical unit, for polyacrylamide microgels as the experimental example, we use the Kuhn length of polyacrylamide as $a \sim 0.4 \mathrm{~nm}$ [31], iii) $\chi$ : the Flory interaction parameter describing the interactions between polymer and solvent, for polyacrylamide in water at room temperature $\chi=0.48$ [32] For the equilibrium particle size at rest $R_{0}$ we take $5 \mu \mathrm{m}$ as also used in our experiments. Even though the coordination number is known to vary with particle concentration [33] for the sake of simplicity we assume 
$Z=12$, corresponding to the close-packed limit for monodisperse spheres; also, here we find that the results are robust to the choice of $Z$.

Indeed, we see that the mechanical work $W$ has a minimum when plotted as a function of a, the fraction of the overlap $h$ mitigated by means of faceting (Figure 6a). By finding this minimum, we can now assess the relative contributions of shrinkage and deformation as a function of the applied pressure.

Interestingly, the experimental behavior is qualitatively reproduced within our approximate theory. When we plot the amount of overlap $h$ that is mitigated by faceting $h_{d}$ and shrinking $h_{s}$ as a function of the overall pressure $\Pi$, we see a crossover in the response. At low pressures, $h_{d}$ is dominant, indicating a response governed by faceting (Figure 6b-d). As the pressure is increased, we see a transition in the behavior, as a crossing point of $h_{s}$ and $h_{d}$, to a response governed by isotropic shrinkage. This is in qualitative agreement with our experimental observations. These results can seem counterintuitive from the point of view of classical contact mechanics, where the contact between two spheres would always increase its facets if they are more compressed. However, here we are dealing with a particle compressed and surrounded by other particles. As such, both contact mechanics and osmotic effects come into play.

The crossover pressure at which $h_{s}>h_{d}$ thus signals the point at which the osmotic effects of the particle suspension as a whole begin to dominate over the contact mechanics at the particle-particle contacts. At pressures beyond this crossover point, the high osmotic pressure of the particle 'bath' leads to a homogeneous deswelling of the particles.

Within the model we have chosen here, the ratio of shrinkage versus deformation depends strongly on the Poisson ratio of the microgels. For low Poisson's ratios, indicative of compressible solids, shrinkage begins to dominate at relatively low pressures. By contrast, when we choose a high Poisson's ratio, close to that for an incompressible solid, a response governed by deformation is observed. In fact, in the limit of $v \rightarrow 0.5, h_{s}$ goes to zero and $h \approx h_{d}$.

Experimentally, we find a crossover in the sphericity at approximately $10 \mathrm{kPa}$. While the model is approximate, e.g. by the choice of a mean-field approach for the osmotic pressure and ignoring the molecular details of the particle surface, comparing this value to the theoretical cross-over pressures, indicates that the Poisson ratio of our experimental system is between 0.43-0.45. This is in the correct order of magnitude 
for swollen polyacrylamide hydrogels, for which $v=0.457$, as determined independently previously [34].

At this time, the agreement between theory and experiments is qualitative, since the exact equation of state is not known for these particles. While the Flory-Rehner form (Eqs. (2-(5) is a common starting point, it does not take microscopic details, such as crosslinking inhomogeneities, the effects of charges, etc. into account. It may be expected that changing the exact nature of the equation of state, or of the expressions used to related the network structure to the shear rigidity, will change the crossover pressure at which osmotic effects begin to govern over contact mechanics. However, the general notion that at low overall osmotic pressure the particle-particle contacts themselves dominate the particle deformation, while the bath pressure takes over when it becomes sufficiently large, is expected to hold irrespective of the choices for the equation of state. In fact, since the faceting is most sensitive to the Young's modulus of the particles, while homogeneous deswelling is governed by their bulk modulus, we may speculate that the Poisson ratio of the particle is the governing metric for if and when a crossover in behavior may be expected.

Depending on the manner in which microgels are prepared, the surface structure of the polymer chains may be different, leading to significant variations in the length and grafting density of the dangling surface polymers. Also, this can have an effect that is currently not accounted for, e.g. by the creation or suppression of lubrication layers and the establishment of a significant disjoining pressure to break these layers during compression.

Finally, recent work from our group has suggested an approach to treat the real volume fraction in systems of compressible colloids but taking osmotic deswelling into account [18], where we assumed that only deswelling occurs while faceting was presumed to be negligible. The results in the current study highlight that this approximation fails especially close to the jamming transition where faceting is severe. Interestingly, since faceting does not lead to a reduction in the real particle volume fraction with compression while deswelling does, the crossover in behavior we find indicates an even steeper effect of compression on the real versus apparent volume fraction than that predicted previously [18]. Moreover, the purpose of the previous study was to explore the effect of osmotic deswelling in absence of facets, on the slowing down of structural relaxations in microgel glasses. We may expect that facets, and the lubrication layers between the two interfaces across a facet, could alter the diffusion rate of particles with respect to their neighbors, and thus have a pronounced effect on the nature of the colloidal glass transition. In principle, this 
could be tested by comparing the behavior of particles with identical stiffness but different Poisson ratios, through which the balance between osmotic versus contact effect can be tuned.

\subsection{Conclusions}

In this chapter, we investigated the behavior of individual microgels in microgel packings under compression considering simultaneous deswelling and deformation mechanisms. Our experiments show that microgels initially facet under compression and that at higher compression pressures, they regain their spherical shape. To explain this behavior, we propose a model that balances the work of osmotic deswelling, within the Flory-Rehner picture of gel swelling, versus facet formation in the Herzian contact model. Numerical solutions of the model predict behavior qualitatively consistent with our experimental observations with a crossover from contact mechanics dominated response at low pressures to an osmotically governed response at high pressures. These results imply that treatments of the dynamics and mechanics of packings of soft particles, that account only for faceting or deswelling, are approximate, and that a full description requires taking both effects into account. This is particularly important at low pressures, close to the jamming and/or glass transition, where deformations are significant. These results also have important implications for the flow behavior of soft particles, e.g. in complex geometries such as membrane pores or constrictions [35-38] where deswelling and/or deformation plays an important role in pore passage and mitigation of clogs. 


\section{References}

[1] A. Fernández-Nieves, A. Fernández-Barbero, B. Vincent, F.J. De Las Nieves, Charge controlled swelling of microgel particles, Macromolecules 33 (2000) 2114-2118.

[2] K. Han, R. Tiwari, T. Heuser, A. Walther, Simple platform method for the synthesis of densely functionalized microgels by modification of active ester latex particles, Macromol. Rapid Comm. 37 (2016) 1323-1330.

[3] M. Destribats, V. Lapeyre, M. Wolfs, E. Sellier, F. Leal-Calderon, V. Ravaine, V. Schmitt, Soft microgels as Pickering emulsion stabilisers: role of particle deformability, Soft Matter 7 (2011) 7689-7698.

[4] B. Sung, C. Kim, M.H. Kim, Biodegradable colloidal microgels with tunable thermosensitive volume phase transitions for controllable drug delivery, J. Colloid Interf. Sci. 450 (2015) 26-33.

[5] M. Guo, H.M. Wyss, Micromechanics of soft particles, Macromol. Mater. Eng. 296 (2011) 223-229.

[6] B. Sierra-Martin, J.A. Frederick, Y. Laporte, G. Markou, J.J. Lietor-Santos, A. Fernandez-Nieves, Determination of the bulk modulus of microgel particles. Colloid Polym. Sci. 289 (2011) 721-728.

[7] J. Mattsson, H.M. Wyss, A. Fernandez-Nieves, K. Miyazaki, Z. Hu, D.R. Reichmann, D.A. Weitz, Soft colloids make strong glasses, Nature 462 (2009) 83-86.

[8] S.P. Meeker, R.T. Bonnecaze, M. Cloitre, Slip and flow in soft particle pastes, Phys. Rev. Lett. 92 (2004) 198302-1.

[9] F. Di Lorenzo, S Seifert, Particulate and continuum mechanics of microgel pastes: Effect and non-effect of compositional heterogeneity, Colloid Polym. Sci. 291 (2013) 2927-2933.

[10] A. Habicht, W. Schmolke, G. Goerigk, F. Lange, K. Saalwächter, M. Ballauff, S. Seiffert,. Critical fluctuations and static inhomogeneities in polymer gel volume phase transitions. J. Polym. Sci. Pol. Phys. 53 (2015) 1112-1122.

[11] C.M. Nolan, C.D. Reyes, J.D. Debord, A.J. García, L.A. Lyon, Phase transition behavior, protein adsorption, and cell adhesion resistance of poly(ethylene glycol) cross-linked microgel particles, Biomacromolecules 6 (2005) 2032-2039.

[12] W. Su, M. Yang, K. Zhao, T. Ngai, Influence of charged groups on the structure of microgel and volume phase transition by dielectric analysis, Macromolecules 49 (2016) 7997-8008.

[13] A. Habicht, W. Schmolke, F. Lange, K. Saalwächter, S. Seiffert, The non-effect of polymer- network inhomogeneities in microgel volume phase transitions: 
Support for the mean-field perspective, Macromol. Chem. Phys. 215 (2014) 1116-1133.

[14] L. Nyström, R. Álvarez-Asencio, G. Frenning, B.R. Saunders, M.W. Rutland, M. Malmsten, Electrostatic swelling transitions in surface-bound microgels, ACS Appl. Mater. Inter. 8, (2016) 27129-27139.

[15] J. Maldonado-Valderrama, T. del Castillo-Santaella, I. Adroher-Benítez, A. Moncho-Jordá, A. Martín-Molina, Thermoresponsive microgels at the airwater interface: the impact of the swelling state on interfacial conformation, Soft Matter 13 (2017) 230-238.

[16] C. Pellet, M. Cloitre, The glass and jamming transitions of soft polyelectrolyte microgel suspensions, Soft Matter 12 (2016) 3710-3720.

[17] J.J. Lietor-Santos, B. Sierra-Martin, R. Vavrin, Z. Hu, U. Gasser, A. FernandezNieves, Deswelling microgel particles using hydrostatic pressure, Macromolecules 42 (2009) 6225-6230.

[18] P. van der Scheer, T. van de Laar, J. van der Gucht, D. Vlassopoulos, J. Sprakel, Fragility and strength in nanoparticle glasses. ACS Nano 11 (2017) 6755-6763.

[19] P.S. Mohanty, S. Nöyd, K. van Gruijthuijsen, J.J. Crassous, M. Obiols-Rabasa, R. Schweins, A. Stradner, P. Schurtenberger, Interpenetration of polymeric microgels at ultrahigh densities, Scientifc Reports 7 (2017) 1487.

[20] S.M. Hashmi, E.R. Dufresne, Mechanical properties of individual microgel particles through the deswelling transition, Soft Matter 5 (2009) 3682-3688.

[21] A. Bouchoux, P.-E. Cayemitte, J. Jardin, G. Gésan-Guiziou, B. Cabane, Casein micelle dispersions under osmotic stress, Biophys. J. 96 (2009) 693-706.

[22] A. Savitzky, M.J.E. Golay, Smoothing and differentiation of data by simplified least squares procedures, Anal. Chem. 36, 1627-1639 (1964).

[23] J.J. Liétor-Santos, B. Sierra-Martín, A. Fernández-Nieves, Bulk and shear moduli of compressed microgel suspensions, Phys. Rev. E Stat. Nonlin. Soft Matter Phys. 84 (2011) 1-4.

[24] P. de Gennes, Scaling concepts in polymer physics (Cornell University Press, 1979).

[25] P. Flory, Principles of polymer chemistry. Baker lectures 1948 (Cornell University Press, 1953).

[26] P. Menut, S. Seiffert, J. Sprakel, D.A. Weitz, Does size matter? Elasticity of compressed suspensions of colloidal- and granularscale microgels. Soft Matter 8 (2012) 156-164.

[27] A. Scotti, U. Gasser, E.S. Herman, M. Pelaez-Fernandez, J. Han, A. Menzel, L.A. Lyon, A. Fernandez-Nieves, The role of ions in the self-healing behavior of soft particle suspensions, P. Natl. Acad. Sci.113 (2016) 5576-5581. 
[28] P.J. Flory, J. Rehner, Statistical mechanics of cross-linked polymer networks I. Rubberlike elasticity, J. Chem. Phys. 11 (1943) 512.

[29] P.J. Flory, J. Rehner, Statistical mechanics of cross-linked polymer networks II. Swelling. J. Chem. Physs 11 (1943) 521-526.

[30] J.R. Seth, L. Mohan, C. Locatelli-Champagne, M. Cloitre, R.T.A. Bonnecaze, Micromechanical model to predict the flow of soft particle glasses, Nat. Mater. 10 (2011) 838-843.

[31] S. Kundu, A.J. Crosby, Cavitation and fracture behavior of polyacrylamide hydrogels. Soft Matter 5 (2009) 3963-3968.

[32] N. Kayaman, O. Okay, B.M. Baysal, Swelling of polyacrylamide gels in polyacrylamide solutions, J. Polym. Sci. Pol. Phys. 36 (1998) 1313-1320.

[33] M. van Hecke, Jamming of soft particles: geometry, mechanics, scaling and isostaticity, J. Phys-Condens. Mat. 22 (2010) 33101.

[34] T. Takigawa, Y. Morino, K. Urayama, T. Masuda, Osmotic Poisson's ratio and equilibrium stress of poly(acrylamide) gels, Polym. J. 28 (1996) 1012-1013.

[35] J. Linkhorst, T. Beckmann, D. Go, A. Kuehne, M. Wessling, Microfluidic colloid filtration, Sci. Rep. 6 (2016) 22376.

[36] G. Hendrickson, L.A. Lyon, Microgel translocation through pores under confinement. Angew. Chem. Int. Edit. 49 (2010) 2193-2197.

[37] O. Nir, T. Trieu, S. Bannwarth, M. Wessling, Microfiltration of deformable microgels. Soft Matter 12 (2016) 6512-6517.

[38] R. Roa, E.K. Zholkovskiy, G. Nagele, Ultrafiltration modeling of non-ionic microgels. Soft Matter 11 (2015) 4106-4122. 


\section{4}

\section{Microfluidic model systems used to emulate processes occurring during soft particle filtration}




\section{Abstract}

Cake layer formation in membrane processes is an inevitable phenomenon and knowing how to manage it during the filtration processes is essential. For hard particles, especially cake porosity and thickness determine the membrane flux, but when the particles forming the cake are soft the variables one has to take into account in the prediction of cake behavior increase considerably. In this work we investigate the behavior of soft polyacrylamide microgels in microfluidic model membranes through optical microscopy for in situ observation under regular flow and under enhanced gravity conditions.

Particles considerably larger than the pore are able to pass through a pore due to deformation and deswelling. We find that membrane clogging time and subsequent cake formation is not dependent on the applied pressure but rather on particle and membrane pore properties. Furthermore, we found that particle deposits subjected to low pressures and low $\mathrm{g}$ forces deform in a totally reversible fashion. Particle deposits subjected to higher pressures only deform reversibly if they can re-swell due to capillary forces, otherwise irreversible compression is observed.

For membrane processes this implies that when using deformable particles, the pore size is not a good indicator for membrane performance, and cake formation can have much more severe consequences compared to hard particles due to the sometimesirreversible nature of soft particle compression. 


\subsection{Introduction}

Soft particles are in general termed deformable when assuming different shapes upon a stimulus, and compressible when expelling solvent in response to an external force [1,2]. Although these effects are studied, they are often disregarded in technical process designs that revolve around the 'particle size'. Membrane separation is a process in which disregarding soft particles deformation and compression can have major effects, since particles larger than the pore size may end up in the permeate.

In membrane filtration processes, many types of soft particles can be present such as proteins [3,4], cells [5] and sludge flocs [6,7]. Although there are different types of membrane separation processes, ranging from microfiltration to reversed osmosis, and even gas separation, in most of these processes, particle properties play an important role. This can be either in relation to the pore size that Particles may or may not be able to pass through a pore due to the pore size, or due to the formation of an accumulation layer on top of the membrane leading to increased resistance against mass transfer, and influencing solute transmission.

It is often assumed that particles smaller than the pore size are able to pass while particles that are larger are retained [8]. Please note that this reasoning holds for membranes with uniform pores; in reality, most membranes have a pore size distribution, which makes the situation even more complex [9]. Furthermore, the previously described gate-keeper effect of pores holds for hard particles. Many particles are compressible and deformable, even to such an extent that depending on the applied driving force, they can be pushed through pores that are smaller than the original particle size [10]. This phenomenon has also been observed in emulsion filtration in which deformable liquid droplets were able to pass membrane pores that were considerably smaller $[11,12]$.

This has various implications for membrane process design. If the soft particle is to be concentrated, it is desired that the permeate is free of them, but with soft particles this is not guaranteed based on their original size [10]. If the purity of the permeate is not a problem and the presence of soft particles can be tolerated, even then their presence can be a problem if e.g. backwashing is used. During backwashing the flux direction is reversed from permeate to feed side to remove particle deposits from the top of the membrane [13,14], but this can also lead to particles being strongly pushed into the membrane, and consequently clogging it. It is clear that the link between membrane pore size or molecular weight cutoff of the filtered component is not that straight-forward to make, and in practice a lot of trial and error is involved $[15,16]$. The reason for this could be deformability of particles, and that is why we have made 
this the focal point of this study, investigating effects inside the membrane as well as on top of it in the so-called cake layer.

Although studies have been done to obtain information on cake formation in filtration, the great majority assess cake formation (particle accumulation on top of the membrane) in an indirect manner, by measuring changes in flux, and sometimes conductivity $[17,18]$. These studies generate important information for the overall process but lead not necessarily to mechanistic understanding of local behavior. Further, a number of in situ observation techniques have been developed mostly focusing on surface visualizations. We specifically mention Fane et al. who developed Optical Coherence Tomography Imaging for visualization of fouling layers as function of time, which has led to new insights in regard to deposition of oil droplets $[19,20]$. When considering processes occurring inside a membrane, to the best of our knowledge, no techniques are available.

In the last years, microfluidic devices have been used to observe the behavior of particles in systems simulating membrane filtration [21-23]. Van Zwieten et al. used them to elucidate the behavior of hard particles [24]; soft particle deposition and transmission is still an important and missing element. In this chapter, we use microfluidic methods to observe soft particle deposition and cake behavior for particles that are 'larger' than the pore size. The microfluidic devices are composed of an array of parallel channels and we use pAAm microgels as soft particles. We use constant pressure until a cake is observed on top of the channels. Clogging time, clog existence time and cake formation kinetics are measured, as is the cake volume as function of pressure. We also use a microfluidic device mounted on a high-speed centrifuge to emulate the pressure effects. We find that the clogging time is rather independent of applied pressure due to a combined effect of increased number of particles at high flux, and the higher pressure facilitating particle deformation/compression. The cake volume reduction as function of pressure is reversible as long as the particles are not too deformed due to applied g-forces.

\subsection{Material and methods Microgel synthesis and characterization}

For the microfluidic filtration experiments, we use tailor-made micrometer-sized polyacrylamide (pAAm) microgels synthesized by us. The microgels are produced by emulsion templating as described in detail in previous work [25]. The particle sizes range from $3 \mu \mathrm{m}$ to $31 \mu \mathrm{m}$ with a Sauter mean diameter (D[3,2]) of $10 \mu \mathrm{m}$. Commercially available Sephadex G100 microgels were used in some of the 
centrifugation experiments; sizes range from $35 \mu \mathrm{m}$ to $100 \mu \mathrm{m}$ with a $\mathrm{D}[3,2]$ of 64 $\mu \mathrm{m}$. Size distributions were measured by laser diffraction (Malvern Mastersizer 3000).

\section{Microfluidic devices}

The microfluidic device used for the filtration experiments was made by soft lithography. The device is composed of 30 parallel channels and each channel has 19 constrictions along its length (Figure 1). The channels have 5 different entrance angles ( 6 channels for each angle) varying from $0^{\circ}$ to $55^{\circ}$ [26]; Figure 2 shows the different angles and the dimensions of the channel constrictions.

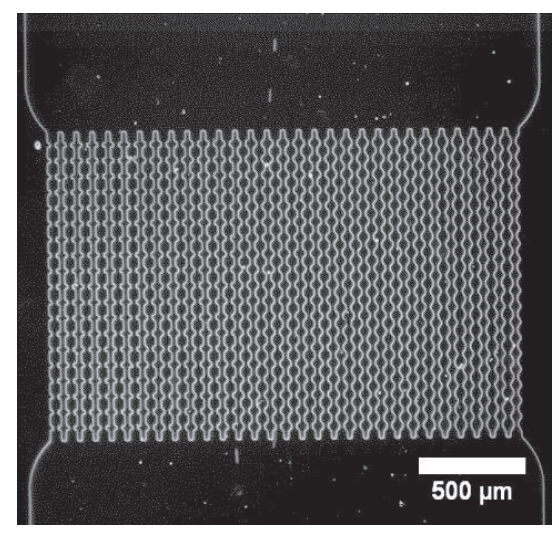

Figure 1. Optical microscopy image of the array of channels that compose the microfluid device at $2.5 \mathrm{x}$ magnification. There are 30 parallel channels with five different entrance angles. From left to right: $0,20,35,45,55^{\circ}$ (see Figure 2A).
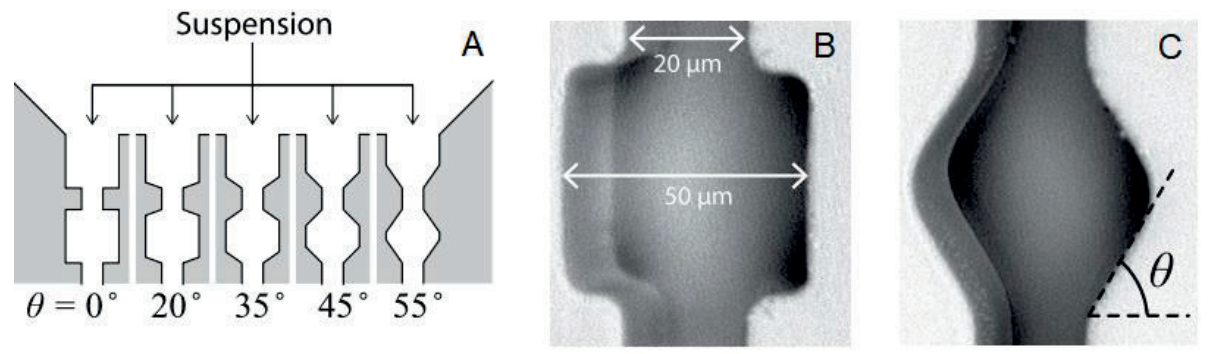

Figure 2. a) Schematic representation of the channels and their different entrance angles, b) internal dimensions of the constrictions, channel depth is $40 \mu \mathrm{m}, \mathrm{c}$ ) constriction representing the location where the angles are measured. Reproduced with permission from Nature (van de Laar et al. [26]). 
The channels are positioned in a dead-end configuration and the device has an inlet and an outlet. The devices are connected via Teflon tubing to a pressure controller (Elveflow OB1-MK3) and placed under an optical microscope. Bright field and phase contrast configurations were used. For the filtration experiments we flow a suspension of microgels of approximately $0.1 \% \mathrm{vol}$ in water at three different pressures; 50, 100 and 150 mbar with precision of 0.1 mbar. Flow of microgel suspension continues until all the channels are clogged; images are taken at a frame rate of $4 \mathrm{fps}$. After all channels are clogged, we keep the flow of microgel suspension to form a cake, or in other words, microgels accumulate on top of the channels. When a cake is formed, we vary the applied pressure to observe compression and relaxation of the cake.

\section{Centrifugation}

We also centrifuged the microgel suspension while in a microfluidic device (see Figure 3) by using a microcentrifuge coupled with an optical microscope, as described in previous work by Krebs et al. [27]. Images are taken with a highspeed camera once per rotation and the images are analyzed to determine the (compression/deformation) behavior of the microgels during centrifugation. The centrifugation speed applied varied from $12 \mathrm{~g}$ to $1058 \mathrm{~g}$. After rotation stops, images are taken to assess relaxation of the deposited microgels. Two different chamber designs were used (Figure 3).
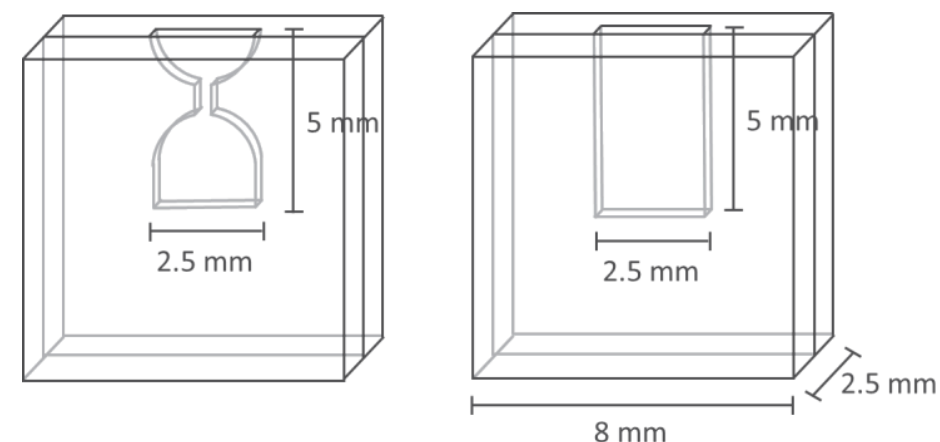

Figure 3. Microcentrifuge microchip designs. Thickness of the chambers is $100 \mu \mathrm{m}$.

For easier comparison between the centrifugation and the microfluidic filtration results, we calculated the approximate pressure at the bottom of the centrifugation chamber for each centrifugation speed used. We first calculated the approximate mass of the microgels from their density and chamber volume (considering a volume fraction of 1) and used this value to calculate the force. We then calculated the 
pressure by dividing the force by the area of the bottom of the chamber as can be seen in equation $(1$.

$$
P=\frac{V \rho g n_{g}}{A}
$$

where $P$ is the pressure at the bottom of the microchip chamber, $V$ is the volume of the microgel deposit, $\rho$ is the density of the microgels, $g$ is gravitational constant, $n_{g}$ is the number of $\mathrm{g}$ in a determined situation and $A$ is the area of the bottom of the microchip chamber.

\section{Image analysis}

The images from both microfluidics and microcentrifuge experiments are treated with home-made MATLAB scripts. For the clogging experiments, we manually select the area of the constriction where the clog happens in each channel. The code then tells us at which frame of the stack of images, the selected area is filled with microgels. The code uses black and white pixel differentiation to perform the task. For the centrifuge experiments, we also count black and white pixels, where black pixels represent the microgels and white pixels represent the background.

Throughout the chapter, we will analyze the images of the microgel deposits considering only the $2 \mathrm{D}$ projection of them. For this reason, we will refer in the next sections to cake and deposit areas and not volume. For the cake relaxation experiments, the cake area is measured manually with the help of the software ImageJ [28].

\subsection{Results and discussion System clogging time}

Microfluidic devices are used to study the behavior of microgels suspension in filtration systems. In our case, the microgels in suspension are larger than the pore constrictions but are able to modify their conformation to go through the pores. When the microgel suspension flows through the microfluidic device, the pores get blocked one by one by individual particles until the whole system is blocked. We consider the system blocked when there is at least one microgel stuck in every single microchannel. The complete system clogging time was measured as function of the applied pressure. Please note that the device clogging time was taken as the moment at which all channels contained at least one trapped microgel (Figure 4), although this does not mean that the channels were permanently clogged (see section clog stability). 


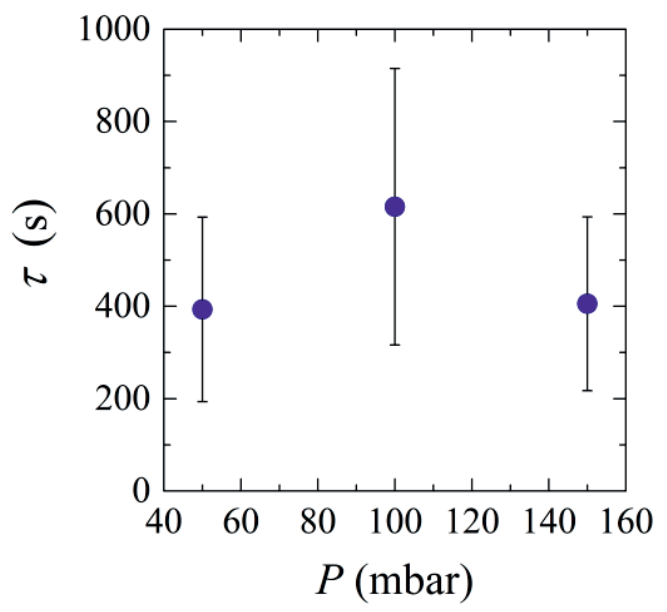

Figure 4. System clog time $(\tau)$ as a function of pressure $(P)$.

If the clogging time would be a function of the number of particles of given size and deformability passing a constriction, we would expect a decrease in clogging time with applied pressure (increasing overall flux leads to higher supply of particles). When observing the results presented in Figure 4, this is clearly not the case. At 50 mbar, the majority of the channels clog at the first constriction (results not shown), so the particles are not pushed through the pore constrictions and clog the channels as soon as they reach them. At 100 mbar, more particles are pushed into the channel and go all the way through the channels, either not blocking them, or blocking deeper in the pore (results not shown). Due to a higher flux at 150 mbar, it is more likely that bigger particles will arrive at the entrance of the channels due to the higher flux, and the propensity to clog increases because these particles are less likely to be pushed all the way through the pores due to their larger size. The large error bars are not a result of unstable clogs but a result of the particle size distribution in combination with process conditions that dictate the deformation behavior, and is linked to clogging probability.

As the channels in the microfluidic devices have a variety of entrance angles, we verified if the channels entrance angles had an impact on the channel's clogging time and found that this is not the case as expected for hard particles smaller than the constriction [25]. An influence would be expected when particles are closer to the channel/pore size and the angles may facilitate deformation, which is apparently not the case, or when clusters of small particles are responsible for clogging [26]. To be complete, when using these devices in earlier work, we found a very weak 
dependency of clogs being formed at a neighboring pore [24]; therefore, we expect this not to have played a significant role in the current work.

To understand the behavior of microgels in filtration systems in greater detail, we focused on clog existence time and cake behavior.

\section{Clog stability}

As is clear from the previous section, it is not uncommon for microgels to be pushed all the way through the pores. Sometimes, a pore is blocked but after some time the clog is released, leaving the channel open until another microgel clogs the pore again. In the experiments carried out at different pressures we observed that most of the clogs are stable, meaning that a clog formed will remain in the channel and at the same position until the end of the experiment. The percentage of clogs that are pushed through is dependent on the applied system pressure (Figure 5) and is as high as $25 \%$ at the highest applied pressure. The percentage is calculated by dividing the number of clogs that were released by the total number of observed clogs and these values are not dependent on the duration of the filtration run (5-10 minutes).

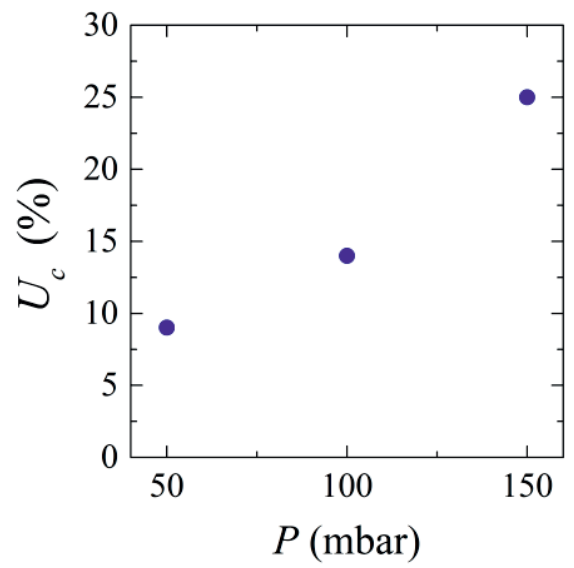

Figure 5. Percentage of unstable clogs $\left(U_{c}\right)$ for different system pressures $(P)$.

\section{Cake behavior}

In the microfluidic membrane system, microgels do not block the constriction for liquid flow [29], since microgel particles are still carried toward the channels after the system is clogged where they start to accumulate, forming a cake layer (Figure 6). We keep the microgel suspension flowing until a cake of approximately $250 \mu \mathrm{m}$ height is formed, and next investigate the extent of compression/deformation. The situation 


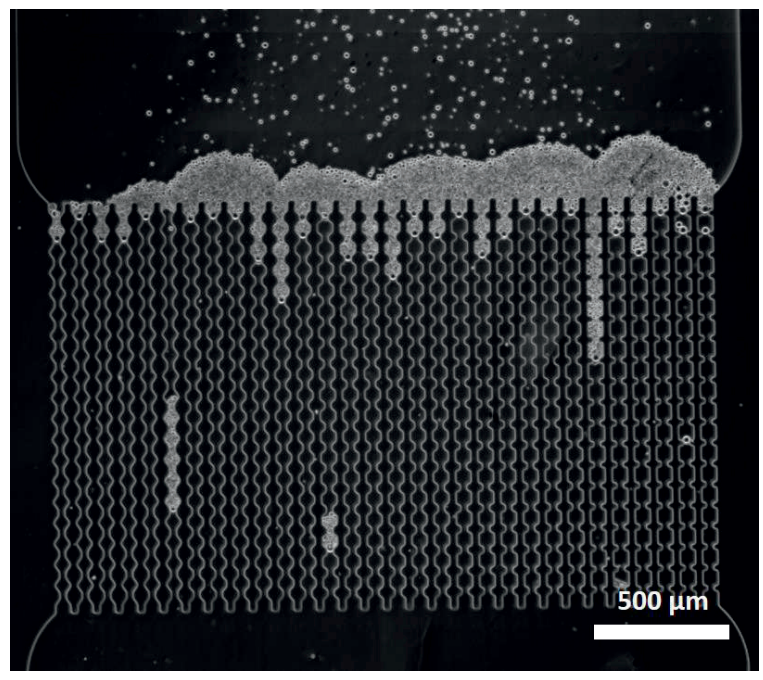

Figure 6. Cake layer forming on top of the channels as a result of channel clogging and subsequent particle deposition.

in Figure 6 is depicted after the device has been clogged and more particles have accumulated over a period of time. Depending on the efficiency of primary clogging, a pore may not carry any liquid (so no additional cake) or still be open for liquid transfer which leads to considerable layer formation and great differences in thickness over the width of the device.

Cake compression and relaxation experiments were done by gradually changing the applied pressure and analyzing the resulting images. The changes in pressure are gradual and do not provoke clog instability. The cake reaches steady state very rapidly, in a matter of seconds after a change of pressure, and recompression is almost totally reversible, since the area of the cake is almost the same as before relaxation (Appendix B). The actual difference in size may be influenced by some particles arriving at the cake layer, but since the swelling/deswelling processes are fast it can be expected that this only played a minor role if any.

To compare all relaxation/compression experiments we calculate the relative area by dividing the final cake area by the initial cake area. We re-plotted the relative area against the pressure applied for relaxation and compression (Figure 7). Please note that the data for each pressure shown in the graph correspond to an independent experiment. 


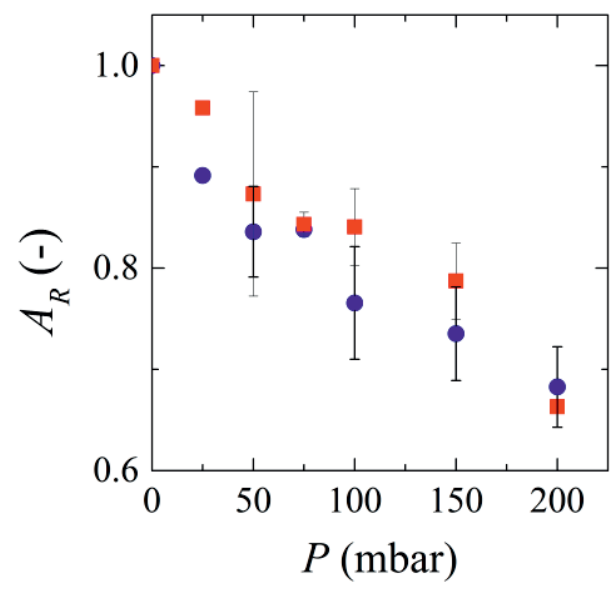

Figure 7. Relative cake area $\left(A_{R}\right)$ as a function of pressure $(P)$ for relaxation (red squares) and compression (blue circles). Data for each pressure correspond to independent experiments.

The relaxation curve seems a little lower than the compression curve (possibly as a result of particle-particle adhesion), but when we take the error bars into account, we can consider that both curves overlap. As mentioned, the system compresses and decompresses in the same degree; we are looking at the thermodynamic state. When considering the values in the graph, it is clear that the volume reduction of particles at these pressures is considerable ( $>30 \%$ at $200 \mathrm{mbar}$; the area in the graphs can be interpreted as a volume given the depth of the chip). This corresponds to a diameter change of $10 \%$ for 10 micrometer particles, which is sufficient to influence filtration considerably even at pressures that are used in practice for microfiltration.

Total reversibility of cakes formed by compressible particles such as wastewater sludge flocs has also been described [30], although some studies claim that total reversibility of compressible particles only means that the particles have not been compressed long / hard enough to form a cohesive cake layer [31,32]. We investigate this further using the microfluidic centrifugation methods.

\section{Centrifugation}

We use centrifugation experiments to simulate the compression and relaxation of the microgels when subjected to compressive forces only. The results shown in Figure 8 relate to the experiments done with the rectangular microchip chamber. The compression of the microgels at low $\mathrm{g}$-force seems similar to compression of cake 

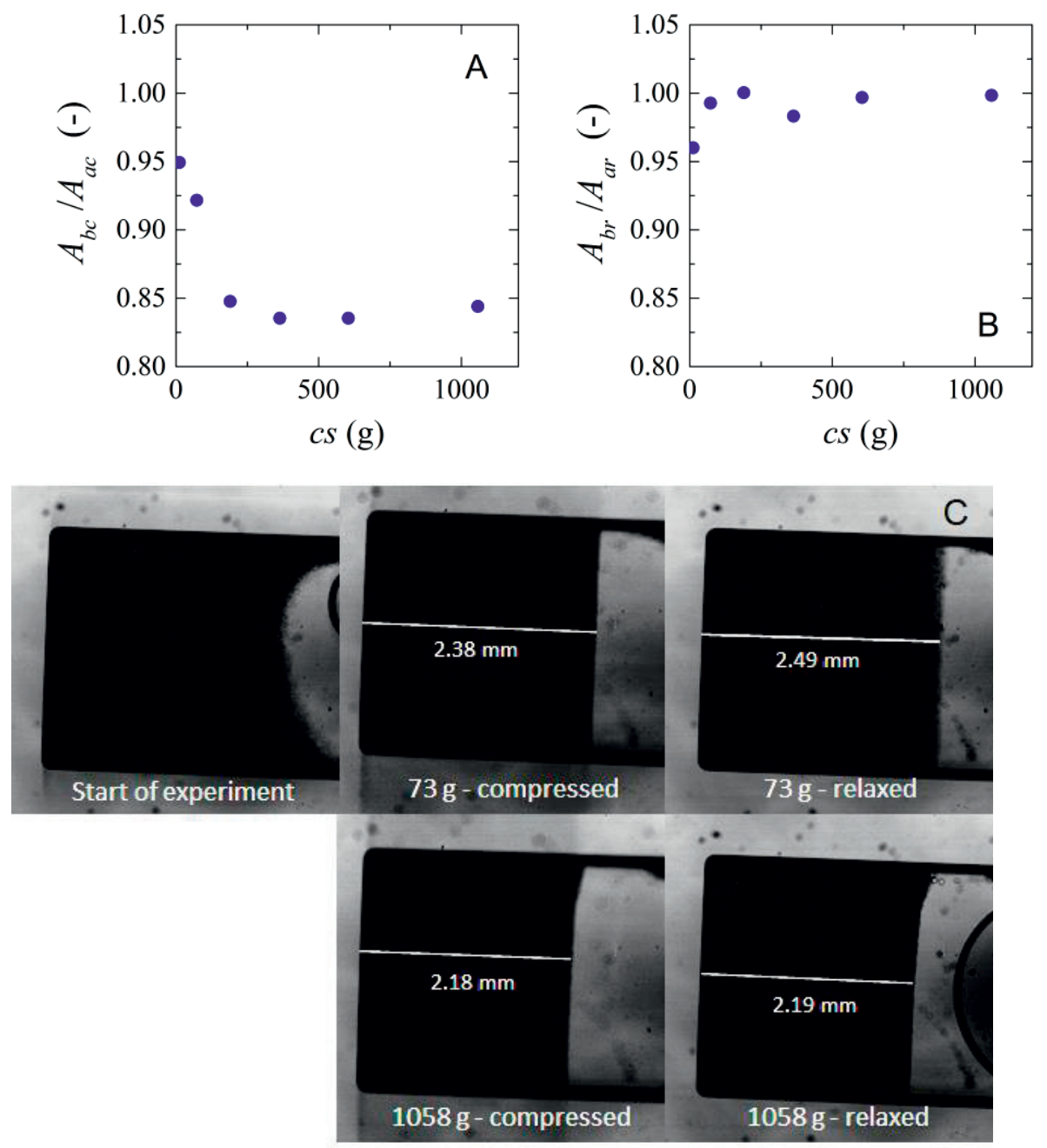

Figure 8. A) Compression data of microgels. Ratio between area after compression $\left(A_{a c}\right)$ and the area before compression $\left(A_{b c}\right)$ versus centrifugation speed $(c s)$. B) Relaxation data of microgels. Ratio between area after $\left(A_{a r}\right)$ and before relaxation $\left(A_{b r}\right)$ versus centrifugation speed ( $\left.c S\right)$. C) images showing the compressed and relaxed microgels at low and high number of $\mathrm{g}$-forces. Values of area were measured manually with an average measurement error $<0.5 \%$. 
layers in the microfluidic devices, in which a steep decrease in area is followed by a constant cake/deposit area (Appendix B).

During the relaxation experiments, we found that the extent to which the deposit relaxes depends on the applied g-force. A certain degree of relaxation can be observed in experiments in which the applied force was low (Figure 8B, and top images in c), but as the microgels are exposed to more and more force, the reversibility of the deposit is almost nonexistent within the experimental time frame (Figure 8C, bottom images). The maximum centrifugation speed where we can see some relaxation corresponds to a pressure of $36 \mathrm{mbar}$, whereas in the model microfiltration experiments, full relaxation was observed at pressures up to $200 \mathrm{mbar}$ (Figure 7). The plateau could be a combined result of compression behavior and changing the packing structure. However, in these experiments we did not have means to distinguish between both effects and have taken them as one. Since the same particles were used in both centrifugation and microfiltration experiments, the question is what causes the difference.

To answer this question, we carried out centrifugation experiments with the hourglass configuration chamber that has two areas connected by a small channel, as if it was a single pore, but of course with bigger dimensions. We tried to use the same microgels as for the previous experiments but the microgels quickly transfer to the bottom of the chamber as soon as the $\mathrm{g}$ force is increased, leading to the same behavior as observed in the square chamber. That is why we opted for the use of the larger Sephadex microgels, of which a part stays in the first section of the chamber (so, before the "pore") allowing us to observe "cake" behavior; please note that on the pictures of Figure 9, the right chamber contains the deposit and the left chamber is partly filled with water.

Figure $9 A \& B$ show that for the Sephadex particles in the hourglass configuration, total relaxation of the deposit occurs even at the highest force used (1058g), whereas this is not the case in the rectangular chamber. The difference between the square and hourglass configurations is related to the freedom that the compressed material has to reswell. This can be seen as the availability of solvent for capillary effects and also the fact that there is no layer of water above the deposit in the hourglass configuration to be "pushed" away. For this reason, the deposit can reswell. 

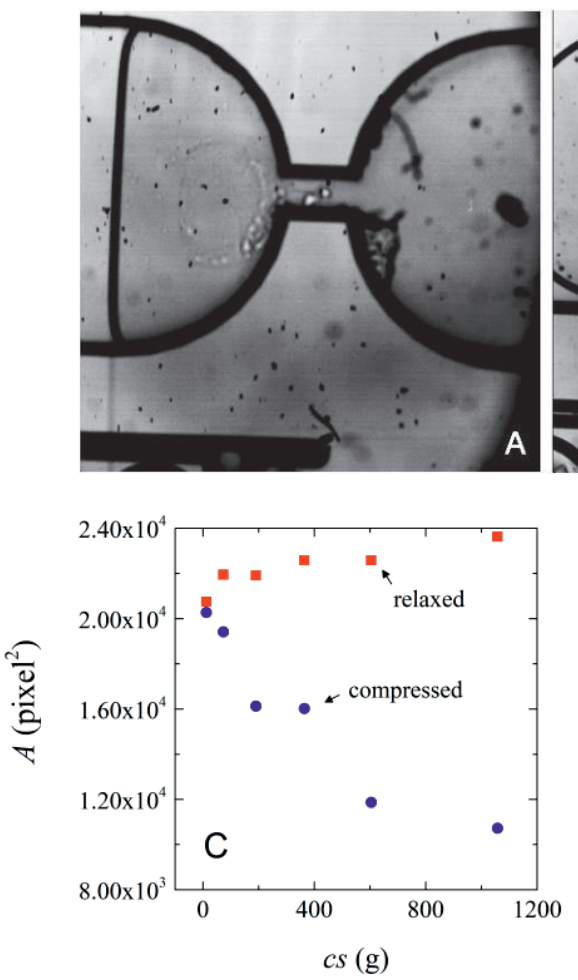
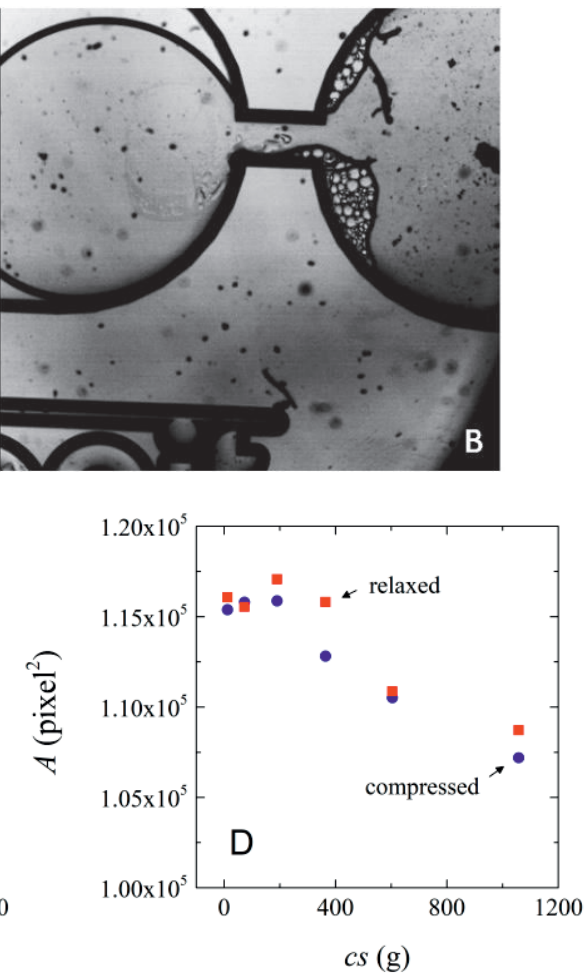

Figure 9. Area of Sephadex G100 microgels in the hourglass configuration microchips A) at $1058 \mathrm{~g}$. B) after 5 minutes of relaxation. Area of the microgels as function of the centrifugal speed at C) hourglass chamber configuration and D) squared chamber configuration. Please note that each centrifugal speed corresponds to an individual experiment. Blue circles represent compressed microgels and orange squares correspond to relaxed microgels; please note that in all cases Sephadex was used). Values of area were measured manually with an average measurement error $<0.5 \%$.

This also implies that full relaxation can be influenced by the design of the channel, (pore geometry). Furthermore, we expect these effects to be relevant for cake layers that build on top of membranes that, depending on the pore geometry can be removed more easily. As discussed before, for the microfluidic filtration system, even after the formation of the cake layer, there is still a flow of water between the microgels. This flow might avoid permanent/strong interactions between the particles as long as the applied pressure does not deform the particles too much. To be complete, differences in $\mathrm{g}$-forces due to the size of the cell are not expected to have played a role in our experiments. 


\section{Implications for membrane filtration}

When considering our findings in the light of membrane filtration, it is clear that large soft particles can clog pores effectively, but also can be pushed through the pores depending on many factors such as particle size, polydispersity of the particle suspension, applied pressure and pore configuration. Although currently not taken as a starting point for membrane process design, cake formation and behavior can be reversible depending on the applied pressure, and to some extent layer thickness. We expect that cake reversibility is an important parameter that co-determines removal of a cake layer by backwashing, and through that as well membrane life time.

With the use of centrifugation systems, we are able to extend our experiments to higher pressures, which could be of interest to for example ultrafiltration, nanofiltration, and reverse osmosis applications. It is also good to mention that the particle properties can be systematically varied, and the propensity of deswelling/deformation charted using the microfluidic systems presented here.

\subsection{Conclusions}

In this chapter, we investigated the behavior of soft microgels in microfluidic filtration systems as well as in centrifugation systems in order to emulate various situations occurring during membrane filtration. The propensity to pore clogging, and the position (depth) at which this takes place in a model membrane, depends on the applied pressure. At low pressure pores block immediately, while at higher pressures microgels deform and can be pushed through pores that are smaller than the microgel diameter, to potentially block pores deeper in the model membrane. At even higher pressure, particles can be more and more pushed through the pores, even after initially blocking them.

When forming a cake layer on top of the model membrane, we found that cake compression may be as high as $30 \%$ but it is totally reversible. These results are in line with those obtained with the so-called hourglass configuration in which a thin layer of microgels was present, but are in complete contrast with results obtained with a rectangular configuration containing a thick layer of microgels that compressed irreversibly. This shows that the presence of water near the microgels is of essence for reversibility of cake/deposits.

Our results are useful for the understanding of membrane filtration of compressible particles and shed a new light on cake layers and their ease of removal. We clearly showed that soft particles can be pushed through pores that are smaller than their original dimension, and deformation/deswelling should be taken into consideration 
for membrane design and process optimization. The extent to which this takes place can be quantified using the model systems that we presented here.

\section{Acknowledgements}

We thank Ties van de Laar for writing the MATLAB code for image treatment. 


\section{Appendix B. Supplementary data}
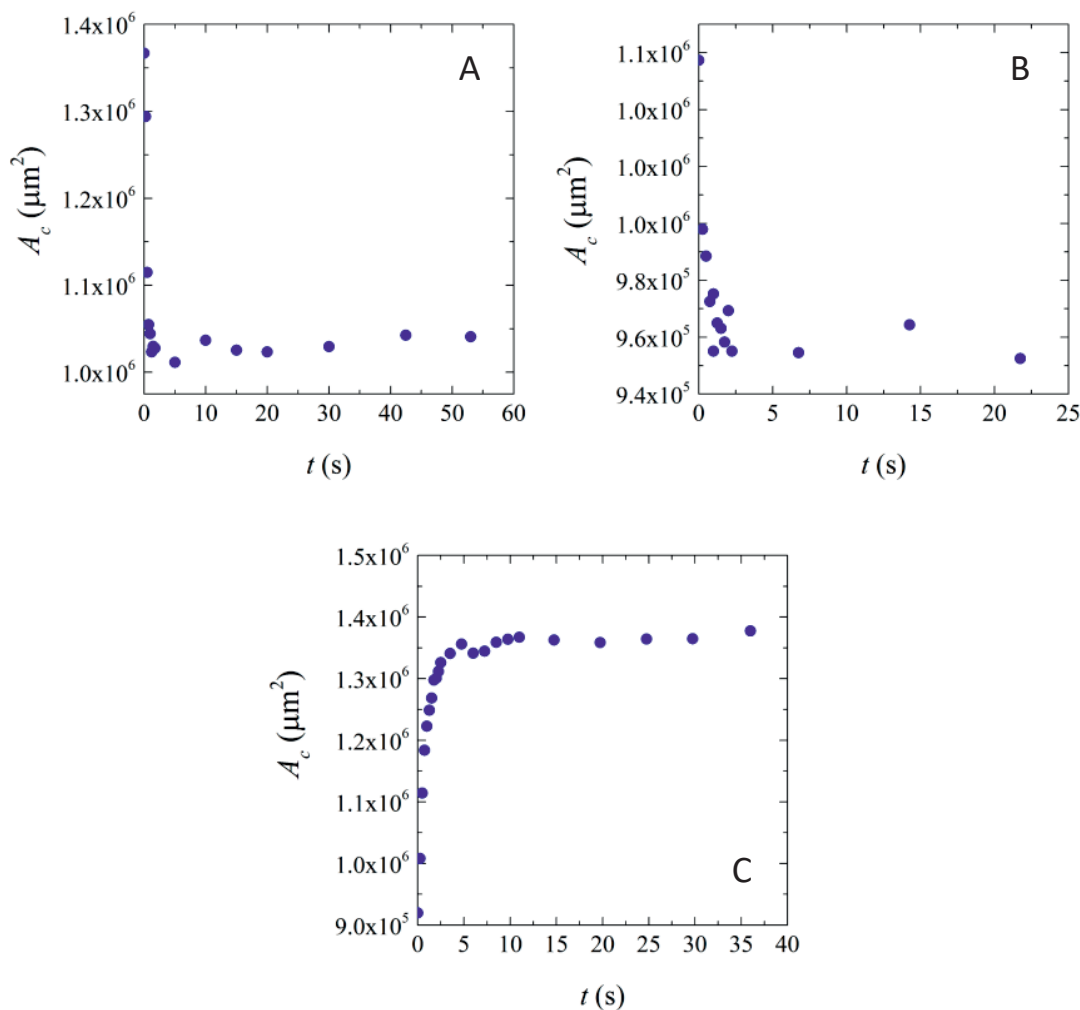

Figure 10. Cake area $\left(A_{c}\right)$ as a function of time $(t)$ A) from 0 to $\left.50 \mathrm{mbar}, \mathrm{B}\right)$ from 50 to 100 mbar and C) from 100 to 0 mbar. 


\section{References}

[1] T. Sohail, B. Nadler, On the indentation of a fluid-filled spherical particle, Int. J. Mech. Sci. 75 (2013) 305-315.

[2] Y.L. Lin, D. M. Wang, W.M. Lu, Y.S. Lin, K.L. Tung, Compression and deformation of soft spherical particles, Chem. Eng. Sci. 63 (2008) 195-203.

[3] S.F. Hansen, B. Petrat-Melin, J.T. Rasmussen, L.B. Larsen, M.S. Ostenfeld, L. Wiking, Placing pasteurisation before or after microfiltration impacts the protein composition of milk fat globule membrane material, Int. Dairy J. 81 (2018) 35-41.

[4] O. Abdelhedi, R. Nasri, L. Mora, M. Jridi, F. Toldrá, M. Nasri, In silico analysis and molecular docking study of angiotensin I-converting enzyme inhibitory peptides from smooth-hound viscera protein hydrolysates fractionated by ultrafiltration, Food Chem. 239 (2018) 453-463.

[5] O.A. Kovaleva, S.I. Lazarev, and S.V. Kovalev, Effect of transmembrane pressure on microfiltration concentration of yeast biomass, Pet. Chem. 57 (2017) 974-982.

[6] Y. Jeong, Y. Kim, Y. Jin, S. Hong, C. Park, Comparison of filtration and treatment performance between polymeric and ceramic membranes in anaerobic membrane bioreactor treatment of domestic wastewater, Sep. Purif. Technol. 199 (2018) 182-188.

[7] L. Wang, W. Liang, W. Chen, W. Zhang, J. Mo, K. Liang, B. Tang, Y. Zheng, F. Jiang, Integrated aerobic granular sludge and membrane process for enabling municipal wastewater treatment and reuse water production, Chem. Eng. J. 337 (2018) 300-311.

[8] A.M.C. Van Dinther, C.G.P.H. Schroën, R.M. Boom, Separation process for very concentrated emulsions and suspensions in the food industry, Innov. Food Sci. Emerg. Technol. 18 (2013) 177-182.

[9] J. Woods, J. Pellegrino, J. Burch, Generalized guidance for considering poresize distribution in membrane distillation, J. Membr. Sci. 368 (2011) 124-133.

[10] C.W. Hsu, Y.L. Chen, Migration and fractionation of deformable particles in microchannel, J. Chem. Phys. 133 (2010) 034906.

[11] G. Cevc, D. Gebauer, Hydration-driven transport of deformable lipid vesicles through fine pores and the skin barrier, Biophys. J. 84 (2003) 1010-1024.

[12] T.F. Headen, S.M. Clarke, A. Perdigon, G.H. Meeten, J.D. Sherwood, M. Aston, Filtration of deformable emulsion droplets, J. Colloid Interface Sci. 304 (2006) 562-565.

[13] L.E. Head, M.R. Bird, Backwashing of tubular ceramic microfilters fouled with milk protein isolate feeds, J. Food Process Eng. 36 (2013) 228-240. 
[14] A. Ostadfar, A.H. Rawicz, M. Gitimoghaddam, Application of backwashing to increase permeate flux in bioparticle separation, J. Med. Biol. Eng. 33 (2013) 478-485.

[15] J. de Jong, R.G.H. Lammertink, M. Wessling, Membranes and microfluidics: a review, Lab Chip 6 (2006) 1125-1139.

[16] Y.H. See Toh, X.X. Loh, K. Li, A. Bismarck, A.G. Livingston, In search of a standard method for the characterisation of organic solvent nanofiltration membranes, J. Membr. Sci. 291 (2007) 120-125.

[17] K.J. Hwang, P.Y. Su, E. Iritani, N. Katagiri, Compression and filtration characteristics of yeast-immobilized beads prepared using different calcium concentrations, Sep. Sci. Technol. 51 (2016) 1947-1953.

[18] Y. Xin, M.W. Bligh, A.S. Kinsela, T.D. Waite, Effect of iron on membrane fouling by alginate in the absence and presence of calcium, J. Membr. Sci. 497 (2016) 289-299.

[19] W. Li, X. Liu, Y.-N. Wang, T.H. Chong, C.Y. Tang, A.G. Fane, Analyzing the evolution of membrane fouling via a novel method based on 3D optical coherence tomography imaging, Environ. Sci. Technol. 50 (2016) 6930-6939.

[20] Y. Gao, S. Haavisto, W. Li, C.Y. Tang, J. Salmela, A.G. Fane, Novel approach to characterizing the growth of a fouling layer during membrane filtration via optical coherence tomography, Environ. Sci. Technol. 48 (2014) 14273-14281.

[21] P. Bacchin, A. Marty, P. Duru, M. Meireles, P. Aimar, Colloidal surface interactions and membrane fouling: investigations at pore scale, Adv. Colloid Interface Sci. 164 (2011) 2-11.

[22] H. Di, G. J.O. Martin, D.E. Dunstan, A microfluidic system for studying particle deposition during ultrafiltration, J. Membr. Sci. 532 (2017) 68-75.

[23] E. Dressaire, A. Sauret, Clogging of microfluidic systems, Soft Matter 13 (2017) $37-48$.

[24] R. van Zwieten, T. van de Laar, J. Sprakel, K. Schroën, From cooperative to uncorrelated clogging in cross-flow microfluidic membranes, Sci. Rep. 8 (2018) 5687.

[25] I. Bouhid de Aguiar, T. van de Laar, M. Meireles, A. Bouchoux, J. Sprakel, K. Schroën, Deswelling and deformation of microgels in concentrated packings, Sci. Rep. 7 (2017) 10223.

[26] T. van de Laar, S. ten Klooster, K. Schroën, J. Sprakel, Transition-state theory predicts clogging at the microscale, Sci. Rep. 6 (2016) 28450.

[27] T. Krebs, D. Ershov, C.G.P.H. Schroën, R.M. Boom, Coalescence and compression in centrifuged emulsions studied with in situ optical microscopy, Soft Matter 9 (2013) 4026. 
[28] "Rasband, W.S., ImageJ, U. S. National Institutes of Health, Bethesda, Maryland, USA, http://imagej.nih.gov/ij/, 1997-2016."

[29] Y. Li, O.S. Sarlyer, A. Ramachandran, S. Panyukov, M. Rubinstein, E. Kumacheva, Universal behavior of hydrogels confined to narrow capillaries, Sci. Rep. 5 (2015) 1-11.

[30] B. Teychene, C. Guigui, C. Cabassud, Engineering of an MBR supernatant fouling layer by fine particles addition: a possible way to control cake compressibility, Water Res. 45 (2011) 2060-2072.

[31] M.K. Jørgensen, T.V. Bugge, M.L. Christensen, K. Keiding, Modeling approach to determine cake buildup and compression in a high-shear membrane bioreactor, J. Membr. Sci. 409-410 (2012) 335-345.

[32] D. Jeison, J.B. van Lier, Cake formation and consolidation: main factors governing the applicable flux in anaerobic submerged membrane bioreactors (AnSMBR) treating acidified wastewaters, Sep. Purif. Technol. 56 (2007) 71-78. 


\section{Conformational changes influence clogging behavior of micrometer-sized microgels in idealized multiple constrictions}




\section{Abstract}

Clogging of porous media by soft particles has become a subject of extensive research in the last years and the understanding of the clogging mechanisms is of great importance for process optimization. The rise in the utilization of microfluidic devices brought the possibility to simulate membrane filtration and perform in situ observations of the pore clogging mechanisms with the aid of high-speed cameras.

In this work, we use microfluidic devices composed by an array of parallel channels to observe the clogging behavior of micrometer sized microgels. It is important to note that the microgels are larger than the pores/constrictions. We quantify the clog propensity in relation to the clogging position and particle size and find that the majority of the microgels clog at the first constriction independently of particle size and constriction entrance angle. We also quantify the variations in shape and volume (2D projection) of the microgels in relation to particle size and constriction entrance angle. We find that the degree of deformation increases with particle size and is dependent of constriction entrance angle, whereas, changes in volume do not depend on entrance angle. 


\subsection{Introduction}

The observation of soft particles going through constrictions has become a subject of extensive research in the last years [1-3]. Depending on the properties and size of the particle relative to the pore size, various mechanisms occur. Micrometer sized soft particles reduce their size or deform to go through constrictions that are smaller than their diameter, whereas hard particles would not be able to do so. Small soft particles moving through larger pores would not need to deswell, or deform, leading to colloidal interactions playing a more prominent role $[4,5]$.

For hard particles, clogging can happen through sieving effects, bridging or agglomeration, depending on the size (distribution) of the particles, and their interactions. Since these particles are not able to modify their conformation, the clogging propensity is determined by process conditions, and the ratio of channel to particle (agglomerate) dimension [6]. When using a suspension of colloidal soft particles, clogging can happen through agglomeration or the formation of arches, as was the case for hard particles; but the pore size is no longer the strict gate keeper for particle permeation [7,8]. Soft particles larger than constrictions may be pushed all the way through [1]; whereas the largest particles have highest propensity to get stuck in the constrictions and clog the channels [9].

Depending on particle size and constriction geometry, soft particles can use two mechanisms to accommodate the pressure drop generated by the clog they generate: deformation and deswelling [10,11]. A soft particle deforms when it assumes a different shape to adjust to the surrounding environment. Deformation can also be observed in emulsion droplets [2,12]; however, unlike emulsion droplets, soft particles such as microgels are also able to accommodate some of the external pressure by deswelling. Deswelling happens when particles expel solvent, therewith effectively decreasing their volume. It has been already reported that both deformation and deswelling of constricted soft particles occur simultaneously (Chapter 3). The challenge that we face is to understand how these modifications occur, and use that knowledge in the design of processes where these effects occur (such as membrane filtration).

Microfiltration is a membrane process based on size exclusion that has pressure as a driving force. The size exclusion concept says that what is larger than the size of the pore will be retained while what is smaller will go through $[13,14]$. In a process where soft particles are present, the concept of size exclusion should be used with care since, as soft particles are able to modify their conformation under pressure making the size 
exclusion concept not very straightforward, unless when linked to the effective size of the soft particles under process conditions.

Many direct and indirect methods have been reported in the literature to observe and describe the filtration behavior of soft particles. Some indirect methods include the quantification of soft particles in the permeate, and modeling of process parameters $[12,15]$. Direct observation methods have also been described and mostly include the use of microfluidic devices coupled with optical microscopes and high-speed cameras [16-18]. Microfluidic devices are flexible in their design and allow for endless variation in conformation. In our work, the microfluidic devices generally contain constricted channels and soft particles flow through them. We chose to work with microgels as model particles due to their ease of fabrication and tunable properties.

In this work, we use microfluidic devices to observe the clogging behavior of microgels larger than the constrictions. We quantify the clog propensity in relation to the clogging position and particle size and find that the majority of the microgels clog at the first constriction independent of particle size and constriction entrance angle. We also quantify the variations in shape and volume (2D projection) of the microgels in relation to particle size and constriction entrance angle. We find that the degree of deformation increases with particle size and is dependent on constriction entrance angle, whereas, changes in volume are not dependent on entrance angle.

\subsection{Material and methods}

\section{Microgel synthesis and characterization}

Polyacrylamide (pAAm) microgels are used as model particles for the channel clogging experiments. The microgels were produced via the emulsion templating technique as described in previous work [16]. For the preparation of the microgels, we start by mixing $100 \mathrm{~mL}$ kerosene with $1 \%$ of the surfactant polyglycerol polyricinoleate (PGPR90). In a separate container we prepare the monomer solution with $10 \mathrm{ml}$ of water, $0.1 \mathrm{M}$ sodium hydroxide solution to set the $\mathrm{pH}$ at 8.5, $2.5 \mathrm{~g}$ of acrylamide, $50 \mathrm{mg}$ of potassium persulfate (KPS) and $25 \mathrm{mg}$ of N,N'methylenebisacrylamide (BIS) as the crosslinker at $1 \%$ wt as compared to the total monomer content. The monomer solution is added to the content of the round bottom flask and the aqueous phase is emulsified into the oil phase under high shear with a rotor-stator mixer for three minutes. We then close the round bottom flask with a rubber septum and bubble the emulsion with nitrogen for 20 minutes to remove oxygen. We subsequently place the round bottom flask on a stirring plate on ice and we inject $1 \mathrm{ml} \mathrm{N}, \mathrm{N}, \mathrm{N}^{\prime}, \mathrm{N}^{\prime}$-tetramethylethylenediamine (TEMED) to trigger the polymerization. The reaction time is $2-3$ hours. After, we precipitate the 
microgels in cold methanol and the microgels are cleaned by repeated centrifugation and resuspension steps, first in methanol to remove excess kerosene and surfactant, and finally in water. The microgel suspension is stored at $4{ }^{\circ} \mathrm{C}$. The microgels are micrometer sized and have a polydisperse size distribution. The diameter of the microgels range from $3 \mu \mathrm{m}$ to $50 \mu \mathrm{m}$ with a Sauter mean diameter (D[3,2]) of $10 \mu \mathrm{m}$. Size distributions were measured by laser diffraction (Malvern Mastersizer 3000). The microgels were used suspended in water at $\sim 0.1 \%$ vol.

\section{Microfluidics}

The microfluidic devices we use to simulate membrane filtration are composed of a main channel with an inlet and an outlet. An array of smaller parallel channels is connected to the main channels to simulate the pores of a membrane. The channels have 5 different entrance angles ( 6 channels for each angle) varying from $0^{\circ}$ to $55^{\circ}$ (Figure 1 and Figure 2) [19]. The microfluidic devices were produced by soft lithography and coupled to an optical microscope equipped with a high-speed camera to allow in situ observation of the filtration process. The devices were connected via Teflon tubing to a pressure controller (Elveflow OB1-MK3). The filtration experiments were conducted at $100 \mathrm{mbar}$ and observed at relatively low magnification (2.5x) until all the channels were clogged. We consider the system completely clogged when there is at least one particle permanently stuck in every channel in the time frame observed (average of 10 minutes). After system clogging was complete, we increased the magnification to $20 \mathrm{x}$, zooming in on a single particle stuck in a pore and gradually increased the pressure up to $800 \mathrm{mbar}$ to force the particle to go through the constriction. With this experiment we expect to be able to observe particle's change in conformation and/or volume when going through a constriction.

\section{Image analysis}

Self-written MATLAB scripts are used to analyze the images; for the clogging experiments, we determine the average clog constriction position, so how deep each channel clogs. For experiments focusing on the single particle behavior, we select images from the sequence obtained with a high-speed camera: typically showing the particles in the middle of the constriction, on the verge of being pushed all the way through. We use these images to determine the degree of deformation of the particles as previously described [16]. Briefly, we rewrote the sphericity equation in terms of the area and the perimeter of the microgel in a $2 \mathrm{D}$ image which leads to the following equation to calculate this sphericity parameter $\Psi$ : 


$$
\Psi=\frac{2 \sqrt{\pi N_{a}}}{N_{\operatorname{circ}}}
$$

where $N_{a}$ is the number of pixels in the area of a microgel and $N_{\text {circ }}$ is the number of pixels in the circumference of the microgel area.

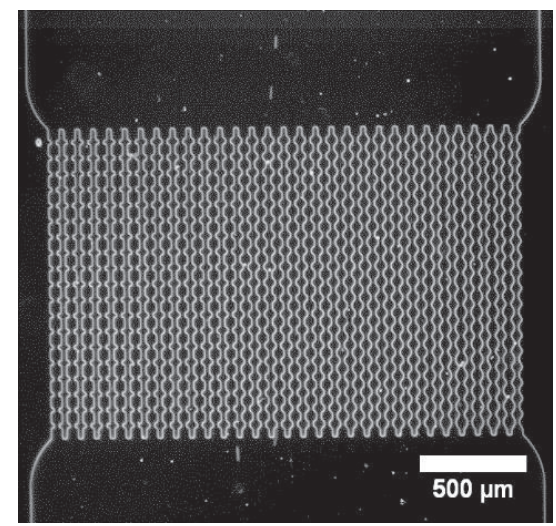

Figure 1. Optical microscopy image of the array of channels that compose the microfluid device at $2.5 \mathrm{x}$ magnification. There are 30 parallel channels with five different entrance angles. From left to right: $0,20,35,45,55^{\circ}$. Scale bar denotes 500 $\mu \mathrm{m}$.
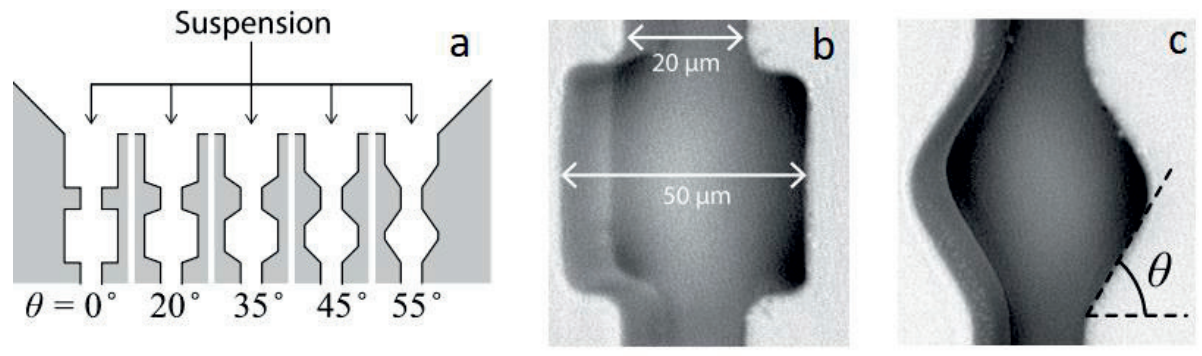

Figure 2. Schematic representation of the channels and their different entrance angles, b) internal dimensions of the constrictions, depth of the channels is $40 \mu \mathrm{m}, \mathrm{c}$ ) constriction representing the location where the angles are measured. Reproduced with permission from Nature (van de Laar et al. [19]). 


\subsection{Results and discussion Average clog position in the channels}

In this study, we observe the behavior of individual particles at local scale through optical microscopy. During the clogging experiments, the channels of the microfluidic devices clog at different positions. To analyze whether this position is related to the particle size or to the applied pressure, we plot the clog propensity for each clog position for three different filtration pressures (Figure 3A-C). Clog propensity is the percentage of clogs in a determined position in relation to the total amount of observed clogs in all channels. For a better understanding of the data, we classified the particles according to their size in three different size ranges within the same experiment. From our observations it is clear that most of the channels clog at the first constriction, independently of particle size or applied pressure. There is a slight trend for smaller particles to clog at higher constriction number (deeper in the channel), which seems obvious since smaller particles are more likely to be pushed through a constriction, having to go through less shape modification (deswelling and deformation).

At 50 mbar applied pressure, we can see that clogging happens only in the first position for particles larger than $30 \mu \mathrm{m}$. For smaller particles, this happens within the first eight constrictions. As we increase the applied pressure to $100 \mathrm{mbar}$, the particles may be forced to go deeper into the channels before clogging it. Particles larger than $30 \mu \mathrm{m}$ have a very high propensity to clog at the first constrictions and smaller particles have higher propensity to $\operatorname{clog}$ at deeper positions. As we increase the applied pressure to $150 \mathrm{mbar}$, we see that particles smaller than $25 \mu \mathrm{m}$ do not clog the channels anymore (Figure 3C,D). The pressure is high enough to promote the modification of their conformation and for that reason they can be pushed all the way through the channels. Slightly larger particles show a high propensity to clog at the beginning of the channels, as part of the natural sieving effect. Given the process conditions, these particles cannot modify their shape sufficiently to move through the constrictions, and thus clog the channels. The size distribution of the particles that clog the channels at different pressures can be seen in Figure 3D. We can see that the system selectivity changes with the variation in pressure due to the changes in conformation of the microgels.

The channels of the microfluidic devices used in this work have a variety of entrance angles. In Chapter 3, we found that the entrance angles do not have an influence on overall clogging behavior of micrometer-sized particles that are smaller than the pore. Here we investigate larger particles in detail. 

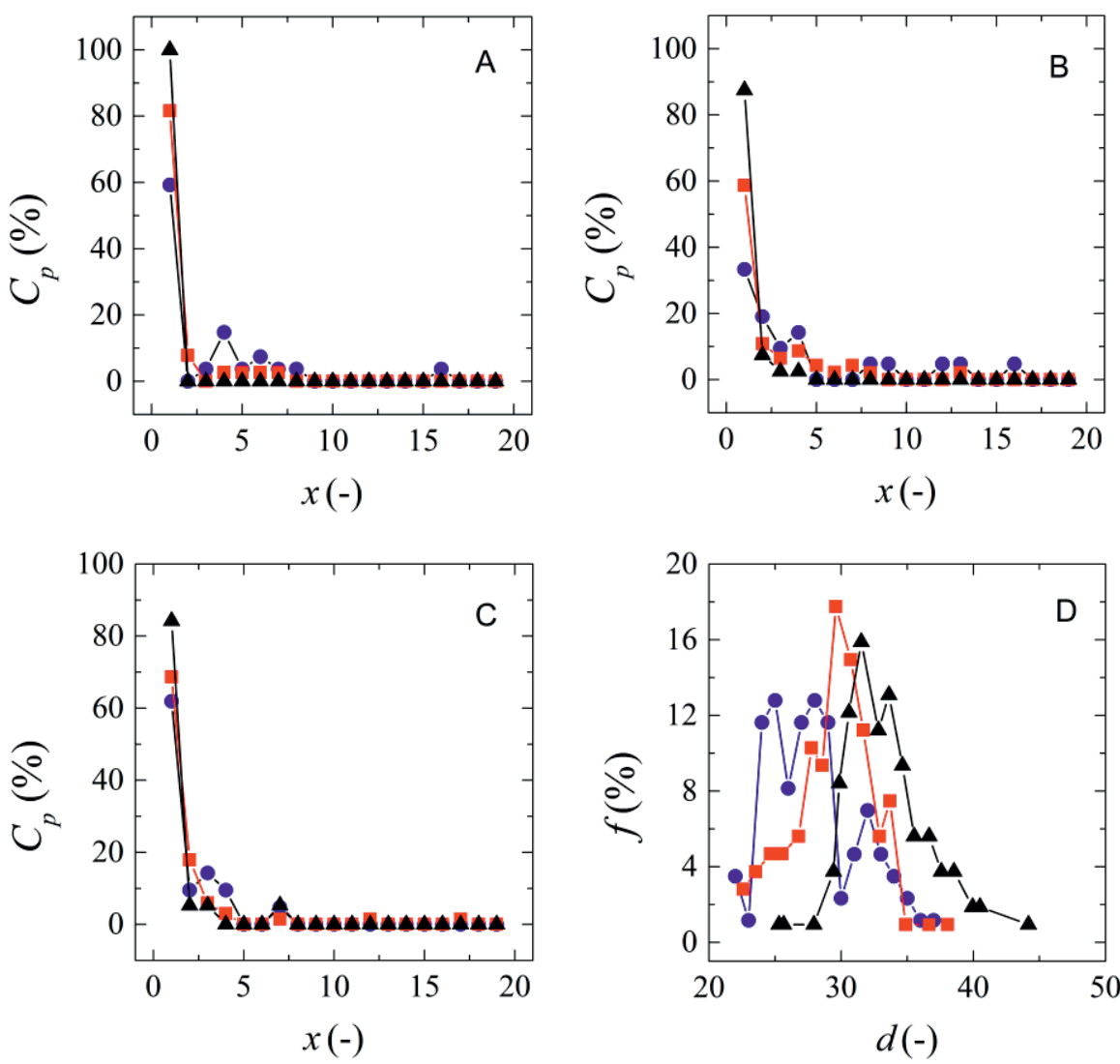

Figure 3. Relationship between clog propensity and clog position (how deep in the channel the clog stabilizes). At A) 50 mbar, B) 100 mbar and C) 150 mbar. Blue symbols represent particles between 25 and $30 \mu \mathrm{m}$. Red symbols represent particles between 31 and $35 \mu \mathrm{m}$ and black symbols represent particles between 36 and $40 \mu \mathrm{m}$. D) Size distribution of the microgels that clog the pores at $50 \mathrm{mbar}$ (blue symbols), $100 \mathrm{mbar}$ (red symbols) and $150 \mathrm{mbar}$ (black symbols). Lines are to guide the eye.

\section{In situ observation of clogging microgels}

Whenever soft particles are forced through pores that are smaller than their diameter, the particles change their shape. Figure 4 shows microgels before going through a constriction and during their passage through the constriction. It is easy to see that the microgels go through changes in shape (deformation); however, it is harder to quantify deformation and to analyze whether microgels also deswell (lose volume). For that reason, we use image analysis to determine the degree of deformation and deswelling of the particles. 

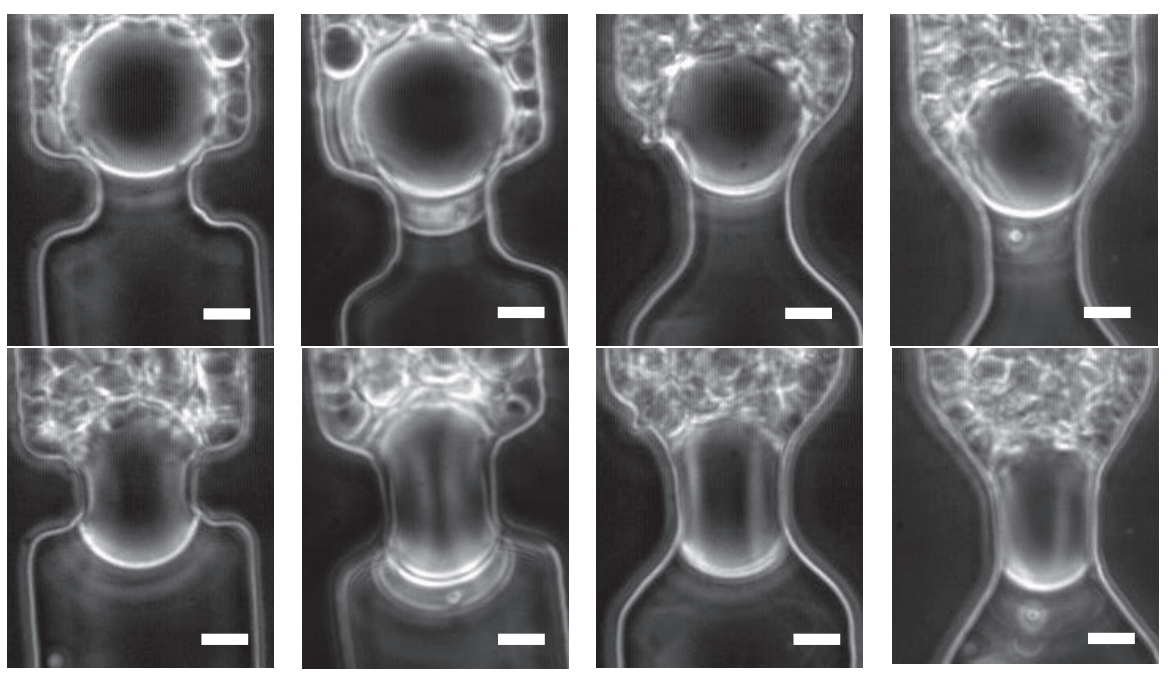

Figure 4. Optical microscopy (20x) images of microgels before constrictions (top) and in the middle of constrictions (bottom), for four different entrance angles going from left to right: $0,20,45$ and $55^{\circ}$. Scale bars denote $10 \mu \mathrm{m}$.

To determine the degree of deformation of the microgels before and during their passage though the pores (middle of the constriction), we determine the sphericity parameter of the microgels from the images (Equation (1).

For comparison, the sphericity of a perfect sphere is 1.00 , the sphericity of an ellipse is 0.93 , and the sphericity of a dumbbell is approximately 0.80 . These are also the shapes we will use throughout the chapter to describe the deformed microgels.

Figure $5 \mathrm{~A}$ shows the plot of the sphericity of microgels at different entrance angles for microgels with different sizes. The sphericity of microgels with diameters varying from 24 to $26 \mu \mathrm{m}$ going through constrictions does not depend on the entrance angle of the channels and their values are very close to 1 , which implies that these microgels do not deform much to go through the constrictions. This is not surprising since the constrictions have a size of $20 \mu \mathrm{m}$. As the microgels increase in size (diameters from 27 to $30 \mu \mathrm{m}$ ), they tend to have lower sphericity values (Figure 5B and Figure 5D), which is more pronounced at low channel entrance angles (Figure 5A). This means that the microgels are deformed as a dumbbell when going through low entrance angle constrictions (Figure 5C). On the other hand, at high entrance angles the microgels tend to assume a more elliptical shape, as reflected in their sphericity values. 

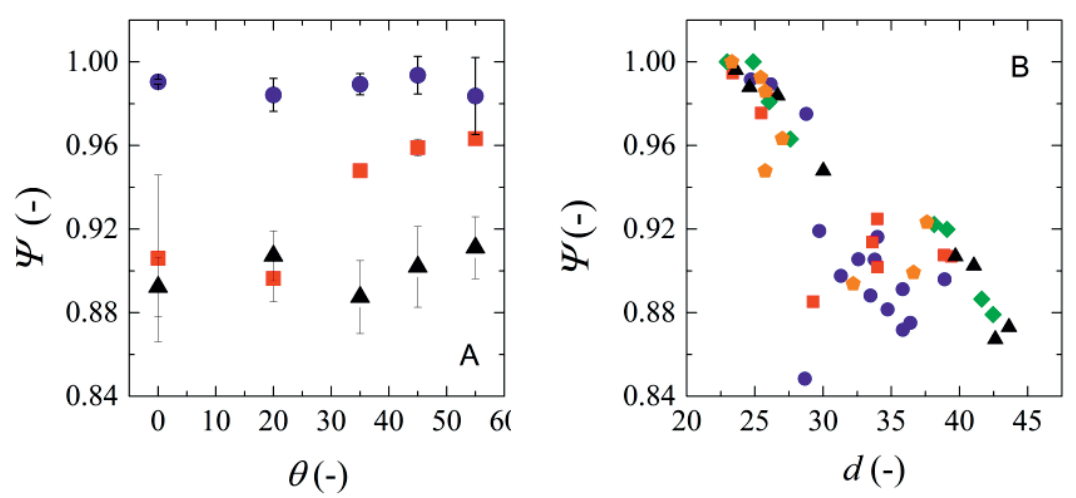

C
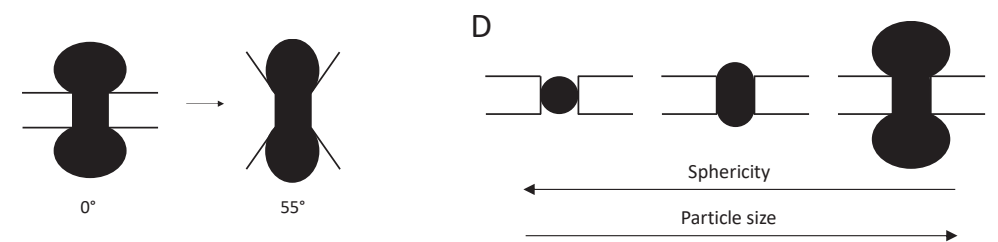

Figure 5. A) Microgels sphericity $(\Psi)$ variation with entrance angle $(\theta)$. Blue circles represent data obtained for microgels with diameters between 24 and $26 \mu \mathrm{m}$, red squares for diameters between 27 and $30 \mu \mathrm{m}$, and black triangles for diameters higher than $30 \mu \mathrm{m}$. B) Sphericity as function of particle diameter. Blue circles represent entrance angles of $0^{\circ}$, red squares $20^{\circ}$, black triangles $35^{\circ}$, green diamonds $45^{\circ}$ and orange pentagons $55^{\circ}$. C) Scheme representing the difference in shape that microgels assume at different entrance angles. D) Scheme representing the change in shape of at increasing microgel size.

For microgels with diameters larger than $30 \mu \mathrm{m}$, we observe an independence of microgel sphericity on entrance angle, and also find low sphericity values (Figure 5A). This means that these microgels are so large that they have to deform to a dumbbell shape independently of the shape of the constriction. We also have illustrated a number of effects in Figure 6, in which we show images of microgels of similar size going through low and high entrance angle constrictions (top part), and it is clear that both microgels deform into similar dumbbell shapes. When using different sizes of microgels going through the same constriction as illustrated in the bottom images of Figure 6, it is also clear that they assume different shapes. 

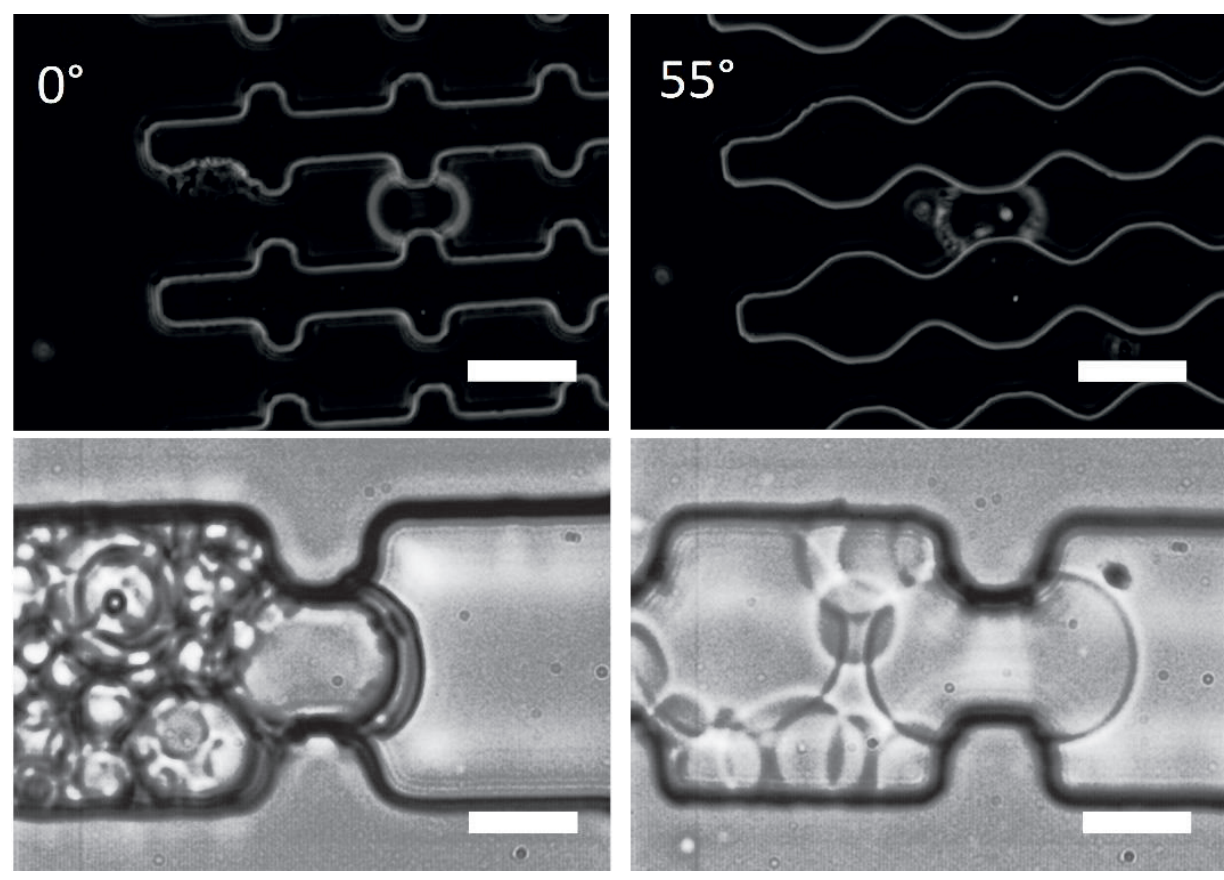

Figure 6. Top: Optical microscopy images (10x) showing microgels of similar size in the middle of constrictions of different entrance angles. Particles have diameters of 43 and $41 \mu \mathrm{m}$, respectively. Scale bars denote $50 \mu \mathrm{m}$. Bottom: Optical microscopy images (20x) of microgels with different sizes going through constrictions with the same entrance angle $\left(0^{\circ}\right)$. Particles have diameters of 27 and $43 \mu \mathrm{m}$, respectively. Scale bars denote $20 \mu \mathrm{m}$.

\section{Particle compression versus deformation}

To quantify compression/deswelling of the particle we use the ratio between the 2D projection area of the particle image in the middle of the constriction $\left(A_{2}\right)$ and the area of the particle before the constriction $\left(A_{1}\right)$. We plot the change in area as function of the sphericity (Figure 7) for particles of similar size ranges, this time for smaller size intervals as the ones used before (in Figure 3). Small particles may lose a bit of area, but the sphericity is still very close to 1 since the particles hardly need to deform or deswell to pass the constriction. As the size of the microgels increases, both sphericity values and change in area decrease since larger microgels have to modify themselves more to go through the pores. As for the degree of each modification (deformation or deswelling) we see that deswelling plays an important role as does deformation. Larger particles obviously need to deform more to pass through the constriction, and possibly deswell to some extent. Both processes are time dependent, and since we binned all data irrespective of the applied pressure or 


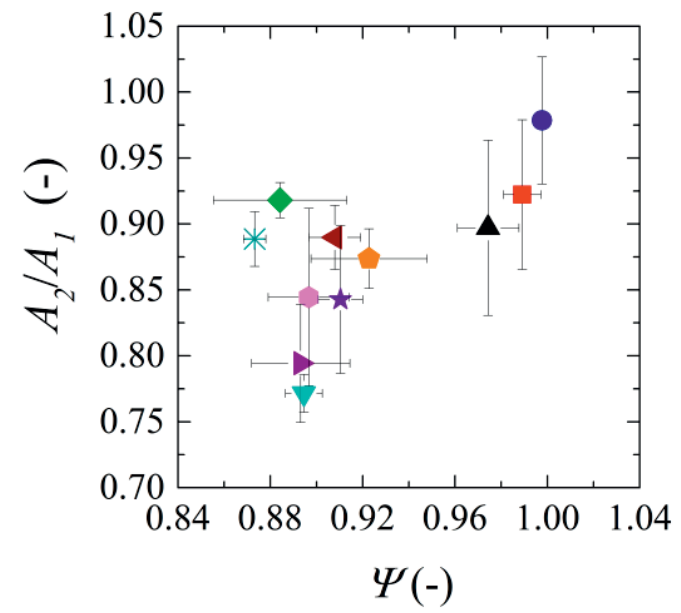

Figure 7. Comparison between deswelling $\left(A_{2} / A_{1}\right)$ and deformation $(\Psi)$ for microgels with diameters varying from 22 to $43 \mu \mathrm{m}$. Points represent binned data: $22-23 \mu \mathrm{m}$ (blue circle), 24-25 $\mu \mathrm{m}$ (red square), 26-27 $\mu \mathrm{m}$ (black triangle), 28-29 $\mu \mathrm{m}$ (green diamond), 30-31 $\mu \mathrm{m}$ (orange pentagon), 32-33 $\mu \mathrm{m}$ (brown left arrow), 34-35 $\mu \mathrm{m}$ (purple right arrow), 36-37 $\mu \mathrm{m}$ (pink hexagon), 37-39 $\mu \mathrm{m}$ (lilac star), 40-41 $\mu \mathrm{m}$ (teal inverted triangle), $42-43 \mu \mathrm{m}$ (turquoise asterisk).

entrance angle, it could be that some differences are due to the kinetics of these processes.

Also, large microgels $(>40 \mu \mathrm{m})$ assume a dumbbell shape while going through the pores (Figure 7). The dumbbell shape allows for a more localized compression, i.e. the deswelling occurs only at the part that is going through the constriction and as soon as that part is released, it swells back while another part is being compressed. In this way the microgels do not deswell as a whole but part by part and reswell quickly as soon as they come out of the constriction. For this reason, the variation in area for larger microgels as seen in Figure 6 is again comparable to the ones found in smaller microgels. As mentioned previously, the angle and the applied pressure (kinetics) may have played a role here, but for now we consider this outside the scope of our analysis, and we limit ourselves to the overall behavior.

\subsection{Discussion}

In previous work [16], we investigated the behavior of microgel packings at different osmotic pressures, and found that microgels use both deswelling and deformation to accommodate pressure to different extents. The composition of the microgels changes with increasing pressure since they lose volume, becoming more 
concentrated which also influences their deswelling, and deformation behavior. In our current observations on microgels under dynamic conditions, we see that this also holds. The microgels both deform and lose volume to accommodate applied forces as illustrated in Figure 7.

We observe that microgels clog individually due to the fact that they are soft and large. For this reason, they are not likely to clog by forming arches or agglomerate (no colloidal effects) [2,6]. However, as they are soft, they can also go through constrictions that are smaller than their diameter by changes in shape and volume [10].

We observe that the majority of permanent clogs happen in the first constriction. Li et al. [11] measure the critical pressure at which microgels are forced through a constriction. They find that after going through a first constriction, a smaller critical pressure is necessary for the microgels to go through next constrictions if the particles do not reswell fast enough to their original shape and size. This would imply that once a particle passes the first constriction, it would pass all of them, but that is typically not what we find since clogging deep in the pores is also observed. This in turn indicates that most probably the microgels we use are able to reswell fast. The critical pressure can also help us explain the change in system selectivity with pressure.

We also found that the change in shape of the microgels does depend on the channel entrance angle, whereas the variation in volume is not dependent on the channel entrance angle since the data is very scattered (see Appendix C). The same observation was made by Li et al. [10]. On small microgels the entrance angle does not have an influence on the shape of the microgels. This happens because when the microgels are inside the constrictions, they are not in contact with the entrance of the channel but only with the internal dimensions of the constriction. For this reason, only the internal dimensions of the constriction will influence the deformation and deswelling of microgels. When the microgel is large, part of it will be inside the constriction and part outside. The part that enters the constriction loses volume and the rest of the microgel keeps their original properties. As soon as the part that was squeezed comes out of the constriction, it reswells. It is then the turn of the part that was still outside of the constriction to deswell. In the end a constant change in volume is observed.

These results are expected to be of importance for membrane filtration of soft particles where particle transmission through the pores can be observed, and is not 
always desired. Pan et al. [9] synthesized fluorescently labelled microgels, and found microgels in the permeate when using microporous membranes with pores much smaller than the diameter of the particles. Understanding this transmission mechanism (also in relation to the pore size distribution) is important for process optimization when the presence of particles in the permeate is not desirable, or where a specific size separation is needed. Other example of areas that can benefit from our results are chromatography, injectable microgels for tissue engineering [20] and studies on blood cell flow [21].

\section{Conclusions}

In this work, we used microfluidic channels to observe the behavior of microgels going through constrictions. We find that most microgels clog the channels at the first channel constriction independently of constriction entrance angle, particle size and applied pressure. We do see a shift in size distribution of the particles that clog the pores with increasing pressure showing a change in selectivity with system parameters such as applied pressure.

Considering the variations in microgel shape and volume, we find that the degree of deformation increases with particle size and is dependent on constriction entrance angle, whereas changes in volume are independent of entrance angle.

These findings are of great importance for various fields such as membrane filtration. They show how soft particles are able to change their conformation under pressure and emphasize the importance of careful analysis of the process as a whole (so including particle deformation) to avoid undesired contamination in the final product. 


\section{Appendix C. Supplementary data}

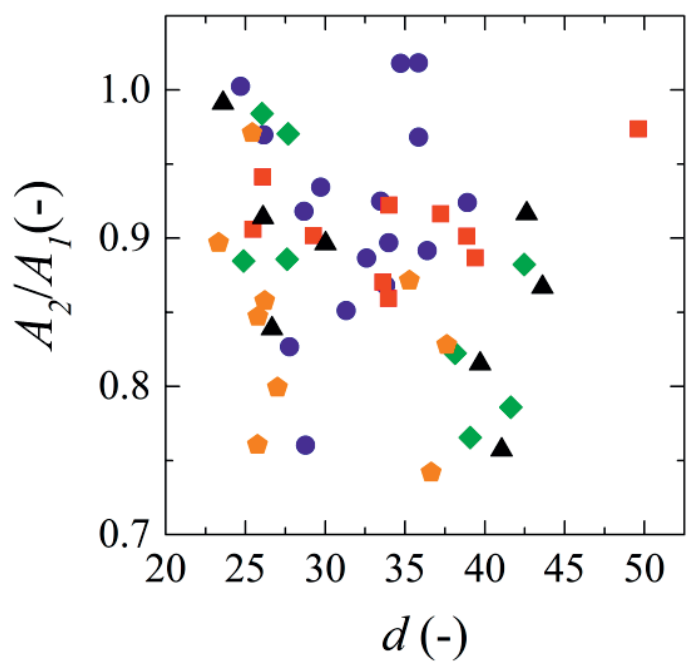

Figure 8. Comparison between deswelling $\left(A_{2} / A_{1}\right)$ and particle size for constriction angles of: Blue circles $0^{\circ}$, red squares $20^{\circ}$, black triangles $35^{\circ}$, green diamonds $45^{\circ}$ and orange pentagons $55^{\circ}$.

\section{Particle compression}

When plotting the area ratio as function of particle size for different entrance angles, we see that microgel deswelling can be as high as $25 \%$, but it is not a function of the particle size or the constriction entrance angle, since the data is very scattered. Small particles may lose up to $25 \%$ area, but this is not that much reflected in the circumference, and therefore this parameter can still be close to 1 . Also, at high pressure the particles will have less time to deswell and thus need to deform more in order to pass the constriction, or vice versa, and this could be an explanation for range of area ratios that are found. 


\section{References}

[1] D.F. Do Nascimento, J. A. Avendaño, A. Mehl, M. J. B. Moura, M. S. Carvalho, W. J. Duncanson, Flow of tunable elastic microcapsules through constrictions, Sci. Rep. 7 (2017) 1-7.

[2] X. Hong, M. Kohne, M. Morrell, H. Wang, E.R. Weeks, Clogging of soft particles in two-dimensional hoppers, Phys. Rev. E 96 (2017) 1-10.

[3] L. Chen, K.X. Wang, P.S. Doyle, Effect of internal architecture on microgel deformation in microfluidic constrictions, Soft Matter 13 (2017) 1920-1928.

[4] K.L. Tung, C.C. Hu, C.J. Chuang, K.J. Hwang, T.Y. Wu, Effects of soft particle deformability and particle/pore size ratio on the blocking mechanism in deadend microfiltration, Chem. Eng. Technol. 33 (2010) 1341-1348.

[5] O. Nir, T. Trieu, S. Bannwarth, M. Wessling, Microfiltration of deformable microgels, Soft Matter 12 (2016) 6512-6517.

[6] E. Dressaire, A. Sauret, Clogging of microfluidic systems, Soft Matter 13 (2017) 37-48.

[7] O. Liot, A. Singh, P. Ba, P. Duru, J.F. Morris, P. Joseph, Pore cross-talk in colloidal filtration, Sci. Rep. 8 (2018) 1-7.

[8] R. van Zwieten, T. van de Laar, J. Sprakel, K. Schroën, From cooperative to uncorrelated clogging in cross-flow microfluidic membranes, Sci. Rep. 8 (2018) 5687.

[9] S. Pan, J. M.G. Tzoc Torres, T. Hoare, R. Ghosh, Transmission behavior of pNIPAM microgel particles through porous membranes, J. Membr. Sci. 479 (2015) 141-147.

[10] Y. Li, O.S. Sarlyer, A. Ramachandran, S. Panyukov, M. Rubinstein, E. Kumacheva, Universal behavior of hydrogels confined to narrow capillaries, Sci. Rep. 5 (2015) 1-11.

[11] Y. Li, E. Kumacheva, A. Ramachandran, The motion of a microgel in an axisymmetric constriction with a tapered entrance, Soft Matter 9 (2013) 1039110403.

[12] E. Benet, A. Badran, J. Pellegrino, F. Vernerey, The porous media's effect on the permeation of elastic (soft) particles, J. Membr. Sci. 535 (2017) 10-19.

[13] A.M.C. Van Dinther, C.G.P.H. Schroën, R.M. Boom, Separation process for very concentrated emulsions and suspensions in the food industry, Innov. Food Sci. Emerg. Technol. 18 (2013) 177-182.

[14] K.J. Hwang, Y.C. Chiang, Comparisons of membrane fouling and separation efficiency in protein/polysaccharide cross-flow microfiltration using membranes with different morphologies, Sep. Purif. Technol. 125 (2014) 7482. 
[15] K.J. Hwang, P.Y. Su, E. Iritani, N. Katagiri, Compression and filtration characteristics of yeast-immobilized beads prepared using different calcium concentrations, Sep. Sci. Technol. 51 (2016) 1947-1953.

[16] I. Bouhid de Aguiar, T. van de Laar, M. Meireles, A. Bouchoux, J. Sprakel, K. Schroën, Deswelling and deformation of microgels in concentrated packings, Sci. Rep. 7 (2017) 10223.

[17] J. Linkhorst, T. Beckmann, D. Go, A.J.C. Kuehne, M. Wessling, Microfluidic colloid filtration, Sci. Rep. 6 (2016) 22376.

[18] N. Debnath and M. Sadrzadeh, Microfluidic mimic for colloid membrane filtration: review, J. Indian Inst. Sci. 98 (2018) 137-157.

[19] T. van de Laar, S. ten Klooster, K. Schroën, J. Sprakel, Transition-state theory predicts clogging at the microscale, Sci. Rep. 6 (2016) 28450.

[20] Y. Hou, W. Xie, K. Achazi, J.L Cuellar-Camacho, M.F. Melzig, W. Chen, R. Haag, Injectable degradable PVA microgels prepared by microfluidic technology for controlled osteogenic differentiation of mesenchymal stem cells, Acta Biomater. 77 (2018) 28-37.

[21] L.I. Amar, D. Guisado, M. Faria, J.P. Jones, C.J.M. van Rijn, M.I. Hill, E.F. Leonard, Erythrocyte fouling on micro-engineered membranes, Biomed. Microdevices 20 (2018) 1-11. 



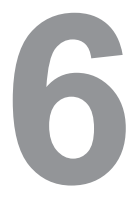

Discussion 


\section{Introduction}

As mentioned throughout this thesis, soft particles behave rather differently from their solid counterparts. The special characteristics of soft particles make it difficult to predict their behavior during separation processes such as filtration [1,2]. From earlier chapters, it is clear that deformability leads for example to the passage of particles through pores that are considerably smaller in size than the diameter of particles; an effect that is not covered by current filtration theory. In the following section, the main findings of each chapter will be highlighted first, and related briefly to practical processes. Finally, the implications of these findings will be put into a wider perspective.

\section{Main findings}

In Chapter 2, we use packings of micrometer-sized gels to investigate the behavior of microgels under external pressure by using the osmotic stress technique. We obtained information on the resistance of the packings to compression and observed the structure of the packings. We found that suspensions of dextran microgels start to resist compression at volume fractions close to random close packing of hard spheres with the same size distribution. For volume fractions between random close packing and 1 , the resistance increases similarly to that of a dextran solution of the same concentration. From image analysis followed that microgels are deformed but internal concentration remains the same. At volume fractions 'higher than 1', microgels are forced to expel solvent and deswell.

The results of Chapter 2 are of importance for processes in which soft particles are exposed to an external force, and pushed into each other as is the case during filtration and centrifugation, or even processing in general where particles are exposed to external stresses. The osmotic stress technique is thus useful to assess properties of the packings [3,4]. In our study, we used a microgel suspension with a broad size distribution which allows particle rearrangement during compression, so smaller particles may be forced to change positions in the interstitial spaces of the packing [5].

When translating this to the situation during membrane filtration in which particles accumulate on top of a membrane, we can relate our findings to the cake resistance that builds up during filtration. Although we cannot do this in a quantitative manner yet, it is clear that our findings can be used to predict the resistance of the layer as function of the applied pressure for example, which in a next step can be linked to the local selectivity of the membrane process, as is further elaborated in Chapter 3. 
This is an important step toward more detailed mechanistic understanding of filtration behavior.

We were intrigued by the observations that microgels of the size that we used would first deform at lower pressure and deswell at higher pressures. For that reason, we decided to carry out a more in-depth analysis of microgel deformation and deswelling. In Chapter 3 we investigated the deformation and deswelling behavior of individual microgels in microgel packings at varying osmotic pressures. We found that in most cases, both deswelling and deformation occur simultaneously; the degree to which each modification occurs depends on the osmotic pressure of the system. Faceting tends to be the dominant modification mechanism at lower pressures while deswelling is more pronounced at higher pressures (Figure 1).

Under these conditions, microgels regain their spherical shape if the pressure of the system continues to increase, although obviously their size becomes smaller. In this chapter, we confirm the observations of Chapter 2 that microgels first deform and afterwards deswell with increasing pressure. We also propose a theory capable of predicting the behavior of microgels according to the applied pressure to the system. This theory and the observations in general can be applied to processes where the structure of packings of soft particles is critical such as chromatography.
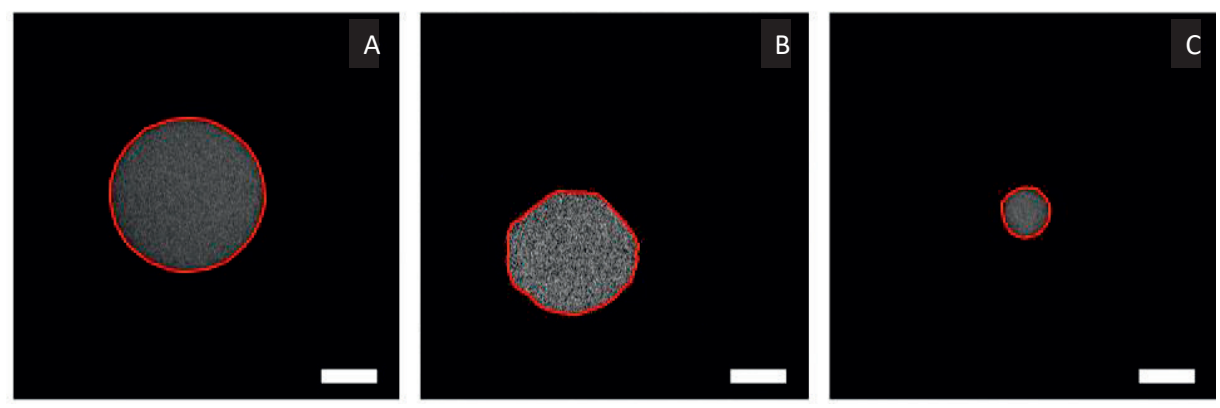

Figure 1. Confocal images of a single microgel at (A) zero pressure, (B) at $\mathrm{P}=10 \mathrm{kPa}$ and (C) $2 \mathrm{MPa}$. Red outlines correspond to the edges of the particle after image analysis. Scale bars denote $5 \mu \mathrm{m}$. Adapted from Chapter 3 .

Membrane cake formation and filtration in general are subjects that can benefit from the results presented in this thesis. With our theory from Chapter 3, it is possible to predict the shape of the microgels depending on applied pressure, and this is a direct link to the retention of particles. As a matter of fact, this could be a way to predict the actual pore size needed to retain particles that are quite smaller than when 
measured away from the applied process conditions. Also, the liquid flux will be influenced by the shape of the soft particles in the membrane cake/deposit layer. When soft particles are deformed, the contact area between them is larger. This means that the space in between the particles will be smaller, therewith decreasing the porosity of this cake/deposit leading to higher resistance against flow. As soon as the particles are forced further together and regain their spherical shape, it is expected that the porosity of the cake/deposit will consequently increase, therewith increasing the liquid flux, and possibly even leading to passage of these small particles through a pore (see also later chapters).

After studying the behavior of microgels in isotropic and static conditions with osmotic stress experiments in the first two experimental chapters, we moved on to using the microgels in dynamic systems that move our observations in model processes closer to those needed to understand filtration (and centrifugation) processes in practice.

In Chapter 4, we use soft particles in microfluidic filtration and centrifugation devices to emulate processes in which they are being subjected to external forces. We found that the propensity of a microgel particle to clog a pore is dependent on the applied pressure. At low pressures, microgels are more likely to clog a pore. As pressure increases, microgels are more likely to be pushed all the way through the pores or block deeper in the pore. We also found that a microgel deposit layer (cake layer) that formed on top of the model membrane can be compressed up to $30 \%$ but the compression is totally reversible, which was also observed in centrifugation trials. Reversibility in centrifugation trials was observed as long as the chip configuration allowed for water to be in close contact with the deposited layer. In the absence of water, the layer compressed irreversibly. The conclusion is that microgel deposit compression can be totally reversed as long as there is water available for reswelling.

Our observations on the behavior of soft particle deposits shed new light on behavior of cake layers in membrane filtration, and even more precisely on the management of deposit formation, which is one of the challenges faced during membrane filtration. The overall management of fouling as this effect is known in the membrane field, is mostly solved through the application of high shear in combination with extensive cleaning [6,7]. Our results clearly indicate that the pressure drop applied during filtration may lead to tighter cake layers through which less liquid will permeate, and relatively small particles will be captured. When thinking in terms of product quality, this will influence both productivity and selectivity of the process greatly. Likewise, it will also influence the efficiency of any high shear cleaning step 
that is carried out to mitigate layer formation [8]. The critical pressure above which gel reversibility is no longer observed, is normally not considered in regular process design and cleaning operation. We have shown that this critical pressure also depends on particle properties, but we only briefly touched upon this effect that is worthy of a deeper study.

In Chapter 4, we investigated relatively small particles collectively going through pores. In Chapter 5, we zoom in and observe individual particles going through pores/constrictions as in a model membrane system. We see that most microgels clog at the first channel constriction independently of the constriction entrance angle and applied pressure. Higher pressures promote clogging deeper in the channels but most of the microgels will still clog at the first constriction. This was a remarkable finding, since this also indicates that cross-flow as standardly used will not be effective to mitigate this effect. We also observe a shift in particle size of microgels that clog the pores with increasing applied pressure, as could be expected from previous chapters: particles decrease their size with increasing pressure and are more likely to go through a pore. The degree of particle deformation is dependent on channel entrance angle whereas changes in volume are not. From this it is clear that system selectivity is influenced by the process parameters and the pore geometry, therewith indicating again that choosing a membrane pore size based on the particle diameter under non-process conditions will lead to 'unexpected' filtration behavior that can now much better be understood through our observations.

The main message of this chapter is that soft particles are able to modify their conformation considerably, and thus they can be pushed all the way through pores that are smaller than the particle diameter. Whether a particle gets trapped will depend on process parameters such as applied pressure as well as particle properties. The fact that soft particles that can be up to two times the constriction size can be pushed through the pores show that this capacity of the particles should be taken into consideration for membrane selection to avoid process failure. It also shows that this complexity needs to be taken into account.

\section{Next steps and further interpretation}

In our research, we used a microfluidic system as a model membrane for our research. The model membrane used was composed of non-connecting straight through channels. The channels also had a fixed amount of constrictions, same pore size and five different entrance angles. It was in this highly ideal system that we obtained the results presented in the last two chapters of this thesis. However, real membranes differ from this ideal system in pore configuration, pore size distribution 
and pore design. These topics will be discussed in the following paragraphs together with some suggestions on the use of model particles and model suspensions.

\section{Pore configuration}

In general, membranes have a variety of pore configurations: straight-through, interconnected and even dead-end pores [9]. This means that soft particles can feel the applied pressure in different ways according to their position inside the membrane. When soft particles are under pressure, it is like that they will deswell/deform according to particle characteristics and applied pressure. For this reason, soft particles going through pores with different configurations can experience different amounts of pressure and consequently deswell in different ways changing their water content and softness. All these changes are expected to occur in real membrane configurations and were not taken into consideration in this thesis. From the observation that plugging often occurs near the first constriction (Chapters 4 and 5) in model membranes, also follows that this could be a very important effect in practice.

\section{Pore size distribution}

Another particularity of our model system is that all channel/pore entrances had the same size and that is not true for most membranes. Most membranes used on large scale do not have pores with the exact same size but a pore size distribution [10]. This is mainly due to the fact that producing membranes with identical pore sizes is challenging and expensive [11,12]. For a membrane with a pore size distribution, it is expected that the largest pores carry a bigger portion of the permeate, and thus the separation characteristics of the overall process would depend greatly on the size of these pores: both flux and retention would be affected by this. The initial retention would be determined by the ratio of particle over pore size for the process conditions used (see earlier remarks on particle size to be used in practice) [13], but when these pores get blocked the next largest pores carry more liquid and retention is shifted to smaller particles. We expect pore size distribution to have an influence on the deswelling/deformation behavior of soft particles and consequently on filtration behavior in general. In our research, we used particles with a broad size distribution and larger and smaller particles were added to the system simultaneously. What we observed is that smaller particles easily passed through the pores while larger particles were responsible for clogging the pores/constrictions. After clogging, smaller particles were also retained to form a cake layer. In a system with a pore size distribution, smaller particles can thus be expected to be responsible for pore clogging possibly together with larger ones and that would change clogging dynamics. 


\section{Pore design}

Our model membrane has five different pore entrance designs. In a real membrane, there would be no designs, but many pores coexisting (pore matrix) with various entrance angles unless they are isoporous membranes [11,12]. Although we covered a range of entrance angles in our research, alternative entrance angles and designs could also be tested in a similar way.

\section{Model particles}

In this thesis, we used model particles in suspension. The suspensions were ideal since they contained soft particles in the micrometer range, what excluded colloidal interactions that may play a role in practical systems $[14,15]$. In Chapter 2, we used microgels with different properties such as size distribution, whereas in the following chapters, a single type of microgel suspension was used. It would be insightful to investigate the effect of particle properties such as softness and size distribution on filtration behavior in the systems studied. As a next step, various types of particles, with different compositions and size distributions can be combined, to emulate filtration in practice more closely. Also, interactions between particles would be interesting to investigate in the model membrane systems, since it is expected that this can greatly influence filterability of a feed solution as described by Van de Laar et al. [16] for hard particles.

\section{Model suspensions}

When going from the rather ideal liquids used here, and considering non-ideal liquids, the implications for concentration and separation processes can also differ. When concentrating ideal microgel suspensions, only the suspension as a whole will be concentrated. In non-ideal mixtures, depending on the selectivity of the membrane, the concentration of not only particles, but also solutes can completely change the behavior of the retentate. When the final objective of the membrane process is separation, it is easier to predict the behavior of ideal mixtures, but when fractionation takes place this is much more complex [17]. Furthermore, the presence of different types of particles and solutes can promote particle interactions and also influence/create interactions between the particles which may lead to shear-induced diffusion that is known to improve membrane processes. When the interactions are between particles and the membrane, this is mostly detrimental for filtration [6,18]. These considerations clearly indicate that doing extended research with non-ideal liquids can bring extra information needed to design filtration processes. In short, every filtration process is unique, and for that reason, the more information available to help with process design, the better, and for this model membranes as used in this work are of great value. 


\section{Extra observations}

During our in-situ microscopy observation experiments, we saw many peculiarities that could be subject of follow-up research. Among these topics are particle adhesion, the movement of the smaller particles through the packing and kinetics of reswelling and deformation. We observed that when a clog would be released, the particles accumulated on top of it would be released in chunks and not immediately as individual particles (Figure 2). We also observed that when a constriction is clogged and there is accumulation of particles on top of it, small particles still move in between the bigger particles and ultimately move through the pore. Studying the behavior of these particles could bring more information on the structure of the accumulation layer itself.
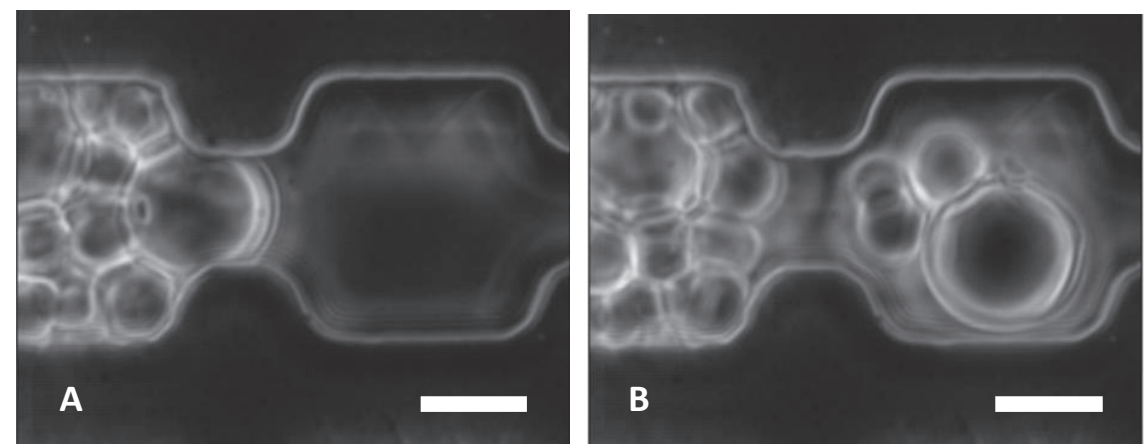

Figure 2. Microgel in constriction A) before release and B) after release showing agglomeration. Scale bar denotes $20 \mu \mathrm{m}$.

Finally, we observed that once a microgel goes through one constriction, it is more likely that it will go through more constrictions at the same shot. This can be related to the reswelling kinetics, since the particle may not have had enough time to reswell to the size in which it initially was caught in the first constriction. We did not quantify/study this behavior, but it surely would help understanding of soft particle filtration to a next level. For straight through pores (with constrictions) this would imply that if a particle can move through the first constriction, it will move all the way through the pores if it does not reswell fast, which would also greatly help the filtration process as a whole. The monitoring and understanding of the kinetics of particle deswelling and reswelling could help the explanation of this observation and bring more information on particle behavior in general.

Throughout this thesis, we used two-dimensional observations to investigate the systems. However, three-dimensional observations could bring a more precise 
insight into the behavior of individual particles in the system as a whole and closer to real situations and applications. Alternative microscopy techniques could be used for this purpose such as three-dimensional reconstruction of confocal images. The observation of microgel packings/cake layers/deposits in a three-dimensional way can bring more details on structure as for example the number and size of contact points and the position of smaller particles before and after rearrangements.

\section{Concluding remarks}

In our research and discussion, we focused on membrane filtration processes, and showed how the model micro systems can be used to generate mechanistic understanding. However, our results are also relevant for other fields of application, mainly those using packings of soft particles. One example of such application is chromatography. In our first experimental chapter (Chapter 2), we use Sephadex microgels as model particles, that were developed to be used in chromatography columns, and our results immediately link to chromatography processes.

All the differences between our model membrane system and real applications mentioned in the previous sections should be subject of extended research since such topics are only touched upon in this work. However, it is clear that the tools used in this work and the methodology developed can be applied very widely to different fields of application and systems. 


\section{References}

[1] A. Bouchoux, P. Qu, P. Bacchin, G. Gésan-Guiziou, A general approach for predicting the filtration of soft and permeable colloids: the milk example, Langmuir 30 (2014) 22-34.

[2] M. Elimelech, S. Bhattacharjee, A novel approach for modeling concentration polarization in crossflow membrane filtration based on the equivalence of osmotic pressure model and filtration theory, J. Membr. Sci. 145 (1998) 223241.

[3] C. Bonnet-Gonnet, L. Belloni, B. Cabane, Osmotic pressure of latex dispersion, Langmuir 10 (1994) 4012-4021.

[4] M. Rubinstein, R.H. Colby, A.V. Dobrynin, Dynamics of semidilute polyelectrolyte solutions, Phys. Rev. Lett. 73 (1994) 2776-2779.

[5] S. Nezamabadi, T.H. Nguyen, J.-Y. Delenne, F. Radjai, Modeling soft granular materials, Granul. Matter 19 (2017) 8.

[6] L. E. Head, M. R. Bird, Backwashing of tubular ceramic microfilters fouled with milk protein isolate feeds, J. Food Process Eng. 36 (2013) 228-240.

[7] A. Ostadfar, A. H. Rawicz, M. Gitimoghaddam, Application of backwashing to increase permeate flux in bioparticle separation, J. Med. Biol. Eng. 33 (2013) 478-485.

[8] L. Böhm, A. Drews, H. Prieske, P.R. Bérubé, M. Kraume, The importance of fluid dynamics for MBR fouling mitigation, Bioresource Technol. 122 (2012) 50-61.

[9] M.A. Islam, M. Ulbricht, Microfiltration membrane characterization by gas-1 iquid displacement porometry: Matching experimental pore number distribution with liquid permeability and bulk porosity, J. Memb. Sci. 569 (2019) 104-116.

[10] J. Woods, J. Pellegrino, J. Burch, Generalized guidance for considering poresize distribution in membrane distillation, J. Memb. Sci. 368 (2011) 124-133.

[11] V. Abetz, Isoporous block copolymer membranes, Macromol. Rapid Commun. 36 (2015) 10-22.

[12] S. Rangou, K. Buhr, V. Filiz, J.I. Clodt, B. Lademann, J. Hahn, A. Jung, V. Abetz, Self-organized isoporous membranes with tailored pore sizes, J. Memb. Sci. 451 (2014) 266-275.

[13] R. Baker, Membrane Technology and Applications, 2nd ed., John Wiley \& Sons, Ltd, Chichester, 2004.

[14] K. L. Tung, C. C. Hu, C. J. Chuang, K. J. Hwang, T. Y. Wu, Effects of soft particle deformability and particle/pore size ratio on the blocking mechanism in dead-end microfiltration, Chem. Eng. Technol. 33 (2010) 1341-1348. 
[15] O. Nir, T. Trieu, S. Bannwarth, M. Wessling, Microfiltration of deformable microgels, Soft Matter 12 (2016) 6512-6517.

[16] T. van de Laar, S. ten Klooster, K. Schroën, J. Sprakel, Transition-state theory predicts clogging at the microscale, Sci. Rep. 6 (2016) 28450.

[17] A.M.C. van Dinther, C.G.P.H. Schroën, R.M. Boom, Particle migration leads to deposition-free fractionation, J. Memb. Sci. 440 (2013) 58-66.

[18] H. P. Chu, X.-Y. Li, Membrane fouling in a membrane bioreactor (MBR): sludge cake formation and fouling characteristics, Biotechnol. Bioeng. 90 (2005) 323-331. 


\section{Summary}

Soft particles are present in our daily lives and differently from their hard counterparts, they can change conformation and composition when experience an external source of stress. This specific characteristic of soft particles can make it more challenging to predict their behavior in processes such as filtration and centrifugation. More information on the specific behavior of soft particles under external stress is still lacking on current literature and can be useful to different areas of application.

The aim of this thesis is to provide information that will contribute to the understanding of soft particle behavior under pressure such as in pore clogging and cake formation in membrane processes. We use micrometer-sized microgels as model particles in this work due to their tunability and ease of production. Also, using micrometer-sized microgels we can consider colloidal interactions negligible, what simplifies our system and allows us to focus on individual particle behavior.

In the first two experimental chapters (Chapters 2 and 3), we focus on microgel packings (static conditions). The packings were produced by osmotic stress with controlled, varying applied pressure.

In Chapter 2, we focus on the collective behavior of microgels in packings in static conditions and we describe the behavior of the microgel packings in term of wellknown polymeric theories such as the Flory-Rhener theory. We found that suspensions of dextran microgels start to resist compression at volume fractions close to random close packing of hard spheres with the same size distribution. For volume fractions between random close packing and 1, the resistance increases similarly to that of a dextran solution of the same concentration. From image analysis followed that microgels are deformed but internal concentration remains the same. At volume fractions 'higher than 1', microgels are forced to expel solvent and deswell.

In Chapter 3, we explore our observation from Chapter 2 that individual particles will respond to stress in different ways according to the applied pressure. For that we use microgel packings containing a mixture of fluorescent and non-fluorescent microgels with an excess of non-fluorescent microgels. We observe the packing using fluorescence microscopy and are able to observe single fluorescent particles surrounded by non-fluorescent particles (non-visible). We found that both deswelling and deformation occur simultaneously when soft particles are under pressure and we describe a theory to predict their behavior according to the pressure applied to the system. 
In Chapters 4 and 5, we use microfluidic devices to observe the behavior of soft particles in dynamic systems.

In Chapter 4, we focus on the collective behavior of particles. For that we use a microcentrifuge coupled with an optical microscope to investigate the reversibility of soft particle deposits according to the applied force. We found that, for the particles used, total reversibility of deposits is possible as long as there is water available for particle reswelling. Also in Chapter 4, we use microfluidic devices composed of an array of parallel channels as a model membrane for filtration experiments. In this device, we observe the clogging behavior of soft particles in filtration, focus on cake layer formation and assess cake reversibility. We found that the propensity of a particle to clog is dependent on the applied pressure and that at low pressures, microgels are more likely to clog a pore. As pressure increases, microgels are more likely to be pushed all the way through the pores or block deeper in the pore. We also found that a microgel deposit layer (cake layer) that formed on top of the model membrane can be compressed up to $30 \%$ but the compression is totally reversible.

After focusing on collective behavior of soft particles, in Chapter 5 we focus on what is happening at individual particle level. We observe single particles going through pore constrictions and assess deformation and deswelling of the particles. We then correlate the observations with particle and system properties such as particle size and applied pressure. We found that higher pressures promote clogging deeper in the channels but most of the microgels will still clog at the first constriction. We also observe a shift in particle size of microgels that clog the pores with increasing applied pressure, as could be expected from previous chapters: particles decrease their size with increasing pressure and are more likely to pass a pore. The degree of particle deformation is dependent on channel entrance angle whereas changes in volume are not.

Finally, in Chapter 6, we discuss our main findings and their implications in real life situations and processes. The results presented in this thesis are of importance in many areas involving packings and concentration of soft particles such as membrane filtration and chromatography. 


\section{Résumé}

Les particules molles sont présentes dans notre vie quotidienne et, contrairement à leurs homologues durs, elles peuvent changer de conformation et de composition lorsqu'elles sont confrontées à une source de stress externe. Cette caractéristique spécifique des particules molles peut rendre plus difficile la prévision de leur comportement dans des processus tels que la filtration et la centrifugation. De plus amples informations sur le comportement spécifique des particules molles sous contrainte externe font encore défaut dans la littérature actuelle et peuvent être utiles dans différents domaines d'application.

Le but de cette thèse est de fournir des informations qui contribueront à la compréhension du comportement des particules molles sous pression, telles que le colmatage des pores et la formation de gâteaux dans les procédés de filtration membranaires. Nous utilisons des microgels de taille micrométrique comme particules modèles en raison de leur adaptabilité et de leur facilité de production. De plus, en utilisant des microgels de taille micrométrique, nous pouvons considérer les interactions colloïdales comme négligeables, ce qui simplifie notre système et nous permet de nous concentrer sur le comportement des particules individuelles.

Dans les deux premiers chapitres expérimentaux (chapitres 2 et 3), nous nous concentrons sur des empilements de microgels obtenus en conditions statiques. Ces empilements ont été produits par stress osmotique dans une gamme de pression contrôlée.

Au chapitre 2, nous nous concentrons sur le comportement collectif des microgels dans des empilements statiques et décrivons leur comportement, notamment leur résistance à la compression et à la déformation dans le cadre de la théorie de FloryRhener. Nous avons constaté que les suspensions de microgels de dextrane commençaient à résister à la compression pour des fractions volumiques proches de la compacité pour un empilement aléatoire de sphères dures présentant la même distribution de taille. Aux fractions volumiques comprises entre la compacité d'un empilement compact aléatoire de sphères et 1, la résistance augmente de zéro à des valeurs proches de celle d'une solution de dextran de même concentration. L'analyse d'images obtenues par microscopie confocale des empilements a ensuite montré que les microgels sont déformés mais que la concentration interne reste la même. Aux fractions volumiques supérieures à 1 , les microgels sont obligés d'expulser le solvant et de se dégonfler, ce qui réduit leur taille. 
Au chapitre 3, nous examinons l'observation tirée du chapitre 2 selon laquelle les particules individuelles réagiront au stress de différentes manières en fonction de la pression appliquée. Pour cela, nous utilisons des empilements de microgels contenant un mélange de microgels fluorescents et non fluorescents avec un excès de microgels non fluorescents. Nous observons la compaction à l'aide de la microscopie à fluorescence et pouvons observer des particules fluorescentes simples entourées de particules non fluorescentes (non visibles). Nous avons constaté que la réduction de taille et la déformation se produisent simultanément lorsque les particules molles sont sous pression et nous décrivons une théorie permettant de prédire leur comportement en fonction de la pression appliquée au système.

Dans les chapitres 4 et 5 , nous utilisons des dispositifs microfluidiques pour observer le comportement des particules molles dans des systèmes dynamiques.

Au chapitre 4, nous nous intéressons au comportement collectif des particules. Pour cela, nous utilisons une microcentrifugeuse couplée à un microscope optique pour étudier la réversibilité des dépôts de particules molles en fonction de la force appliquée. Nous avons constaté que, pour les microgels utilisés, une réversibilité totale des dépôts après compaction est possible dans la mesure où il existe de l'eau disponible pour le regonflement des microgels Toujours au chapitre 4, nous utilisons des dispositifs microfluidiques composés d'un ensemble de canaux parallèles comme membrane modèle pour les expériences de filtration. Dans ce dispositif, nous observons le comportement colmatant des particules molles lors de la filtration. Nous nous concentrons sur la formation de la couche de gâteau et évaluons la réversibilité du gâteau. Nous avons constaté que la propension d'un microgel à boucher un canal dépend de la pression appliquée et qu'à des pressions basses, les microgels sont plus susceptibles de boucher un canal. À mesure que la pression augmente, les microgels sont plus susceptibles d'être poussés à travers les pores ou de se bloquer plus profondément dans les pores. Nous avons également constaté qu'une couche de dépôt de microgel (couche de gâteau) qui se formait au-dessus de la membrane modèle pouvait être comprimée jusqu’à 30\%, mais que la compression était totalement réversible.

Après avoir abordé le comportement collectif des particules molles, au chapitre 5 , nous nous concentrons sur ce qui se passe au niveau des particules individuelles. Nous observons des particules individuelles traversant des constrictions de pores et évaluons leur déformation et leur dégonflement Nous établissons ensuite une corrélation entre les observations et les propriétés des particules et du système, telles que la taille des particules et la pression appliquée. Nous avons constaté que des 
pressions plus élevées favorisaient un encrassement plus profond dans les canaux, mais que la plupart des microgels resteraient encrassés à la première constriction. Nous observons également un changement dans la taille des particules de microgels qui obstruent les pores lorsque la pression appliquée augmente, comme on pouvait s'y attendre au vu des résultats des chapitres précédents: les particules diminuent de taille avec l'augmentation de la pression et ont plus de chances de traverser un pore. Le degré de déformation des particules dépend de l'angle d'entrée du canal alors que les changements de volume n'en dépendent pas.

Enfin, au chapitre 6, nous discutons de nos principales conclusions et de leurs implications dans des situations et processus réels. Les résultats présentés dans cette thèse sont importants dans de nombreux procédés séparatifs impliquant la concentration de particules molles, tels que les procédés de filtration notamment membranaire, la centrifugation mais aussi la chromatographie. 


\section{Samenvatting}

Zachte deeltjes zijn overal in ons dagelijks leven aanwezig, en in tegenstelling tot harde deeltjes kunnen ze van vorm en samenstelling veranderen wanneer ze een externe bron van druk ervaren. Deze specifieke eigenschap van zachte deeltjes maakt het uitdagend om hun gedrag te voorspellen in processen zoals filtratie en centrifugatie. Kennis en informatie over het specifieke gedrag van zachte deeltjes onder externe druk ontbreekt momenteel nog in de literatuur, terwijl dit nuttig zijn voor verschillende toepassingsgebieden.

Het doel van dit proefschrift is om informatie te verschaffen die zal bijdragen aan het begrip van het gedrag van zachte deeltjes onder druk, zoals bij verstopping van poriën en vorming van koeklagen tijdens membraanprocessen. We gebruiken microgels van micrometer grootte als modeldeeltjes, vanwege de mogelijkheden om hun eigenschappen aan te passen, en het gemak van productie. Ook kunnen we hierdoor colloïdale interacties verwaarlozen, wat ons systeem vereenvoudigt en ons in staat stelt om ons te concentreren op individueel deeltjesgedrag.

In de eerste twee experimentele hoofdstukken (hoofdstukken 2 en 3), richten we ons op zogenaamde microgel-pakkingen (onder statische omstandigheden). Deze pakkingen werden gemaakt door een gecontroleerde variabele osmotische stress toe te passen.

In hoofdstuk 2 concentreren we ons op het collectieve gedrag van microgels in pakkingen onder statische condities en beschrijven we het gedrag van de pakkingen in termen van bekende polymeerconcepten, zoals de Flory-Rhener-theorie. We ontdekten dat suspensies van dextran-microgels bestand zijn tegen compressie bij volumefracties in de buurt van een dichtste bolstapeling van harde bollen met dezelfde grootteverdeling. Voor volumefracties tussen de dichtste bolstapeling en 1, neemt de weerstand toe op dezelfde manier als in een dextran-oplossing met dezelfde concentratie; de microgels vervormen maar de interne concentratie blijft hetzelfde. Bij volumefracties 'hoger dan 1', worden microgels gedwongen om oplosmiddel uit te stoten en krimpen.

In hoofdstuk 3 gaan we verder op onze observatie uit hoofdstuk 2 dat individuele deeltjes op verschillende manieren op druk zullen reageren, afhankelijk van de toegepaste druk. Daarvoor gebruiken we microgel-pakkingen die een mengsel van fluorescerende en niet-fluorescerende deeltjes bevatten met een overmaat aan nietfluorescerende microgelen. We observeerden de pakking met behulp van 
fluorescentiemicroscopie en waren in staat om fluorescerende deeltjes te observeren omringd door niet-fluorescerende deeltjes. We ontdekten dat zowel krimp als vervorming gelijktijdig optreden wanneer zachte deeltjes onder druk staan, waarvoor we theorie beschrijven om het gedrag van zachte deeltjes als functie van de druk die op het systeem wordt uitgeoefend.

In hoofdstukken 4 en 5 gebruiken we microfluidische apparaten om het gedrag van zachte deeltjes in dynamische systemen te observeren.

In hoofdstuk 4 concentreerden we ons op het collectieve gedrag van deeltjes. Hiervoor gebruiken we een microcentrifuge gekoppeld aan een optische microscoop om de reversibiliteit van samendrukking van zachte deeltjes te onderzoeken als functie van de uitgeoefende kracht. We vonden dat voor de gebruikte deeltjes, totale omkeerbaarheid mogelijk is zolang water beschikbaar om de deeltjes te laten zwellen. Ook gebruikten we microfluidische apparaten bestaande uit een reeks parallelle kanalen als een modelmembraan voor filtratie-experimenten. In dit apparaat observeerden we het verstoppingsgedrag van zachte deeltjes tijdens filtratie, en focussen we op de vorming van koeklagen en de omkeerbaarheid hiervan. We vonden dat de neiging van een deeltje om een porie te verstoppen afhankelijk is van de toegepaste druk en dat naarmate de druk lage is de kans hoger is dat microgels een porie verstoppen. Bij hogere druk, krijgen microgels meer de kans om helemaal door de poriën geduwd te worden of de porie dieper te blokkeren. We ontdekten ook dat een afzetting van microgelen (koeklaag) bovenop het modelmembraan tot 30\% kan worden gecomprimeerd, en dat deze compressie volledig omkeerbaar is.

$\mathrm{Na}$ ons te hebben gericht op collectief gedrag van zachte deeltjes, richtten we ons in hoofdstuk 5 op wat er op individueel deeltjes-niveau gebeurt. We observeerden individuele deeltjes die door porie-vernauwingen gaan en beoordeelden de vervorming en het krimpen van de deeltjes. De meeste microgels verstopping de kanalen nog steeds bij de eerste vernauwing, hoewel bij hogere druk ook verstopping dieper in het kanaal optreedt. We zagen ook een verschuiving in de grootte van microgelen die de poriën verstoppen bij toenemende uitgeoefende druk, zoals verwacht op basis van de resultaten van de vorige hoofdstukken: deeltjes worden kleiner met toenemende druk en maken meer kans om door een porie te gaan. De mate van deeltjesvervorming is afhankelijk van de kanaalinvoerhoek, terwijl veranderingen in volume dat niet zijn.

Tot slot bespreken we in hoofdstuk 6 onze belangrijkste bevindingen en hun implicaties in reële situaties en processen. De resultaten gepresenteerd in dit 
proefschrift werpen een nieuw licht op veel gebieden waarin met pakkingen en concentratie van zachte deeltjes wordt gewerkt, zoals membraanfiltratie en chromatografie. 


\section{List of publications}

I. Bouhid de Aguiar, T. van de Laar, M. Meireles, A. Bouchoux, J. Sprakel, and K. Schroën, Deswelling and deformation of microgels in concentrated packings, Scientific Reports, vol. 7, no. 1, p 10223, 2017

I. Bouhid de Aguiar, K. Schroën, M. Meireles, A. Bouchoux, Compressive resistance of granular-scale microgels: From loose to dense packing, Colloids and Surfaces A, vol 553, p 406-416, 2018

I. Bouhid de Aguiar, M. Meireles, A. Bouchoux, and K. Schroën, Microfluidic model systems used to emulate processes occurring during soft particle filtration, Scientific Reports, vol. 9, no. 1, p. 3063, 2019.

I. Bouhid de Aguiar, M. Meireles, A. Bouchoux, and K. Schroën, Conformational changes influence clogging behavior of micrometer-sized microgels in idealized multiple constrictions. Submitted. 


\section{Acknowledgements}

On this same day 13 years ago, on June 5th 2006, I set foot for the first time on the university to start my academic education. I remember when I used to think about the full academic journey up to having a $\mathrm{PhD}$. I would count on my fingers how many years it would take and how old I would be when I would complete it. Back then, it sounded so far away and unattainable but here I am now, at the end of the journey. It was not an easy one but totally worth it.

First of all, I would like to thank Martine and Antoine for proposing the research topic and for believing in me for working on this project. Thank you for all the scientific discussions and support.

I also thank Karin for jumping onboard this big challenge that was finishing this $\mathrm{PhD}$ project in Wageningen. Thank you for always believing we would get to the end of it, even in the hardest of times. Your support and encouragement were very important to me.

This $\mathrm{PhD}$ project was part of a joint mobility program and for this reason I was able to work in different universities and research groups and meet (or meet again) wonderful people.

The Université Paul Sabatier was my home university and I was lucky to be able to work on the Laboratoire de Génie Chimique where I shared part of my PhD journey with so many incredible people. From my fellow $\mathrm{PhD}$ candidates, to the technicians and professors. Thank you all for all the support and nice memories. A special thanks to Lucía, for being always there for me. From motivational talks to giving me shelter when my room was flooded with hot boiler water :)

I would like to thank Kitty for accepting me for my first mobility in her group in the University of Twente. It was like coming home. I also thank all the people and employees of the group for making my stay so enjoyable and my work possible.

When moving to Wageningen for my last mobility, I felt very welcome by all the people working on the FPE group. Thank you all for being so nice and helpful, for the lunch walks and many other memories.

I also would like to thank Joris and Ties for the fruitful collaboration we had. It was a pleasure working with you. 
Muito obrigada a toda minha família que desde cedo me ensinou a importância do conhecimento e do aprendizado. Em especial aos meus pais Maria Aparecida e Miguel e ao meu irmão Rafael. Muito obrigada por estarem sempre ao meu lado apesar da distância. Amo muito vocês!

To Erik, thank you for being always there for me during this journey. Thank you for being my best friend, motivator, proof-reader, translator, editor and everything I ever needed you to be. I love you and I would never have done it without you. 


\section{About the Author}

Izabella Bouhid de Aguiar was born in Além Paraíba, Brazil on March 3d, 1988 and grew up in Volta Redonda, Brazil, where she graduated from high school in December 2005. In 2006, she moved to Seropédica, Brazil were she followed a fiveyears Food Engineering program at Universidade Federal Rural do Rio de Janeiro. During her studies she participated in student exchange programs in Japan (United Nations University) and in the USA (University of Maryland). She did her internship at EMBRAPA-CTAA research center in Rio de Janeiro, Brazil where she worked on assessing the sensory and physicochemical properties of apple juice clarified and concentrated using membrane filtration processes. In 2011, Izabella started to follow the Erasmus Mundus Master in Membrane Engineering program. In the first year she took courses in the university of Montpellier 2 in France and in the Institute of chemical technology in Prague, Czech Republic. On the second year of her master program Izabella moved to the Netherlands to take courses in the University of Twente in Enschede where she also did her thesis research on the separation and isolation of amino acids by electrodialysis for biorefinary purposes. She obtained her master diploma in August 2013 and in November 2013, she started her PhD program in the framework of the Erasmus Mundus Doctorate in Membrane Engineering program. During this joint doctorate program. Izabella worked in France, at the University of Toulouse 3 - Paul Sabatier and in the Netherlands, at University of Twente and at Wageningen University. The results of her $\mathrm{PhD}$ work are described in this thesis. 


\section{Overview of completed training activities}

\begin{tabular}{|c|c|c|}
\hline \multicolumn{3}{|l|}{ Discipline specific activities } \\
\hline XXXI EMS Summer School ${ }^{b}$ & Unical & 2014 \\
\hline Joint Workshop - EM3E\&EUDIME & Umontpellier & 2014 \\
\hline European Conference on Fluid Particle Separation ${ }^{a}$ & INP & 2014 \\
\hline CNRS GDR AMC2 & CNRS & $2014 / 2015$ \\
\hline Training period at University of Twente & Utwente & 2015 \\
\hline EUDIME meeting & Unical & 2015 \\
\hline Euromembrane ${ }^{b}$ & RWTH & 2015 \\
\hline $5^{\text {th }}$ International Colloids Conferenceb & Elsevier & 2015 \\
\hline European Colloid \& Interface Society Conferencea & ECIS & 2017 \\
\hline \multicolumn{3}{|l|}{ General courses } \\
\hline Management of the doctoral project & Utoulouse & 2014 \\
\hline Doctoriales (PhD week) & Utoulouse & 2014 \\
\hline Academic research and its hiring process & Utoulouse & 2014 \\
\hline Recruitment Interview & Utoulouse & 2015 \\
\hline Getting started with online collaborative tools & Utoulouse & 2016 \\
\hline Introduction to business culture & Utoulouse & 2016 \\
\hline Get prepared for an international career & Utoulouse & 2016 \\
\hline Career Perspectives & WGS & 2016 \\
\hline Scientific Writing & WGS & 2016 \\
\hline \multicolumn{3}{|l|}{ Optionals } \\
\hline Preparation of research proposal & & 2013 \\
\hline Journée des doctorants du LGC & LGC & $2013 / 2014$ \\
\hline Journée des doctorants MEGeP 2014 & MEGeP & 2014 \\
\hline GIMDays $^{\mathrm{a}}$ & LGC & $2014 / 2015$ \\
\hline Soft Matter colloquia ${ }^{a}$ & UTwente & 2015 \\
\hline Germany Study Tour & UTwente & 2015 \\
\hline PhD trip (Germany and Switzerland) & FPE & 2016 \\
\hline
\end{tabular}


The research described in this thesis was financially supported by the European Commission - Education, Audiovisual and Culture Executive Agency (EACEA) in the framework of the program Erasmus Mundus Doctorate in Membrane Engineering (EUDIME).

Cover design by Dennis Hendriks. Photo by Izabella Bouhid de Aguiar Printed by Digiforce - Proefschriftmaken.nl in 150 copies 

